Check for updates

Cite this: Mater. Adv., 2021, 2, 6839

Received 7th May 2021, Accepted 12th August 2021

DOI: 10.1039/d1ma00418b

rsc.li/materials-advances

\section{Advanced developments in nonstoichiometric tungsten oxides for electrochromic applications}

\author{
Shah Zeb, ${ }^{a b}$ Guoxin Sun, (D) ${ }^{\text {ab }}$ Yong Nie, ${ }^{a}$ Huiyan $X u{ }^{a}$ Yu Cui (D) ${ }^{b}$ and \\ Xuchuan Jiang (D) *a
}

\begin{abstract}
Nanoscale tungsten oxides $\left(\mathrm{WO}_{3} / \mathrm{W}_{18} \mathrm{O}_{49}\right)$ are a hot research topic because of their impeccable performance in different fields, such as multi-step responses to one stimulus or selective response to a stimulus under controlled conditions, particularly electrochromic performance and energy saving applications in dynamic controllable smart windows. To satisfy different environments, the transparency and thermal insulation of windows are particularly tailored to reduce energy consumption, thereby decreasing cooling and heating costs. In this review, the nonstoichiometric tungsten oxides $\left(\mathrm{WO}_{3-x}\right)$ are presented in detail, summarized, and discussed, focusing on various methods for the synthesis of $\mathrm{W}_{18} \mathrm{O}_{49}$ and $\mathrm{WO}_{3}$, such as chemical vapor deposition (CVD), physical vapor deposition (PVD), sol-gel, solvothermal, and hydrothermal methods, together with the synthetic strategies for the corresponding thin coatings. Then, the advanced strategies to improve the EC performance of nonstoichiometric $\mathrm{W}_{18} \mathrm{O}_{49}$ nanostructures for multifunctionality EC smart windows are discussed. Furthermore, the fundamental mechanisms in coloration/ bleaching related to oxygen vacancies, hybridization, elemental doping and surface decoration are explored and discussed in detail. Finally, the key challenges, future developments, and remarks to improve the variability of EC smart windows, such as energy harvesting and energy storing multifunction, coloration enhancement, stability, durability, and reducing toxicity are also highlighted accordingly.
\end{abstract}

\section{Introduction}

The growing energy and global environmental issues require advanced, renewable, and sustainable energy resources, especially energy-efficient functional materials. Smart windows are

\footnotetext{
${ }^{a}$ Institute for Smart Materials \& Engineering, University of Jinan, No. 336 Nanxinzhuang West Road, Jinan, 250022, P. R. China. E-mail: ism_jiangxc@ujn.edu.cn

${ }^{b}$ School of Chemistry and Chemical Engineering, University of Jinan, No. 336 Nanxinzhuang West Road, Jinan, 250022, P. R. China.

E-mail:Chm_cuiy@ujn.edu.cn
}

an emerging technology that uses chromogenic materials to tailor the visible light transmission and thermal loads in buildings. Smart windows reduce energy consumption due to their astonishing energy harvesting ability and energy savings compared to the commonly used traditional glass. Therefore, according to the technological challenges, the aim of this green innovation is to build large-area, low-cost, durable, and multifunctional electrochromic (EC) smart windows.

It was first demonstrated in 1953 that $\mathrm{WO}_{3}$ thin films can change color via an applied voltage after being immersed in $\mathrm{H}_{2} \mathrm{SO}_{4}{ }^{1}$ Analogous results were achieved by Deb, at the
Shah Zeb received his Master's degree from the University of Peshawar, Pakistan, and enrolled as a PhD student in 2017, at the School of Chemistry and Chemical Engineering, Institute for Smart Materials \& Engineering, University of Jinan, Shandong, China. He has acquired skills in materials synthesis, advanced chemical techniques to control the morphology, surface chemistry and synthesis of functional nanoparticles, self-assembly of colloids into ordered structures, and low-cost and massive production of micro/nanoscale structures and different characterization techniques. His current research interest focuses on the synthesis and characterizations of tungsten oxide and its composites for gas sensing and electrochromic application in EC smart windows.
Guoxin Sun currently works as a Full Professor in the School of Chemistry and Chemical Engineering, Institute for Smart Materials \& Engineering, University of Jinan, China. He received his $P h D$ degree in 1998 from the Institute of Nuclear Research, Chinese Academy of Sciences. His current research activities focus on solvent extraction; synthesis of new extractants and their extraction removal of precious metals; and organic synthesis and functional nanomaterials. 
American Cyanamide Corporation in the $1960 \mathrm{~s}^{2}$ for $\mathrm{WO}_{3}$-based films, which were subsequently published in 1969 and 1973.,4 This work was motivated by large companies to produce informative displays and liquid crystal display devices. ${ }^{5}$ A similar concept was extended to rear-view mirrors in automobiles and industrialized. ${ }^{5}$ In the 1980 s, EC became widely accepted as energy-efficient technology. ${ }^{5-7}$ In 1984/1985, the name "smart windows" was coined, which become popular, not only to scientists but also soft media and the public. A recent development in chromogenic materials is their energysaving multi-step application in dynamic switchable smart glasses due to their unique electrochemical properties. A smart window is a dynamically switchable device in which the optical properties of chromogenic materials change upon stimulation, such as electricity, heat, and light, and can be observed. The corresponding technologies are known as photochromic, thermochromic, gasochromic and electrochromic windows, respectively. ${ }^{8-10}$ Among them, photochromic (PC) materials are the most attractive because they change color when exposed to light and are promising in many applications. The optical transmittance in PC materials drops and returns to its original state without stimulation, such as in sunglasses, lenses, and some plastics. ${ }^{11}$ In contrast to EC, PC films do not need an external power source. Recently, advancements in this area have been made using $\mathrm{WO}_{3}$ as an active material. ${ }^{12}$ However, utilizing $\mathrm{WO}_{3}$ in $\mathrm{PC}$ smart windows is limited because the switching largely depends on the light intensity and cannot be controlled by the user. ${ }^{11}$ However, this drawback can be overcome by hybrid photo-electrochromic materials that spontaneously work when exposed to sunlight

Yong Nie is an Associate Professor of Chemistry, Institute for Smart Materials \& Engineering, University of Jinan, China. In 2005, he received his $P h D$ in Inorganic Chemistry from the University of Heidelberg, Germany. After a short post-doctoral stay at the University of Colorado Boulder, he started his independent career in the School of Chemistry and Chemical Engineering, University of Jinan, China, in 2006. In 2014 he was a visiting scholar in the Department of Chemistry, University of Utah. In 2018, he transferred to the Institute for Smart Materials \& Engineering, University of Jinan. His current research focuses on boron clusters, luminescent materials and stimuli-responsive materials. and also change color or bleached with a supplied electric potential. ${ }^{11,13,14}$

Thermochromic (TC) materials can respond to changes in temperature with a clear and dark color. TC smart glasses display the automated capability of an optical transmission/ reflection with variations in temperature. Operationally, it cannot be a personal preference. Nonetheless, for smart functions, no additional energy is required. Many polymers and small molecules can be activated with thermally dependent reversible reactions or temperature-driven structural rearrangements. ${ }^{15}$ The most representative material in the group of TC materials is vanadium oxide $\left(\mathrm{VO}_{2}\right)$ or $\mathrm{VO}_{2}$-based composite materials, which allows the transmission of less heat energy at high temperatures than at low temperatures, and vice versa. ${ }^{16-23}$ An ultra-transparent coating composed of templatefree tunable $\mathrm{VO}_{2}$ hierarchical and honeycomb-like structures showed excellent visible light transparency up to $95.4 \%$ at $700 \mathrm{~nm}$ with a solar modulation ability of $5.5 \% .^{20}$ The obtained ultra-transparent honeycomb-like $\mathrm{V}_{2} \mathrm{O}_{5} / \mathrm{VO}_{2}$ film exhibited an enhanced anti-reflective and transmittance performance, which was demonstrated both theoretically and experimentally. The device constructed based on the ultra-transparent honeycomb-like $\mathrm{VO}_{2}$ coating film has potential for energysaving applications as smart windows. Similarly, vertically aligned $\mathrm{VO}_{2}$ hierarchical nanoplate structures grown on hollow spheroids without any template greatly enhanced the optical properties of $\mathrm{VO}_{2}$ film(s). ${ }^{22}$ The visible light transmission and solar heat modulation were recorded to be $50 \%$ and $11.2 \%$, respectively, with a contrast transmittance of $60{ }^{\circ} \mathrm{C}$ at $2500 \mathrm{~nm}$. The film thickness was effective in varying the visible transmittance by

Huiyan Xu works as a Lecturer in the Institute for Smart Materials \& Engineering, University of Jinan, China. In 2018, she obtained her PhD degree from the School of Materials Science and Engineering, Xi'an Jiaotong University, China. Meanwhile, she obtained another PhD degree from the Department of Physics, City University of Hong Kong, Hong Kong. Her research focuses on nanostructured functional materials and thin films. She is now studying energy saving EC smart coatings technology, including $\mathrm{VO}_{2}$ thin films.

Xuchuan Jiang currently works as a Full Professor at the Institute for Smart Materials \& Engineering at University of Jinan, China. Since the award of his PhD in 2001 from the University of Science and Technology of China (USTC), he has been devoted to studying the synthesis, self-assembly, and applications of functional nanoparticles. He has published over 140 papers in highly ranked international journals with a total of $>8000$ citations, giving him an h-index of 45. He has been worked in various academic research institutes since graduating from USTC, including the University of Washington (USA), University of Paris (France), University of New South Wales (Australia) and Monash University (Australia). 
lowering the solar optical modulation, i.e., $87.2 \%$, and solar optical sound of $5.3 \%$. The 2D hierarchical nanoplate structure possibly promotes the optical properties for smart windows.

Gasochromic (GC) windows aroused wide interest at the beginning of the 21st century due to their simple, inexpensive layer configuration, and high solar transmittance. The synthesis, characterization, and electrochemical activation of $\mathrm{WO}_{3}$ thin films used as cathodes in EC devices have also attracted interest in gasochromic applications. ${ }^{24} \mathrm{WO}_{3}$-based thin films are doped or decorated with a catalyst such as $\mathrm{Pd}$ or $\mathrm{Pt}$ nanoparticles. These films are transparent in the visible spectral range; however, upon exposure to $\mathrm{H}_{2}$ gas, the Pt and $\mathrm{Pd}$ nanoparticles in these systems promote the room temperature dissociation of the $\mathrm{H}_{2}$ molecules into hydrogen atoms, which upon reaction with the tungsten oxide support, provoke the coloration (i.e., blue color) of the oxide film. ${ }^{24}$ The dissociated $\mathrm{H}^{+}$ions and electrons spillover, and then these ion-electron pairs transfer to the $\mathrm{WO}_{3}$ layer and cause changes in the optical transmittance based on the small polaron transition mechanism. ${ }^{25}$ However, repeated coloring-bleaching cycles and long-term charge storing usually lead to structural dysfunctions and catalyst poisoning, which increase the switchable time and result in partial gasochromic irreversibility. ${ }^{26}$ The coloration in a photoemission experiment with a $\mathrm{Pt} / \mathrm{WO}_{3}$ system was observed, where $\mathrm{Pt}$ nanoparticle-decorated $\mathrm{WO}_{3}$ thin films were prepared by magnetron sputtering at an oblique angle and exposed to $\mathrm{H}_{2}$ gas in situ by near-ambient photoemission spectroscopy (NAPP). ${ }^{24}$ The formation of $\mathrm{W}^{n+}(n<6)$ species, which are associated with oxygen network vacancies, is believed to be responsible for the blue color when exposed to hydrogen. Given that magnesium ( $\mathrm{Mg}$ ) has a high hydrogen storage capacity, which tends to be further improved by hybridizing with various metals and oxides, it has been widely used in $\mathrm{H}_{2}$ storage and switchable mirrors. ${ }^{27}$ Due to the excellent catalytic property of $\mathrm{Nb}_{2} \mathrm{O}_{5}$ for the magnesium-hydrogen reaction, fluorocarbon (FC)/Pd/Mg- $\mathrm{Nb}_{2} \mathrm{O}_{5}$ switchable films fabricated via magnetron sputtering and low-temperature inductively coupled plasma chemical vapor deposition technology have demonstrated. ${ }^{28} \mathrm{FC} / \mathrm{Pd} / \mathrm{Mg}-\mathrm{Nb}_{2} \mathrm{O}_{5}$ films exhibit a high dynamic range of luminous properties, excellent optical modulation, and fast $\mathrm{H}_{2}$ adsorption/desorption performance. Similarly, $\mathrm{Mg}-\mathrm{TiO}_{2}$ thin films obtained via magnetron co-sputtering can act as the switchable layer in smart windows. Thus, gasochromic switchable mirrors based on $\mathrm{Pd} / \mathrm{Mg}-\mathrm{TiO}_{2}$ films fabricated via magnetron sputtering demonstrate excellent optical properties, microstructures, and strict structure-function relationships. ${ }^{29}$

EC materials ${ }^{30,31}$ have been extensively studied to adjust the transmittance, absorbance or reflectance of visible light and solar heat for energy-saving EC smart windows, information displays, self-dimming rare mirrors for automobiles and aircraft, electronic (e-)papers, electronic (e-)skins, etc. ${ }^{5,6,32-39}$ To satisfy different environments, the transparency and thermal insulation of windows are particularly tailored to reduce energy consumption, thereby decreasing cooling and heating costs. For this purpose, different types of EC materials have been designed and implemented in EC windows, including tin-doped indium oxide (ITO), aluminum-doped zinc oxide, and especially $\mathrm{WO}_{3}$ nanoparticles, which are electrochromic toward NIR light. ${ }^{40-43}$ Hybridizing $\mathrm{WO}_{3}$ with traditional transition metal oxides/organic electrochromics for visible light modulation makes it possible to construct dual/multifunction capacities that can independently modulate visible and NIR light. Polymer-based EC materials targeting visible light offer several potential advantages with good absorption properties in a wide range of colors, fast switching speed, and high coloration efficiency, which are usually difficult to achieve with metal oxides. ${ }^{43-45}$ Based on the design of the donor-acceptor (D-A) structure, ${ }^{46,47}$ EC polymers with a low onset potential and high reversible-switching stability including conjugated polymers, ${ }^{44,48-50}$ unconjugated polymers, ${ }^{51}$ and cross-conjugated polymers have been demonstrated..$^{52-54}$ Poly (3-hexylthiophene) (P3HT) is a regioregular semiconducting polymer that shows EC modulation under a small electrical bias polarity and acts as an anodically coloring material. ${ }^{55}$ Given that $\mathrm{WO}_{3}$ is a well-known cathodically coloring material, a hybrid composed of $\mathrm{P} 3 \mathrm{HT}$ and $\mathrm{WO}_{3}$ as two active electrochromics demonstrated a dual-band EC performance. ${ }^{55}$ In the dual EC device, P3HT was used as the anode coloring material and $\mathrm{WO}_{3}$ as the cathode coloring material, and therefore, the transfer of charges from $\mathrm{WO}_{3}$ to $\mathrm{P} 3 \mathrm{HT}$ makes it colored, whereas the colors of both materials were bleached in the opposite direction. ${ }^{55}$

To achieve multiple colors and enhance the functionality of all-in-one-type EC devices, the incorporation of several EC chromophores is inevitable. ${ }^{56}$ They can achieve the simplest device configuration because of their facile fabrication using versatile solution processes (e.g., spin coating and printing) and easily tunable device characteristics such as transmittance contrast, color, operating voltage, and cyclic stability by varying the concentration of the chromophores ${ }^{57,58}$ and tuning the electrochemical properties ${ }^{59}$ or molecular structures ${ }^{60,61}$ of the redox species. Accordingly, 1,1'-disubstituted-4,4'bipyridinium salts, which are known as viologens, have been extensively studied because their optical and electrochemical properties can be easily tuned by modifying their substituents. The coloration potential (i.e., reduction potential) of viologens depends on the color. Therefore, uneven color contrast is obtained when a fixed voltage is applied to a device including multiple viologens. ${ }^{62,63}$ However, self-bleaching occurs even under open-circuit conditions, which is considered to be a weakness of all-in-one EC devices. Polymeric viologens (polyviologens) have been demonstrated to lower the diffusivity of EC chromophores and minimize self-bleaching. ${ }^{64}$ Compared with EC devices based on mono-viologens corresponding to the monomer of poly-viologens, EC devices containing polyviologens exhibit the advantages of lower coloration voltage $(-0.55 \mathrm{~V})$ and higher coloration/bleaching cyclic stability ( $>1500$ cycles). In particular, as-designed poly-viologen ECDs show remarkably reduced self-bleaching, resulting in extremely low power consumption $\left(\sim 8.3 \mu \mathrm{W} \mathrm{cm}^{-2}\right)$ to maintain the colored state. ${ }^{64}$ Moreover, compared with common glass, poly-viologen-based EC devices have strong NIR ray absorption capacity, which proves their feasibility as effective heat shutters at low-power consumption. ${ }^{64}$ EC smart windows utilizing these 
composite materials can simultaneously control building lighting and heating.

In the OFF state, EC devices are transparent to natural light. When a suitable voltage is applied, electrons are transferred due to the oxidation-reduction reaction, and the EC materials become colored with an optical modulation. Thus, by varying the voltage applied across electrodes modified with EC materials or their composites, they can be switched between "bright and warm", "bright and cool", and "dark and cool" modes. Consequently, at a lower potential, windows are in the cooling mode, blocking heat flux but allowing UV light, whereas setting smaller potential switches to dark mode limits both heat rays and UV light. These functional modes facilitate the multipurpose use of windows as follows: (i) operate under different weather conditions particularly with personal preference, (ii) provide better comfort for occupants, and (iii) features associated with energy-storage/harvesting, aseptic/sterile function, aesthetics, privacy/security, high-latitude zones, aircraft, etc. $5,32,33,65,66$

Energy efficiency in buildings has become a focus, given that $40 \%$ of the global energy consumption comes from the urban environment. ${ }^{67,68}$ Due to the rapid increase in global warming, building cooling is becoming a key problem because every $1{ }^{\circ} \mathrm{C}$ rise in temperature leads to a $20 \%$ increase in energy consumption. ${ }^{67}$ Building cooling systems in developed countries account for nearly $50-60 \%$ of the total building energy consumption. ${ }^{8,67}$ The concept of a "zero-energy building" has emerged to control the thermal load via the dynamic optical modulation of "smart" windows. ${ }^{69}$ Moreover, these Wi-Ficonnected tint-controllable smart glass surfaces are equipped with environmental sensors that can read room occupancy, weather, and sunlight. The emerging smart windows installed in commercial and domestic areas can save $\sim 170 \mathrm{~kW} \mathrm{~h}$, i.e., $30 \%$ of the total energy consumption annually, which is an important step towards achieving "zero energy buildings". 8,67 The other recent approaches based on energy-saving EC materials and technologies for novel multifunction are published elsewhere. ${ }^{67,68,70-78}$ Modern commercial buildings in European countries have installed functioning EC smart windows instead of active air conditioning, and approximately $4.5 \%$ energy can be saved annually in the USA and Australia. ${ }^{79}$ Similarly, autodimming smart glass and automotive rear-view mirrors have been commercialized. ${ }^{80}$

Nonstoichiometric metal oxides such as $\mathrm{WO}_{3-x}, \mathrm{MoO}_{3-x}$, $\mathrm{TiO}_{x}, \mathrm{NbO}_{x}, \mathrm{NiO}_{x}, \mathrm{~V}_{x} \mathrm{O}_{y}, \mathrm{MnO}_{x}$, and $\mathrm{Cu}_{x} \mathrm{~S}_{y}$ have strong light absorption/reflection and thermal insulation properties. In particular, $\mathrm{WO}_{3-x}$-based materials in energy-saving EC devices have application prospects because (i) they possess unusual defects and enormous oxygen vacancies, thus achieving strong adsorption affinity with electrolyte ions, (ii) oxygen vacancies provide free electrons, which increase the electric conductivity, resulting in quick electron transfer from the surface, (iii) strong local surface plasmonic resonance (LSPR) provides strong photoabsorption over a wide range of the NIR region, and (iv) a significant reduction in bandgap results in strong light absorptivity, generating electron-hole pairs. ${ }^{81-86}$ Because of their unique channel/tunnel-like structures for easy electrolyte ion intercalation, defective structure, low cost, high natural abundance, excellent thermal stability, good compatibility, and high theoretical capacitance, oxygen-deficient $\mathrm{W}_{18} \mathrm{O}_{49}$ nanostructures are attractive for application in various chromogenic devices, particularly in EC smart windows. ${ }^{82,83,87,88}$

The scope of this review is focused on the recent advancements in the synthesis of $\mathrm{WO}_{3-x} / \mathrm{WO}_{3}$ nanostructures, EC smart coatings, and future advances in smart windows. Sections 1, 2 and 3 briefly introduce the basic properties and EC mechanism of sub-stoichiometric $\mathrm{WO}_{3-x}$. Section 4 provides an overview of the synthetic methods, film formation, and EC properties. Section 5 discusses the strategies for improving the electrochromism of tungsten oxide, including hybridization, elemental/plasmonic doping, and surface decoration. Section 6 describes the all-in-one solid-state next-generation multifunctional EC window principle and its configuration as a typical example for realization, followed by the conclusions, existing and prospective challenges, and finally future demands.

\section{Electrochromism}

EC materials coated on a transparent conducting electrode can change their optical properties reversibly and continuously by applying a small electric potential. Thus, the transport of ions can occur between the EC material film(s), ion storage/ conducting film(s), and/or ion conducting electrolyte(s). The charges are balanced by intercalated/deintercalated electrons, and then transported through the transparent conductors. Smart films can maintain their physical properties with reversible optical modulation in the open circuit, and their color can be refreshed to a specific intermediate state. If energy storage devices can sense energy changes in predictable patterns, we can quickly determine that energy is running out before these devices stop working, demonstrating a wide range of potentially smart applications. Moreover, energy can be saved by using the energy stored in EC devices. If we need to color an EC device to resist sunshine/protect privacy, charges can be stored inside. If the coloration is no longer needed, the stored energy can be released through an external circuit, providing another way for energy resource utilization.

As mentioned, EC films and the complementary counter electrode may have similar optical properties. In a hybrid dualEC device, two complementary electrodes (working and counter) coated with switchable EC materials have sufficient charge capacity for ion storage, as shown in Fig. 1. These EC devices are promising because the redox reaction that occurs in the active layer is fully compensated and can complete the color changes, resulting in an electrochemically stable batterytype cell. Therefore, EC materials can be (i) cathodic EC materials and (ii) anodic EC materials. Some EC materials that can achieve color from transparent to dark colors by ioninsertion/reduction during cathodic polarization are called cathodic EC materials. Similarly, some are anodic electrochromics, which can achieve a dark color/opaque state from a 


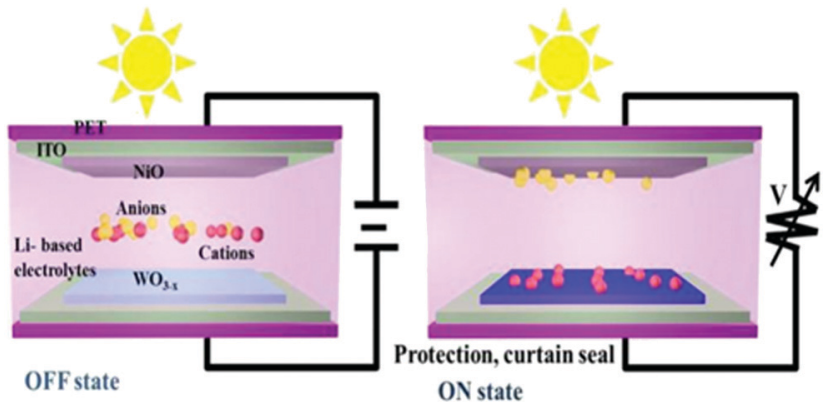

Fig. 1 Simplified EC smart window illustration in the colored and bleached state. Electron intercalation through an external circuit into the EC materials (cathode and/or anode), while ions flow through the electrolyte to compensate for the electronic charges. The panel shows sealing with a curtain for protection.

transparent state under ion-extraction/oxidation during the anodic polarization process. The key variables for evaluating the performance of EC materials are as follows: (i) optical modulation, EC materials should carry excellent optical modulation $(100 \%)$, i.e., fully transparent in the bleached state and fully opaque in the dark state. (ii) Switching speed, EC materials should reach $90 \%$ optical modulation between the coloration and bleaching states. (iii) Coloration efficiency is the key index to evaluate the EC performance. A high coloration efficiency ensures that materials exhibit large optical modulation with low power consumption. (iv) The cycling stability and reliability of EC materials should be maintained for more than 50 years without degradation during use.

Many inorganic materials such as $\mathrm{TiO}_{2}, \mathrm{WO}_{3}, \mathrm{MoO}_{2}, \mathrm{VO}_{2}$, $\mathrm{Nb}_{2} \mathrm{O}_{5}, \mathrm{Ta}_{2} \mathrm{O}_{5}, \mathrm{CeO}_{2}, \mathrm{NiO}, \mathrm{Co}_{3} \mathrm{O}_{4}$, and $\mathrm{SnO}_{2}{ }^{89-92}$ organics such as Prussian blue; and small organic molecules ${ }^{93-97}$ including conjugated polymers, ${ }^{96-99}$ transition metal complexes, ${ }^{34,40,79,100-104}$ viologens ${ }^{105}$ and conducting polymers ${ }^{106-109}$ have been examined recently. The two dominant EC materials are polymers and metal oxides. ${ }^{10}$ Polymers are excellent candidates for bendable, foldable, and stretchable applications because of their flexible molecular framework interjunctions. ${ }^{10,110,111}$ Metal oxides possess ultrasmall particle sizes with a large specific surface area and are expected to exhibit excellent EC functionality, possibly due to their short ion diffusion length, easy electrolyte accessibility, excellent coloration efficiency, cyclic stability, and durability. However, metal oxide-based films also have certain disadvantages, such as cracking and degradation, after continuous bending and stretching.

Organic EC materials exhibit controllable color, higher coloration efficiency, and polyelectrochromism similar to that of inorganic EC materials. Particularly, the well-known organic EC materials are organic small molecules, especially viologens, which are the major candidates used in EC display devices. Specifically, viologens are 1,1'-disubstituted-4,4'-dipyridinium salts, with two positive charges, which are colorless in the normal state $\left(\mathrm{V}^{2+}\right)$, deep-blue in the radical cation state $\left(\mathrm{V}^{+}\right)$and reversible. ${ }^{39}$ When the $\mathrm{N}$-substituents are short alkyl chains (e.g., methyl or ethyl), the colored state including the viologen cation radical shows a blue color. As the alkyl length increases (e.g., heptyl) and the electrolyte has hydrophilic character, the colored species tend to dimerize. Consequently, the colored state is magenta, but the quasi-reversible oxidation of the dimerized viologen cation radical degrades the device performance. Various aryl-substituents have been also reported to obtain other colors such as green. Based on the numerous viologens, multicolor ECDs with EC ion gels have been demonstrated. ${ }^{112}$ The EC properties of conjugated conducting polymers such as polythiophenes (PT), polydimethylsiloxane (PDMS), poly(vinyl alcohol) (PVA), poly(methyl methacrylate) (PMMA), polyaniline (PANI), polyimide (PI), polypyrroles (PPy), polycarbazoles $(\mathrm{PCz})$, and polyfurans $(\mathrm{PF})$ vary with their molecular structure and bandgap energy. The coloration may be due to the donor moiety, which accelerates the $\pi-\pi^{*}$ electronic transition. The HOMO energy level is at a higher energy state. In contrast, the acceptor moiety lowers the LUMO energy level, promoting the charge transfer between the two types of units within the backbone of the polymer in the electrolyte upon electrochemical oxidation and reduction. ${ }^{113}$ PT, PANI, and PPy have gained intense research interest due to their facile synthetic route, low redox potential and suitable bandgap. The electrochromism in conjugated polymers is tunable with changes in their bandgap, monomer type, conjugation length, side-chain/substitution groups, steric effects, and stereoregularity. Because of their unique absorptivity and redox reactions in the visible range, they change their electronic structure, optical states, and conductivity under an applied electric current. Metal-organic frameworks (MOFs) are porous materials, and their permanent porosity offers channels for the fast transport of ions, thus enhancing their EC properties. ${ }^{13}$ However, there are certain challenges ${ }^{9,39,110}$ that limit the largescale preparation of organic EC materials as follows:

(i) their multistep synthesis and purification processes,

(ii) use of hazardous organic reagents,

(iii) their photo-electrochemical instability,

(iv) their optical contrast fade-out caused by overoxidation, and

(v) wide range optical modulation in the visible region and lack of control over NIR light.

Recent interest in flexible, stretchable, and foldable devices has been focused on incorporating EC metal oxides in polymers to prepare hybridized materials by carefully matching their refractive index and surface chemistry. The resulting hybrid composites exhibit improved chemical and mechanical properties, enhanced dispersion of nanoparticles, stimuliresponsiveness and provide excellent bendability, stretchability, and foldability. ${ }^{10,39,83,86,98,114}$ These intrinsic stretchable and transparent conductive electrochromics combine to balance the optical transparency, conductivity, and mechanical stretchability. Focusing on the explosive growth of metal oxides towards EC applications, tungsten, titanium, niobium, vanadium, molybdenum, nickel, copper, manganese, cobalt, and iridium oxides are highly addressed based on their preparation, stoichiometry, particle morphology, composition, and deposition process. Recently, $\mathrm{WO}_{3} / \mathrm{WO}_{3-x}$ has been demonstrated to be an ideal material owing to its unique EC, electrochemical, and optical properties. The unique detail of this review is the 
Table 1 Advanced EC materials and their mechanisms and merits towards EC smart glass, evaluated for the desired "zero-energy buildings" "smart windows"

\begin{tabular}{|c|c|c|c|c|c|}
\hline EC material & EC mechanism and properties & $\begin{array}{l}\text { Coloration effi- } \\
\text { ciency }\left(\mathrm{cm}^{2} \mathrm{C}^{-1}\right)\end{array}$ & $\begin{array}{l}\text { Transmittance } \\
\text { contrast }\end{array}$ & $\begin{array}{l}\text { Switching speed (color/ } \\
\text { bleach (s)) and cycle life } \\
\text { (cycles) }\end{array}$ & Ref. \\
\hline $\mathrm{WO}_{3-x}$ (amorphous) & $\begin{array}{l}\text { Ion insertion, polaron absorption, charge } \\
\text { transfer, and Vis and NIR absorption }\end{array}$ & $125-80$ & $\begin{array}{l}56-70 \% \text { at } \\
550-800 \mathrm{~nm}\end{array}$ & $5 \mathrm{~s} / 2.5 \mathrm{~s}, 1000$ to 4000 & $\begin{array}{l}115- \\
118\end{array}$ \\
\hline $\begin{array}{l}\mathrm{WO}_{2.72} \text { assembled on } \mathrm{Ag} \\
\text { nanowires }\end{array}$ & $\begin{array}{l}\text { Ion insertion, polaron absorption, charge } \\
\text { transfer, and Vis and NIR absorption }\end{array}$ & 35.7 & $58-86 \%$ at $550 \mathrm{~nm}$ & $2 \mathrm{~s} / 4 \mathrm{~s}, 1000$ & 119 \\
\hline Mesoporous $\mathrm{WO}_{3}$ & $\begin{array}{l}\text { Ion intercalation, polaron absorption, charge } \\
\text { storage, Vis NIR absorption }\end{array}$ & 188 & $76 \%$ at $700 \mathrm{~nm}$ & $0.8 \mathrm{~s} / 0.4 \mathrm{~s} 1000$ & 120 \\
\hline $\mathrm{WO}_{3}$ (crystalline) & $\begin{array}{l}\text { Ion intercalation, polaron absorption, charge } \\
\text { transfer, free carrier, and Vis and NIR } \\
\text { absorption }\end{array}$ & 42 & $50-70 \%$ at $550 \mathrm{~nm}$ & Several min, 3000 & 101 \\
\hline $\mathrm{WO}_{3} \cdot 2 \mathrm{H}_{2} \mathrm{O}$ nanoplates & $\begin{array}{l}\text { Pseudocapacitive, polaron absorption, charge } \\
\text { transfer, and NIR absorption with transmit- } \\
\text { tance modulation }(90.4 \%)\end{array}$ & 322.6 & $\begin{array}{l}63.8 \% \text { at } 550 \mathrm{~nm} \\
\text { and } 90.4 \% \text { at } \\
1600 \mathrm{~nm}\end{array}$ & $93.7 \%$ after 500 cycles & 121 \\
\hline $\mathrm{WO}_{3-x} / \mathrm{POMs}$ & $\begin{array}{l}\text { Ion insertion, polaron absorption, charge } \\
\text { transfer, and Vis, NIR selective absorption }\end{array}$ & 42.31 & $\begin{array}{l}64 \% \text { at } 630 \mathrm{~nm} \text { and } \\
88 \% \text { at } 915 \mathrm{~nm}\end{array}$ & $26 / 86,500$ & 122 \\
\hline $\begin{array}{l}\mathrm{WO}_{2.72}-\mathrm{NbO}_{x} \text { on ITO- } \\
\text { PET }\end{array}$ & $\begin{array}{l}\text { Ion insertion, polaron absorption, charge } \\
\text { transfer, capacitive, and Vis and NIR selective } \\
\text { transmission }\end{array}$ & 30 & - & Mint, 2000 & 123 \\
\hline $\begin{array}{l}\mathrm{MoO}_{3}-\mathrm{WO}_{3} / \mathrm{Ag} / \mathrm{MoO}_{3}- \\
\mathrm{WO}_{3}\end{array}$ & $\begin{array}{l}\text { Ion diffusion, charge transfer, and Vis and NIR } \\
\text { selective transmission }\end{array}$ & 70 & $\begin{array}{l}72.9-79.3 \text { at } 400- \\
800 \mathrm{~nm}\end{array}$ & $2.7 / 4.1,500$ & 124 \\
\hline $\mathrm{WO}_{3} / \mathrm{Ag} / \mathrm{WO}_{3}$ & $\begin{array}{l}\text { Ion diffusion, charge transfer, and Vis and NIR } \\
\text { selective transmission }\end{array}$ & 30.5 & $\begin{array}{l}52.5-82.7 \text { at } 400- \\
780 \mathrm{~nm}\end{array}$ & $15 / 22.4,2500$ & 125 \\
\hline $\mathrm{NiO}$ & $\begin{array}{l}\text { Pseudocapacitive (anodic) polaron absorption, } \\
\text { and charge transfer }\end{array}$ & $30-100$ & $68 \%$ at $650 \mathrm{~nm}$ & 53,10000 & 117 \\
\hline $\begin{array}{l}\text { Smart window for zero } \\
\text { energy building }\end{array}$ & $\begin{array}{l}\text { Capacitive, sensing, plasmon absorption + } \\
\text { polaron/charge transfer, and Vis and NIR- } \\
\text { independent absorption + reduce toxicity }\end{array}$ & Infinite & $\begin{array}{l}\text { More than } 75 \% \text { at } \\
550-630 \mathrm{~nm} \text { and } \\
\text { More than } 95 \% \text { at IR } \\
\text { region }\end{array}$ & $\begin{array}{l}\text { Seconds/less } \\
\text { Greater than } 50000 \\
\text { Stand for over } 50 \text { years }\end{array}$ & $?$ \\
\hline
\end{tabular}

prospective advancement towards next-generation smart windows using $\mathrm{W}_{18} \mathrm{O}_{49} / \mathrm{WO}_{2.72}$ among the metal oxide (Table 1).

\section{Nonstoichiometric $\mathrm{WO}_{3-x}$ : properties and electrochromic mechanism}

\subsection{Properties}

Tungsten oxide $\left(\mathrm{WO}_{3} / \mathrm{WO}_{3-x} / \mathrm{WO}_{3} \cdot x \mathrm{H}_{2} \mathrm{O}\right)$ is a type of perovskite structure formed of $\mathrm{WO}_{6}$ octahedra connected by $\mathrm{W}-\mathrm{O}-\mathrm{W}$ bonds or water molecule(s) similar to the geometry of rhenium oxide $\left(\mathrm{ReO}_{2}\right)$. Its electronic structure is composed of oxygen with a filled $2 \mathrm{p}$ orbital (valence band) and tungsten with an empty $5 \mathrm{~d}$ orbital (conduction band). ${ }^{126,127}$ The $\mathrm{WO}_{6}$ octahedron can be interpreted as a central tungsten metal surrounded by six equidistant oxygen atoms, sharing edges or corners, thus forming a 3D-octahedral crystal structure. ${ }^{128}$ In the octahedral geometry, there are hexagonal tunnels and trigonal windows (Fig. 3), and the corner sites facilitate the transport of ions. One or two charge-compensated electrons are transferred to the adjacent $\mathrm{W}$-atoms, reducing them to $\mathrm{W}^{5+}$ ions. ${ }^{129}$ The chargecompensated electrons can be transferred to two neighboring $\mathrm{W}$ ions to form a doubly charged $\mathrm{W}^{4+}$ and transition between $\mathrm{W}^{4+}$ to $\mathrm{W}^{5+} \cdot{ }^{129-131}$ The crystal lattice of $\mathrm{WO}_{x}$ contains considerable oxygen deficiency sites with partially reduced $\mathrm{W}^{5+}$ species. These sub-oxides with substantial oxygen vacancies become more metalized with an increase in non-stoichiometry and achieve high conductivity. The $\mathrm{WO}_{6}$ octahedral symmetry undergoes tilting and rotation with a change in temperature, ${ }^{132}$ forming typical monoclinic, hexagonal, orthorhombic and tetragonal crystal symmetries, indicating that the $\mathrm{WO}_{3}$ crystal phases are partially reversible. At room temperature $\left(25^{\circ} \mathrm{C}\right)$, $\mathrm{WO}_{3}$ crystallizes in the triclinic phase, which transforms into other phases upon annealing, ${ }^{132}$ such as monoclinic II $\left(\varepsilon-\mathrm{WO}_{3}\right.$, $\left.<-43{ }^{\circ} \mathrm{C}\right) \rightarrow \operatorname{triclinic}\left(\delta-\mathrm{WO}_{3},-43{ }^{\circ} \mathrm{C}\right.$ to $\left.17{ }^{\circ} \mathrm{C}\right) \rightarrow$ monoclinic I $\left(\gamma-\mathrm{WO}_{3}, 17{ }^{\circ} \mathrm{C}\right.$ to $\left.400{ }^{\circ} \mathrm{C}\right) \rightarrow$ orthorhombic $\left(\beta-\mathrm{WO}_{3}, 400{ }^{\circ} \mathrm{C}\right.$ to $\left.740{ }^{\circ} \mathrm{C}\right) \rightarrow$ tetragonal $\left(\alpha-\mathrm{WO}_{3},>740{ }^{\circ} \mathrm{C}\right)$, as shown in Fig. 2 . The obtained crystal structures are orthorhombic for $\mathrm{W}_{3} \mathrm{O}_{8}$ and $\mathrm{W}_{32} \mathrm{O}_{84}$, monoclinic for $\mathrm{W}_{17} \mathrm{O}_{47}, \mathrm{~W}_{18} \mathrm{O}_{49}, \mathrm{~W}_{20} \mathrm{O}_{58}$, and $\mathrm{W}_{25} \mathrm{O}_{73}$, and tetragonal for $\mathrm{W}_{5} \mathrm{O}_{14}$. The reduction reactions of monoclinic $\mathrm{WO}_{3}\left(\gamma-\mathrm{WO}_{3}\right)$ forms Magneli phases. Monoclinic $\mathrm{WO}_{3}$ is the most stable phase studied as EC films in EC devices. In this reduction process, with an increase in oxygen vacancies, the position of the $\mathrm{WO}_{6}$ octahedra in the crystal structure changes from corner-sharing to edge-sharing, which split by wellordered crystallographic shear planes. ${ }^{132}$ In $\mathrm{WO}_{3-x}$, the edgesharing $\mathrm{WO}_{6}$ octahedra with channels arrange to form hexagonal and pentagonal tunnels.

The nonstoichiometric $\mathrm{WO}_{3-x}$ was first demonstrated by Glemser and Sauer, where they found that the pure stoichiometric $\mathrm{WO}_{3}$ phase structure could be converted to $\mathrm{WO}_{2.9}$ by introducing oxygen vacancies. ${ }^{133}$ Therefore, the $\mathrm{WO}_{3-x}$ lattice can exhibit a large number of oxygen vacancies, resulting in a variety of non-stoichiometric compounds. ${ }^{79,134}$ Recent research has focused on the synthesis of sub-stoichiometric tungsten oxide $\left(\mathrm{WO}_{3-x}, 0<x<1\right)$, including $\mathrm{W}_{20} \mathrm{O}_{58}(x=2.90)$, $\mathrm{W}_{19} \mathrm{O}_{55}(x=2.89), \mathrm{W}_{5} \mathrm{O}_{14}(x=2.80), \mathrm{W}_{18} \mathrm{O}_{49}(x=2.72)$, and 


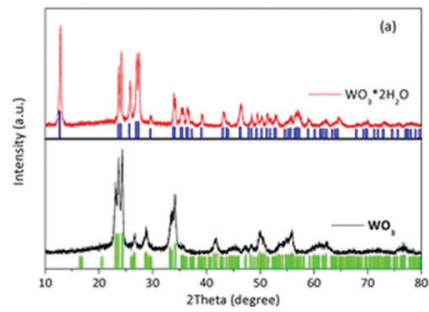

(d)

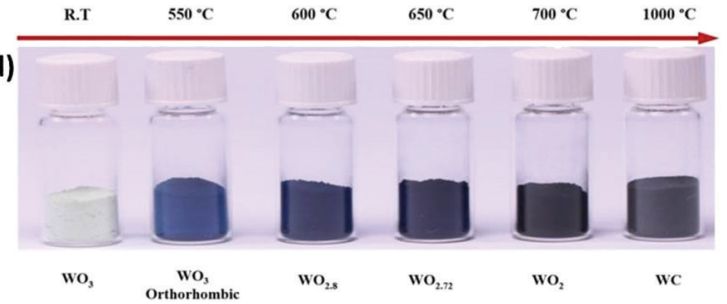

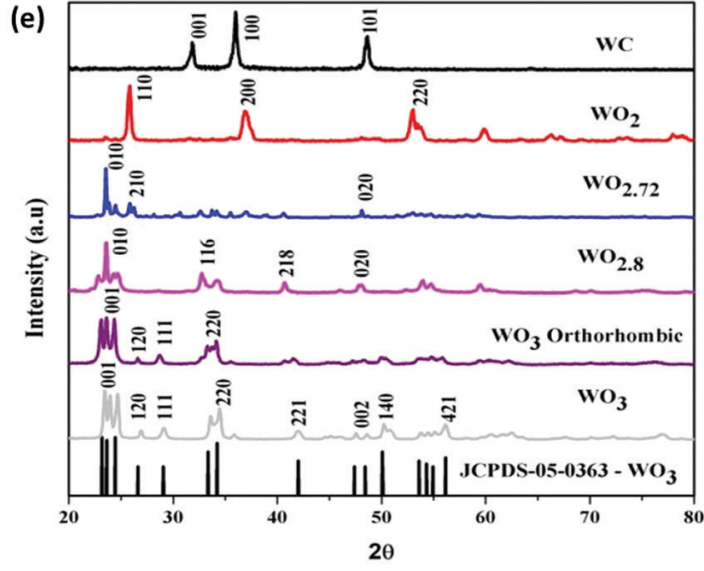

Fig. 2 (a) XRD patterns and crystal structures of (b) $\mathrm{WO}_{3}$ and (c) $\mathrm{WO}_{3} \cdot 2 \mathrm{H}_{2} \mathrm{O}$ (W, blue; $\mathrm{O}$, red; and $\mathrm{H}$, white). ${ }^{157}$ (d) Variations in the color of tungsten oxide powders prepared via reduction under a $\mathrm{CO}$ atmosphere at different temperatures. (e) XRD patterns of pure tungsten trioxide before reduction and its sub-oxides after reduction under a CO atmosphere. Reproduced with permission. ${ }^{158}$ Copyright@2017, MDPI.
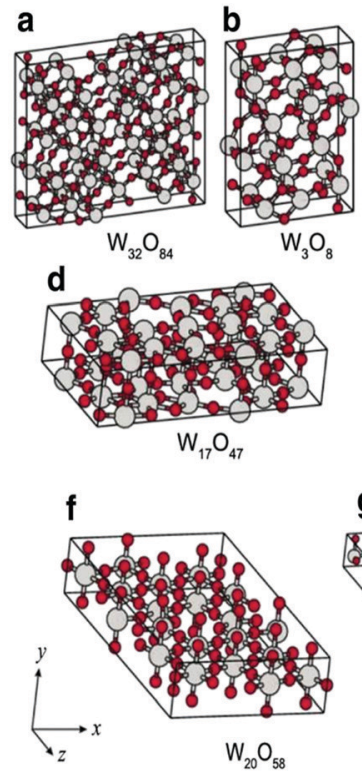

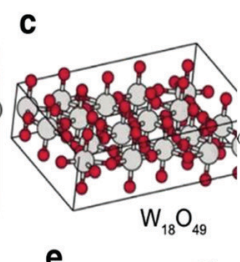

e
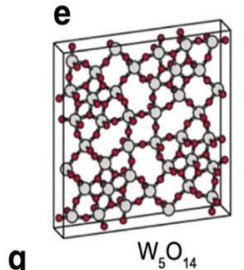

g

$\mathrm{W}_{5} \mathrm{O}_{14}$

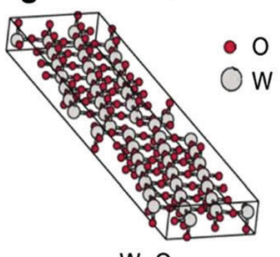

$\mathrm{W}_{25} \mathrm{O}_{73}$
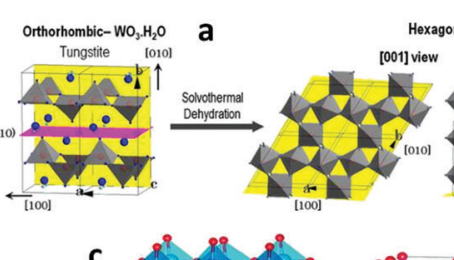

[001] view [100] vie

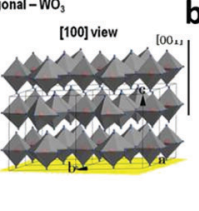

b ond

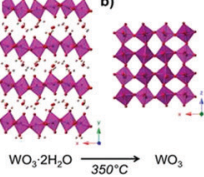

c
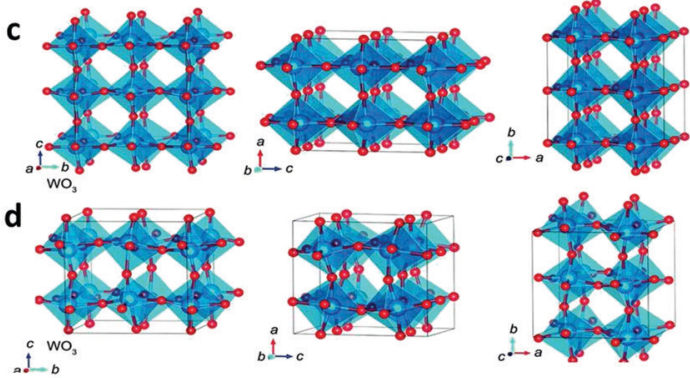

e
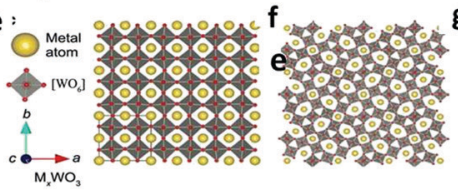

g sporonozes $\odot$ cs

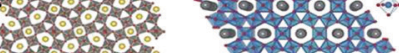

3000 \%

b 9.0\%0.

Fig. 3 Left: $(\mathrm{a}-\mathrm{g})$ Unit cells of various $\mathrm{WO}_{3-x}$. The large gray and small red balls represent $\mathrm{W}$ and $\mathrm{O}$ atoms, respectively. Reprinted with permission. ${ }^{165}$

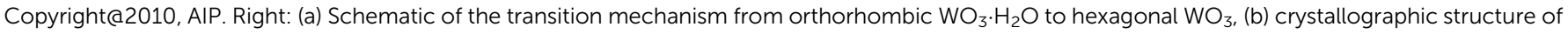
monoclinic $\mathrm{WO}_{3}$ from layered hydrated $\mathrm{WO}_{3}$ via heat treatment process: hydrated $\mathrm{WO}_{3}\left(\mathrm{WO}_{3} \cdot 2 \mathrm{H}_{2} \mathrm{O}\right)$ to monoclinic $(\gamma)$ WO 3 . Reproduced with permission. ${ }^{161}$ Copyright $\left(\mathrm{C} 2017\right.$, ACS. (c) and (d) Unit cell orientations of orthorhombic and monoclinic $\mathrm{WO}_{3}$ along the $\mathrm{a}, \mathrm{b}$, and $\mathrm{c}$ planes, ${ }^{166}$ and tungsten bronze $\left(\mathrm{M}_{x} \mathrm{WO}_{3}\right)$ (e) cubic $(x=1)$, (f) tetragonal $(1>x>0.33),{ }^{167,168}$ and $(\mathrm{g})$ hexagonal $(x<0.33)$ structures. $^{168}$

$\mathrm{W}_{32} \mathrm{O}_{84}(x=2.62) .{ }^{135} \mathrm{~W}_{18} \mathrm{O}_{49}$ is an $n$-type material semiconductor with a bandgap of 2.6-3.5 eV. The formation of oxygen vacancies in the $\mathrm{WO}_{x}$ lattice affects the Fermi level of tungsten oxide, narrows the bandgap, and increases the conductivity and free carrier electrons. ${ }^{79,133,136}$ These materials are usually insoluble in all solvents and can be oxidized to stoichiometric $\mathrm{WO}_{3}$ after a long oxidation reaction. Decreasing the particle size increases the bandgap and results in a blue shift and photon absorption. ${ }^{88,136}$
$\mathrm{WO}_{3-x}$ is an interesting LSPR candidate host due to its distinctive properties with outer d-valence electrons. The new discrete energy bands beneath the conduction band (Fig. 4b) are produced by oxygen vacancies. ${ }^{81,137,138}$ Due to the presence of oxygen vacancies, crystal defects, or insertion of tiny metal ions, strong LSPR with energy comparable to the bandgap energy can induce significant near-infrared absorption properties. The LSPR peak in plasmonic nanoparticles is directly proportional to the square root of the density of free electrons 


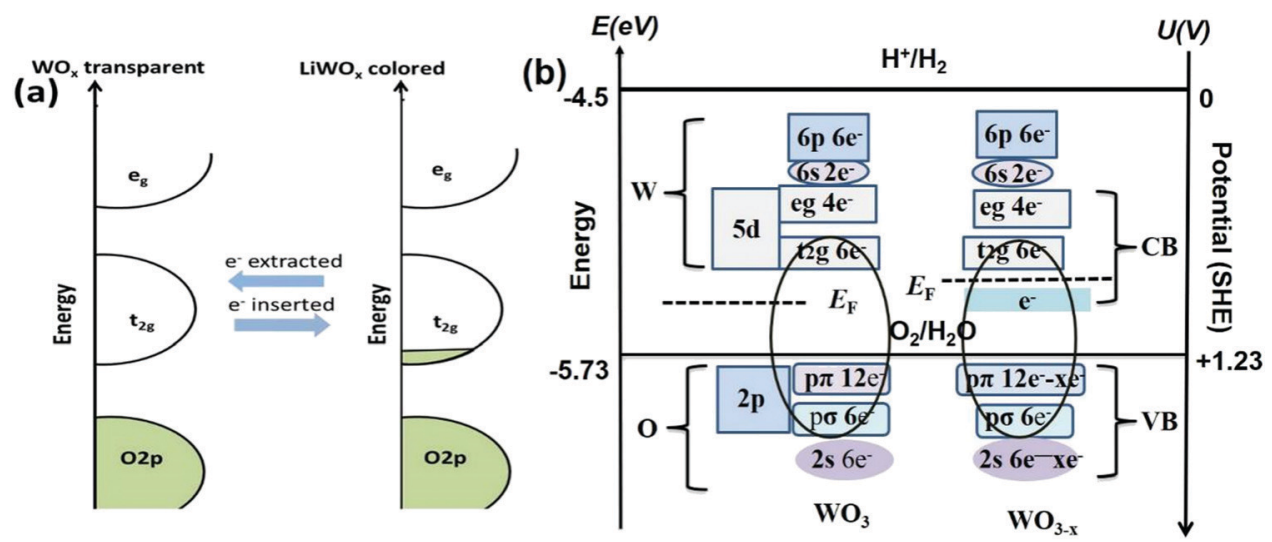

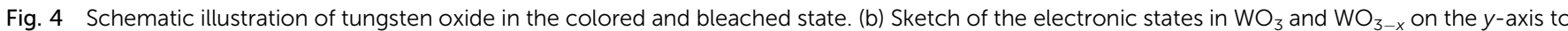
the left in energy $(\mathrm{eV})$ and the right in potential according to the standard hydrogen potential (SHE) under vacuum. Reproduced with permission. ${ }^{189}$ Copyright@2017, Elsevier

in the particles. ${ }^{139}$ The LSPR depends on certain factors, such as the particle size, dimensions, structure, shape, and dielectric properties of metals. ${ }^{140}$ Controlled heat treatment in a weak reducing environment is a facile but effective way to increase the number of oxygen vacancies. The dopants/desired impurities donate their electrons/holes and increase the density of free electron(s) in the conduction band. Meanwhile, the LSPR generates a rigorous local electric field, promoting the photoelectric harvesting application, which has broad application prospects for smart functions in EC windows. ${ }^{141}$

The monoclinic $\mathrm{W}_{18} \mathrm{O}_{49}$ has excellent stability and can be recovered in the pure phase with substantial oxygen vacancies. $\mathrm{W}_{18} \mathrm{O}_{49}$ possesses both the octahedral and pentagonal bipyramidal coordination links of the $\mathrm{W}$ with $\mathrm{O}$ atoms. Similar to $\mathrm{h}$ $\mathrm{WO}_{3}$, ordered edge-sharing $\mathrm{WO}_{6}$ networks produce three different tunnels/cavities on a crystal plane, such as hexagonal, trigonal, and quadrilateral, to accommodate guest ions during the electrochemical process (Fig. 3). ${ }^{142}$ Due to its astonishing defect structure and remarkable physicochemical properties, $\mathrm{W}_{18} \mathrm{O}_{49}$ has become a potential candidate for application in solar cells, ${ }^{143}$ photocatalysis, ${ }^{144-147}$ chromogenic devices, ${ }^{119,139}$ photothermal agents for cancer treatment, ${ }^{148}$ supercapacitors and batteries for energy storage ${ }^{133}$ and sensors toward various toxic gas molecules because of its distinctive structural faces, ultrathin nature, small particle size, high surface area, reproducible morphologies, crystal defects, and oxygen vacancies. ${ }^{149-154}$ Hexagonal $\mathrm{WO}_{3}\left(\mathrm{~h}-\mathrm{WO}_{3}\right)$ is a metastable phase that has received significant interest in electrochemical applications as an $\mathrm{Li}^{+}$and $\mathrm{Na}^{+}$ion host material, ${ }^{155}$ and its crystal structure is presented in Fig. 3a. In other polymorphs, the octahedral units are organized into a perovskite-like structure. In hexagonal $\mathrm{WO}_{3}$, the $\mathrm{WO}_{6}$ octahedra existing in the form of three- and six-membered rings in the " $a b$ " plane create trigonal cavities and hexagonal windows, respectively, whereas in the " $c$ axis", sharing of the axial oxygen results in 4-coordinated square windows. ${ }^{156}$ These tunnels offer accommodation sites for a large number of guest cations during the electrochemical process. No hexagonal phase was observed during the structural transformation; however, it can be obtained via the dehydration of hydrated tungsten oxides, indicating that the structural transformation can only occur in aqueous media. In addition, it can be converted into a monoclinic phase (Fig. 3b) by annealing at a temperature above $400{ }^{\circ} \mathrm{C}$. The redox reactions of tungsten metal/tungsten oxides in aqueous electrolyte are controlled by various $\mathrm{pH}$-related half-reactions, and possible explanations can be found in the review published by Shinde and Jun. ${ }^{133}$

Tungsten oxide hydrates $\left(\mathrm{WO}_{3} \cdot \mathrm{xH}_{2} \mathrm{O}\right)$ also exhibit properties similar to $\mathrm{WO}_{3-x}$ nanostructures. They can be classified into subclasses based on the number of water molecules present in their crystal lattices as follows:

(i) $\mathrm{WO}_{3}$-dihydrate $\left(\mathrm{WO}_{3} \cdot 2 \mathrm{H}_{2} \mathrm{O}\right)$,

(ii) $\mathrm{WO}_{3}$-monohydrate $\left(\mathrm{WO}_{3} \cdot \mathrm{H}_{2} \mathrm{O}\right)$, and

(iii) $\mathrm{WO}_{3}$-hemihydrate $\left(\mathrm{WO}_{3} \cdot 1 / 2 \mathrm{H}_{2} \mathrm{O}\right) / \mathrm{WO}_{3} \cdot 0.33 \mathrm{H}_{2} \mathrm{O}$.

$\mathrm{WO}_{3} \cdot 2 \mathrm{H}_{2} \mathrm{O}$ has a stratified layered structure composed of a $\mathrm{WO}_{5}(\mathrm{OH})_{2}$ layer sharing corners and the other water molecule is found in between the layers. Hence, it can be facially exfoliated into thin $2 \mathrm{D}$ nanosheets. ${ }^{81,159} \mathrm{WO}_{3} \cdot \mathrm{H}_{2} \mathrm{O}$ is composed of highly distorted $\mathrm{WO}_{5}(\mathrm{OH})_{2}$ units sharing corners and is coordinated by five oxygen atoms and one $\mathrm{H}_{2} \mathrm{O}$ molecule. As shown in Fig. 3e, it is believed that the crystal structure of $\mathrm{WO}_{3}$. $1 / 2 \mathrm{H}_{2} \mathrm{O}$ resembles a cubic pyrochlore type, where the water molecules are present in its tunnels. ${ }^{160}$ The inter-layer expansion can significantly reduce the ion diffusion energy barrier, ultimately improving the ion diffusion kinetics. Furthermore, the structural water molecules confined in the nano-interlayer spaces facilitate efficient proton transfer and induce a batteryto-pseudocapacitor transition in electrode materials. ${ }^{161,162}$ The interlayer water molecules can improve the intercalation capability of EC ions, which is significantly important in attaining superb NIR electrochromism in EC devices. Accordingly, Wang et al. ${ }^{163}$ demonstrated hydrated $\mathrm{WO}_{3}$ and studied the influence of water molecules in decreasing the energy barrier of ion diffusion and improving the ionic flux in the $\mathrm{WO}_{3}$ crystals. They found that $\mathrm{WO}_{3} \cdot 2 \mathrm{H}_{2} \mathrm{O}$ exhibited a good NIR extension with a large transmittance modulation (90.4\%), high coloration 
efficiency (322.6 $\mathrm{cm}^{2} \mathrm{C}^{-1}$ ), and high cyclic stability (maintaining $93.7 \%$ after 500 cycles), with good solar heat maintenance capability. $\mathrm{Li}$ and coworkers ${ }^{164}$ reported an EC hybrid material using $\mathrm{WO}_{3} \cdot \mathrm{H}_{2} \mathrm{O}$ nanosheets and a Persian-white film on FTO substrate as an asymmetric electrode. The film had an optical modulation of $61.7 \%$, response speed of $1.84 / 1.95 \mathrm{~s}$, coloration efficiency of $139.4 \mathrm{~cm}^{2} \mathrm{C}^{-1}$, and cyclic stability of $82 \%$.

The optical properties of $\mathrm{WO}_{3-x}$ can be measured by absorbing photon energy, which excites electrons from the valence band (occupied state) to the conduction band (empty state) and generates electron-hole pairs. ${ }^{169}$ The absorption coefficient $(\alpha)$ evaluated from the spectral transmittance, $T(\lambda)$, and reflectance, $R(\lambda)$, is given as eqn (1).

$$
\alpha(\lambda)=1 / d \ln [(1-R(\lambda)) /(T(\lambda))]
$$

Tungsten oxide is transparent in most visible wavelengths when the photon energy is lower than the bandgap energy. However, it shows a yellowish and blue color with a smaller bandgap, representing that some parts of blue light are absorbed. ${ }^{170,171}$ The optical absorption at a lower energy than the bandgap is because of the electronic transition between the localized states. ${ }^{172}$ However, when the photon energy is greater than the bandgap energy, the absorption can be described as follows:

$$
\varepsilon \propto \infty\left(\varepsilon-E_{\mathrm{g}}\right)^{\eta}
$$

where " $\varepsilon$ " is the photon energy and " $\eta$ " depends on the type of optical transition, for example, if $\eta=2$ it is an indirect and allowed transition.

Because of the reduction in particle size (quantum confinement effect), the bandgap becomes wide, resulting in light emission at shorter wavelengths. ${ }^{173}$ Nonetheless, the structural defects and oxygen vacancies in $\mathrm{WO}_{3-x}$ narrow the bandgap due to the defective band below the conduction band. The high concentration of oxygen vacancies in $\mathrm{WO}_{3-x}$ extend the defective band into the conduction band. This forms a degenerate semiconductor, which shifts the Fermi level upward, achieving metal-like conductivity, and thus highly improves the electrochromic, electrochemical, photocatalytic, and photochromic properties. ${ }^{171}$ The color of $\mathrm{WO}_{3}$ films changes from lightyellow to light-greenish and $\mathrm{WO}_{3-x}$ changes to dark blue, which shows a blue shift. Broad absorption bands appear in the red to NIR region and blue-green region. The absorption bands at these specific wavelengths make $\mathrm{WO}_{2.72}$ light green, where the additional absorption bands are due to the transfer of electrons from $\mathrm{W}^{6+}$ to $\mathrm{W}^{5+} \cdot{ }^{172}$ The excess electrons can be localized, which are known as small polarons, or transferred to adjacent W ions. ${ }^{174}$ The intercalated electrons may occupy the empty d-bands and develop a new electronic transition that is delocalized on crystalline $\mathrm{WO}_{3}$ or localize on the metal sites in an amorphous form and perform polaronic absorption. Amorphous $\mathrm{WO}_{3-x}$ exhibits a disordered structure, indicating the presence of broken bonds with variable bond lengths, bond angles, and coordination. In $\mathrm{WO}_{3-x}$, the localized states are found closer to the oxygen vacancies or tungsten atom, and thus the electrons and/or charge compensating ions can easily jump within the localized states. ${ }^{136,169,175}$

\subsection{Electrochromic mechanism}

The EC properties mainly depend on the particle size, dimensions, aspect ratio, crystallinity, porosity, thickness, and reversibility of films, together with their capability to accommodate guest ions $\left(\mathrm{H}^{+}, \mathrm{Na}^{+}\right.$, and $\left.\mathrm{Li}^{+}\right) \cdot{ }^{13,88,176}$ The coloration mechanism depends on the nature and behaviors of materials. In crystalline tungsten oxide films, their coloration is attributed to the Drude-like absorption of delocalized/free electrons, similar to the behavior of heavily doped metal oxides with ionized impurities. ${ }^{177}$ In amorphous tungsten oxide films, the optical absorption is caused by the electron exchange/polaron-topolaron transition between adjacent $\mathrm{W}$ atoms $\left(\mathrm{W}^{6+}, \mathrm{W}^{5+}\right.$, and $\mathrm{W}^{4+}$ states), and the inserted electrons are localized in the $\mathrm{W}^{5+}$ sites and polarize their surrounding lattice to form small polarons. ${ }^{178}$ Moreover, compared to crystalline films, amorphous $\mathrm{WO}_{3}$ films show a better coloration efficiency, which increases with an increase in oxygen vacancies. ${ }^{179}$ However, amorphous tungsten oxide films show poor stability because of their irregular atomic distribution. ${ }^{88}$

$\mathrm{W}_{18} \mathrm{O}_{49}$ is a promising EC smart material that changes color from clear to blue, dark-blue, and transparent with a faradaic charge. When protons/lithium ions intercalate and externally powered electrons are inserted into the tungsten oxide film, the transmissivity of the electrodes drops from $91.3 \%$ to $15.1 \%$ during charging. ${ }^{129,180}$ Two types of redox reactions occur upon voltage bias as follows: ${ }^{181}$ (a) a charge transfer process at the material-electrolyte interface, i.e., surface-dependent process and (b) diffusion controlled redox reactions within the material lattices, i.e., ion-diffusion dependent process. The intercalated cations exist in the channels formed by the $\mathrm{WO}_{6}$ octahedra or react with the bridging oxygen of the host ${ }^{156}$ by breaking the $\mathrm{W}-\mathrm{O}-\mathrm{W}$ networks and generating new $\mathrm{W}=\mathrm{O}$ bonds. ${ }^{128,182}$ Thus, the coloration (tungsten bronzes, $\mathrm{H}_{x} \mathrm{WO}_{3} / \mathrm{Li}_{x} \mathrm{WO}_{3}$ formation) is due to the reaction of cations with $\mathrm{W}=\mathrm{O}$ groups by forming colored $\mathrm{W}^{5+}-\mathrm{OH}$ states. ${ }^{181}$ It is assumed that cations can react with the bridging oxygen at a low concentration of inserted charges. However, at a high concentration, they can also react with $\mathrm{W}-\mathrm{O} .{ }^{128,181,183}$ Raman spectroscopy results show that the interaction between oxygen vacancies and $\mathrm{Li}^{+}$ions improves the coloration efficiency of $\mathrm{WO}_{2.72}$ films. ${ }^{129,136,179}$ When the applied voltage polarity is reversed, the $\mathrm{Li}^{+}$ions deintercalate and the film is bleached. These redox dynamics demonstrate that tungsten oxide can be transformed from a transparent state to an absorbent state to store electric energy.

The ion insertion and extraction in $\mathrm{WO}_{3}$ films can be shown as eqn (3)-(5):

Cathodic reaction,

$$
\begin{gathered}
{\left[\mathrm{WO}_{3-x}+\mathrm{H}^{+}+\mathrm{Xe}^{-}\right]_{\text {bleached }} \rightarrow\left[\mathrm{H}_{x} \mathrm{WO}_{3-x}\right]_{\text {colored }}} \\
{\left[\mathrm{WO}_{3-x}+\mathrm{Li}^{+}+\mathrm{Xe}^{-}\right]_{\text {bleached }} \rightarrow\left[\mathrm{Li}_{x} \mathrm{WO}_{3-x}\right]_{\text {colored }}}
\end{gathered}
$$

Anodic reaction 


$$
\left[\mathrm{Ni}(\mathrm{OH})_{2}\right]_{\text {bleached }} \rightarrow\left[\mathrm{NiOOH}+\mathrm{H}^{+}+\mathrm{e}^{-}\right]_{\text {colored }}
$$

Protic electrolytes are used for $\mathrm{H}^{+}$intercalation. Similarly, $\mathrm{Li}^{+}$ electrolytes are widely used in $\mathrm{WO}_{2.72}$ EC devices (Fig. 1). ${ }^{156}$ The colored state should be $\mathrm{H}_{x} \mathrm{WO}_{3}$ or $\mathrm{Li}_{x} \mathrm{WO}_{3}$, whereas some reactions reach $X<0.5$ states, resulting in complete reversibility. The hybridization in the octahedral geometry can be shown between the oxygen $2 \mathrm{p}$ orbital (valence band) separated from the tungsten $5 \mathrm{~d}$ orbitals (conduction band) with a bandgap (Fig. 4a and b). The d-orbital of tungsten in $\mathrm{WO}_{3}$ splits into two sub-orbitals, conventionally represented by "eg" \& "t2g",184 as given in Fig. $4 \mathrm{a}$ and b, respectively. In stoichiometric $\mathrm{WO}_{3}$, the "O2p" orbital is filled and the W5d orbital is empty (Fig. 4a), where the bandgap is wide enough, which results in glowing transmission in the thin films. The intercalation of external ions and the charge compensating electrons partially fill the empty d-orbitals, resulting in optical absorption. ${ }^{185}$

The removal of the oxygen atom (oxygen vacancy) from the $\mathrm{WO}_{3}$ structure produces two additional electrons that can be paired in a single spin state (closed-shell) (Fig. 4b) or an unpaired triplet spin state. Furthermore, single/two electrons can be transferred to nearby $\mathrm{W}$ atoms, resulting in $\mathrm{W}^{5+}$ and $\mathrm{W}^{5+}-\mathrm{W}^{5+}$ centers, respectively. The formation of an oxygen vacancy changes the $\mathrm{W}-\mathrm{W}$ bond distance across the $\mathrm{O}$ vacancy sites. Thus, the $\mathrm{W}-\mathrm{O}$ bond length and bond angles in the crystal change as the bonded orbitals split, leading to the hybridization of the W5d and $\mathrm{O} 2 \mathrm{p}$ orbitals. The inserted electrons and ions diffuse and localize on the tungsten atoms, reducing some of the $\mathrm{W}^{6+}$ to $\mathrm{W}^{5+}$ states. Because of the absorption of photons, the inserted electrons gain sufficient energy, which can be transferred to the adjacent sites ${ }^{184}$ or oxygen vacancies, indicating that oxygen vacancies have a significant impact on both the photochromic and EC properties. $^{130,131}$ The $\mathrm{W}^{4+}$ state is considered energetically unfavorable, although XPS results prove the existence of $\mathrm{W}^{4+}$ ions. ${ }^{136,186}$ However, XPS is surface sensitive and is not consistent about the bulk state of different tungsten oxide films. ${ }^{186}$ The most favorable condition is the creation of a double charge vacancy and two $\mathrm{W}^{5+}$ ions. The transfer of charges between two sites is represented by " $i$ " \& " $j$ ", as given in eqn (6).

$$
\mathrm{W} i^{5+}+\mathrm{Wj}^{6+}+\text { photon } \rightarrow \mathrm{Wi}^{6+}+\mathrm{Wj}^{5+}
$$

It is believed that the intercalated electrons diffuse in the localized state near the conduction band and the surrounding atoms move, and thus a potential well is generated. The obvious interaction of the electrons and phonons results in the formation of polarons. An electron interacting with a cloud of phonons is called polarons. ${ }^{187}$ The polaron-induced optical absorption in $\mathrm{W}_{18} \mathrm{O}_{49}$ produces a broadband absorption spectrum, making the film dark blue. ${ }^{184}$ Similarly, in the polaron-induced coloration, ${ }^{188}$ the electrons produced due to the oxygen vacancies are trapped in the $5 \mathrm{~d}$ orbitals of $\mathrm{W}_{18} \mathrm{O}_{49}$ (or defect sites) and then polarize the surrounding lattice to produce small polarons. The resulting polarons then simultaneously yield polaron-to-polaron transitions between two adjacent non-equivalent tungsten sites. In contrast, localized surface plasmons are the mutual oscillations of free electrons/ conductive electrons in materials with large carriers. ${ }^{135}$ Therefore, NIR absorption is widely interpreted as the result of the small polarons hopping and/or local surface plasmon resonance (LSPR). ${ }^{88}$

\section{Synthesis and film fabrication strategies of $\mathrm{WO}_{3-x} / \mathrm{WO}_{3}$}

Substantial efforts have been dedicated to synthesizing $\mathrm{WO}_{3}$ nanostructures with different morphologies, sizes, and dimensions (0D, 1D, 2D, and 3D), as displayed in Fig. 5. To date, tungsten oxide particles have been synthesized by varying the synthetic conditions and technologies, which greatly affect their size, morphology, and functional applications. According to the relevant reaction media, the techniques can be grouped into two main categories, i.e., gas-phase technologies and wetchemical synthesis methods. Gas-phase technology is classified as plasma sputtering, ${ }^{190}$ chemical vapor deposition, ${ }^{191}$ and physical vapor deposition. Wet-chemical synthesis includes Langmuir-Blodgett deposition, ${ }^{192}$ electrophoretic deposition, ${ }^{193}$ electrospinning, ${ }^{194}$ and hydrothermal ${ }^{195}$ and solvothermal synthesis. Similarly, spray-coating, inkjet printing, spin-coating, roll coating, screen printing, and layer-bylayer self-assembly have also been employed. ${ }^{196}$ However, the material synthesis and film fabrication processes are still challenging to adopt on a large scale. These technologies will be reviewed in this section.

\subsection{Gas-phase deposition methods}

4.1.1. Chemical vapor deposition (CVD). CVD is a common industrial technology used for depositing extremely highquality thin films on a variety of substrates. The commonly used CVD methods include atmospheric pressure chemical vapor deposition (APCVD) and aerosol-assisted chemical vapor deposition (AACVD). APCVD has certain merits, such as large area scalability, uniformity in thickness, and possibly low cost. For example, the APCVD process is used to manufacture largearea energy efficient FTO/ITO coatings for windows over three meters. ${ }^{199}$ The working principle of both the CVD and PVD methods can be found in the review published by Jiang et al. ${ }^{23}$ Blackman and Parkin ${ }^{200}$ used the APCVD method to deposit $\mathrm{WCl}_{6}$ dissolved in different solvents (ethanoic anhydride,

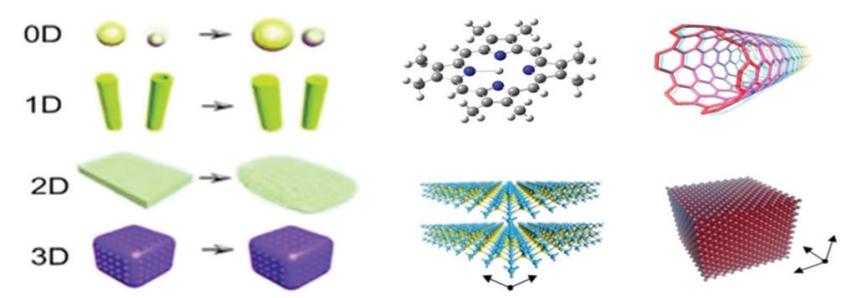

Fig. 5 Schematic illustration of the design of $\mathrm{WO}_{3-x} / \mathrm{WO}_{3}$ nanostructures with different shapes/dimensions. ${ }^{197}$ OD nanoparticles, 1D nanowires and nanotubes, 2D thin layers, and 3D porous nanostructure. Reproduced with permission. ${ }^{198}$ Copyright $(02019$, ACS. 
ethanoic acid, ethyl ethanoate, methanol, ethanol, 2-propanol, 2-methyl-2-propanol, and water) on a glass substrate. The thin films crystallized with ethanoic anhydride, water, and ethanoic acid were fully oxidized to a monoclinic phase of $\mathrm{WO}_{3}$ at $625{ }^{\circ} \mathrm{C}$. The deposited films revealed different surface morphologies, i.e., cubic to platelets, under similar conditions, which displayed significantly different performances in different applications, such as photocatalysis, gas sensors, and photochromism.

Brescacin et al. ${ }^{201}$ developed phosphorus (P)-doped amorphous $\mathrm{WO}_{3}$ films via CVD processes using volatile, low-melting P-doped tungsten carbonyls. The P-doping preserved the amorphous nature of the material and deposited film, even upon calcination at high temperatures. The tiny amount of dopant released by the precursor during its decomposition was sufficient to inhibit the crystallization of tungsten oxide on the matrix. The films deposited on common glass displayed remarkable EC performances with the maximum coloration efficiency of $66 \mathrm{~cm}^{2} \mathrm{C}^{-1}$. Ou et al. ${ }^{202}$ synthesized tungsten(vi) oxo-complexes of the type $\mathrm{WO}(\mathrm{OR})_{3} \mathrm{~L}$ having partially fluorinated alkoxide and/or chelating ligands (Fig. 6). The thermal decomposition behavior and mass spectrometry (MS) of selected examples were studied. With $\mathrm{WO}\left(\mathrm{OC}\left(\mathrm{CF}_{3}\right)_{2} \mathrm{CH}_{3}\right)_{3}$ as a single-source precursor, tungsten oxide $\left(\mathrm{WO}_{x}\right)$ was deposited via CVD and AACVD and compared. The obtained deposited coatings exhibited different crystallinity, structures, and morphology (nanowires, nanoplates, and nanocubes). These variations in morphology demonstrated that the solvent used during AACVD controls the growth of the film. The obtained results suggested that the materials deposited by both deposition methods allow not only the procedure to be scaled up, but also have the potential to generate the formation of a film on a wide area with EC properties.

Studies emphasized that $\mathrm{MoO}_{3}$ and $\mathrm{WO}_{3}$ have similar EC properties, and thus the APCVD method can be used solely to obtain a film composed of either Mo-doped $\mathrm{WO}_{3}$ or mixed MoW oxides via the pyrolytic decomposition of metal carbonyls. ${ }^{205}$ The visible light transmission of the film deposited on ordinary glass was about $80 \%$, while that on conductive glass (e.g., $\mathrm{SnO}_{2}: \mathrm{Sb} /$ glass) was about $60 \%$. It is also found that after annealing, the optical transmission of the single oxide films improved, while that of the mixed oxide films slightly decreased. The mixed oxide showed deep-blue coloring only at the cathode, minimizing the visible-light transmittance. Similarly, excellent reversibility with an optical modulation of about $30 \%$ was obtained. Numerous reports based on mixed Mo-W oxides have been published. ${ }^{206,207}$

4.1.2. Physical vapor deposition (PVD). PVD is another gasbased coating process, which involves metal transfer at the atomic level under vacuum conditions. This method may be related to $\mathrm{CVD}$, except that in $\mathrm{PVD}$, the depositing material is
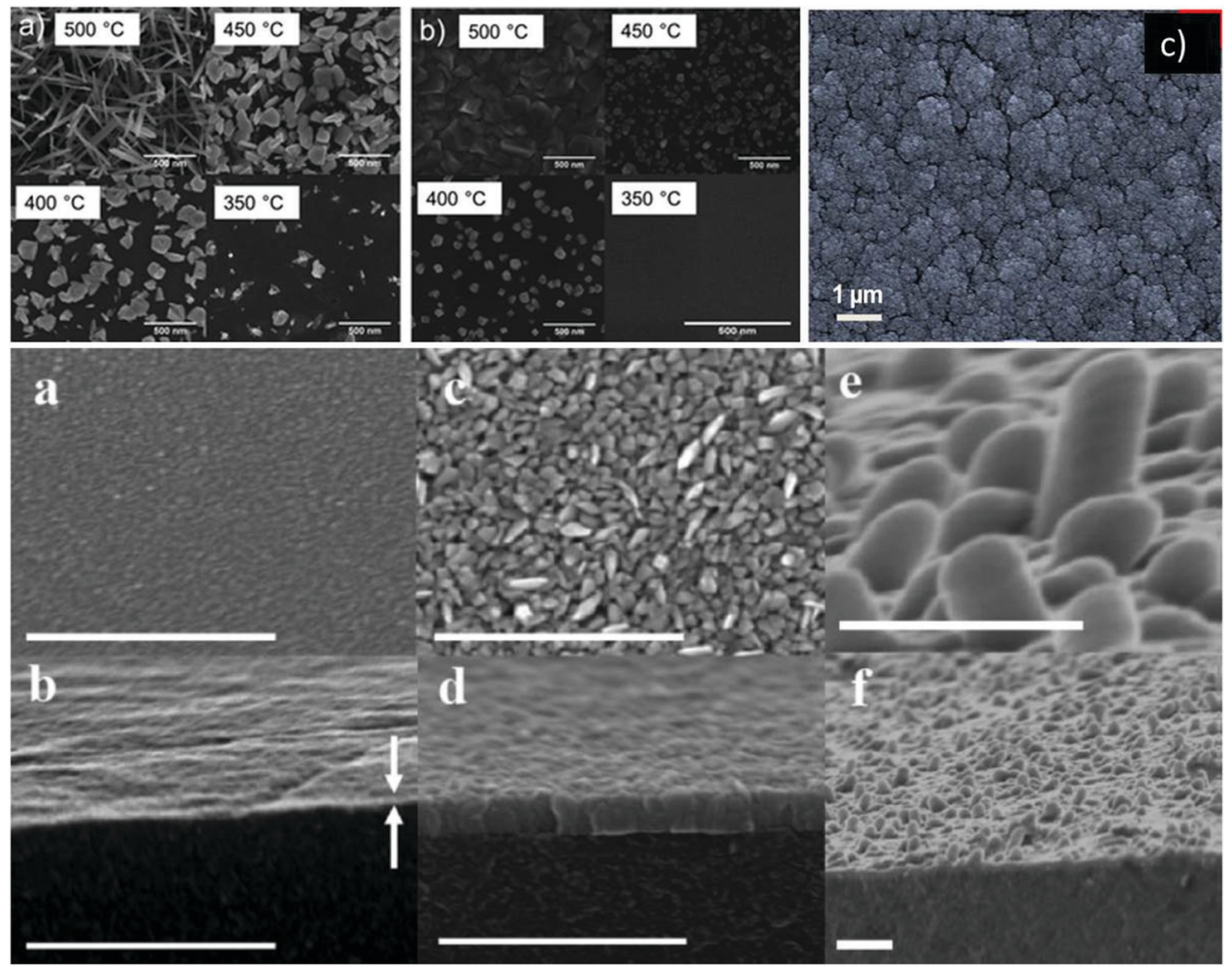

Fig. 6 (top) Plane-view SEM images of deposits grown on $\mathrm{Si}\langle 100\rangle$ at $500{ }^{\circ} \mathrm{C}, 450{ }^{\circ} \mathrm{C}, 400{ }^{\circ} \mathrm{C}$, and $350{ }^{\circ} \mathrm{C}$ from the precursor via (a) AACVD and (b) CVD.

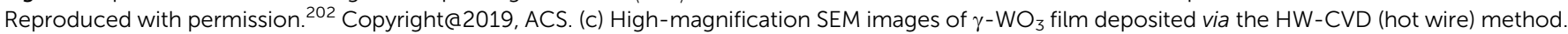
Reproduced with permission. ${ }^{203}$ Copyright(a2017, Wiley-VCH. (bottom) Deposition at different times. Top view (a) over 5 min, (c) over 60 min, and (e) over 60 min with $48 \mathrm{~h}$ of additional substrate heating during and after deposition. (b, $\mathrm{d}$ and f) View of fractured cross section of the corresponding films. All scale bars represent $500 \mathrm{~nm}$. Reproduced with permission. ${ }^{204}$ Copyright@2010, Wiley-VCH. 
initially in the solid state, whereas in CVD, the gaseous form of the precursor material is introduced into the reaction chamber.

PVD is comprised of four steps as follows:

(i) evaporation of materials deposited by high energy sources (e.g., electron beam/ions that evaporate atoms from the surface);

(ii) transport of the vapor to the substrate to be coated;

(iii) the reaction between the metal atoms and the appropriate reactive gas (such as oxygen, nitrogen, and methane) during the transport stage; and

(iv) deposition of the coatings on the surface of the substrate.

The PVD method has several advantages including (i) PVD thin coatings demonstrate better properties compared to the substrate materials; (ii) all types of inorganic materials and some types of organic materials can be used; and (iii) the procedure is more environmentally benign than several other techniques, e.g., simple electroplating. Meanwhile, the PVD method also has certain disadvantages, including (i) problems with coating complex shapes; (ii) high cost and low output; and (iii) complexity of the process.

Zhang et al. ${ }^{208}$ demonstrated the growth of a $\mathrm{WO}_{3}-\mathrm{WS}_{2}$ bilayer hybrid with $\mathrm{WO}_{3}$ monolayer species attached on the surface of large-size $\mathrm{WS}_{2}$ monolayers, as shown in Fig. 7a-d. The optical analysis showed that the photoluminescence quantum yield (PLQY) of the as-prepared $\mathrm{WO}_{3}-\mathrm{WS}_{2}$ hybrid was excellent and could reach $11.6 \%$ higher PL than that of the bare $\mathrm{WS}_{2}$ monolayers, which is about 13-times greater than that via the mechanical exfoliation of $\mathrm{WS}_{2}$ (Fig. 7e-g). The improved PL enhancement mechanism can be investigated by carrying out time-dependent optical measurements. The generation of $\mathrm{WO}_{3}-\mathrm{WS}_{2}$ hybrid structures with ultrahigh PLQY can provide an efficient method for developing highly effective $2 \mathrm{D}$ integrated photonic applications. Hadifakoor et al. ${ }^{209}$ fabricated a

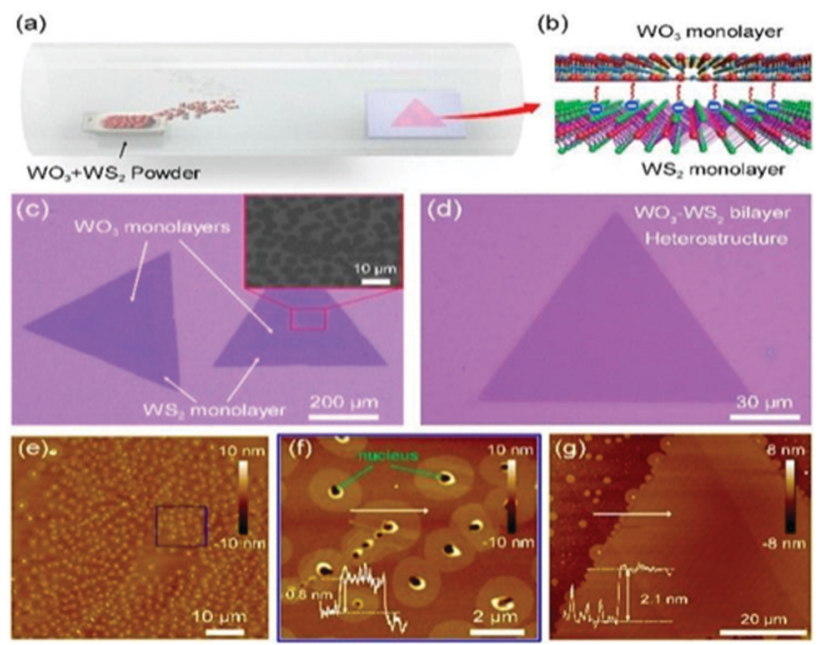

Fig. 7 ( $a$ and $b)$ Schematic of the synthetic process and 3D representation of the $\mathrm{WO}_{3}-\mathrm{WS}_{2}$ heterostructure. (c and d) Typical optical image of (c) partially covered and (d) completely covered $\mathrm{WO}_{3}-\mathrm{WS}_{2}$ heterostructures. The inset in (c) shows the SEM image of the area within the red box. $(e-g)$ AFM images of ( $e$ and $f$ ) partially covered and $(g)$ completely covered heterostructures. The insets show the corresponding height profile. Reproduced with permission. ${ }^{208}$ Copyright@2019, ACS.
DMD skeleton of $\mathrm{WO}_{3} / \mathrm{Ag} / \mathrm{WO}_{3}$ thin films with various thicknesses through the PVD approach via electron-beam evaporation under the vacuum at pressure of $10^{-5}$ torr and calcination temperature of $100-400{ }^{\circ} \mathrm{C}$. The structural and optical properties of the nano-multilayer composites structures were studied in heat mirror applications. Nanosized Ag layers with thicknesses of $10 \mathrm{~nm}, 12 \mathrm{~nm}$, and $14 \mathrm{~nm}$ were deposited on monolayer $\mathrm{WO}_{3}$ films. Atomic force microscopy (AFM) showed the morphology of the individual layers, indicating that a smoother layer can be achieved after annealing at $300{ }^{\circ} \mathrm{C}$. Ellipsometry analysis was performed to examine the bilayers, thickness of the Ag film layer, and inter-connectivity between the $\mathrm{WO}_{3}-\mathrm{Ag}-\mathrm{WO}_{3}$ layers. There was almost no interference among the $\mathrm{WO}_{3}-\mathrm{WO}_{3}$ layers in the samples with $\mathrm{Ag}$ thicknesses of $12 \mathrm{~nm}$ and $14 \mathrm{~nm}$. The efficiency of the heat mirrors was evaluated according to their optical characteristics and best performance. Recently, Hoseinzadeh et al. ${ }^{209}$ studied a $\mathrm{WO}_{3}$ and Ag composite deposited via the layer-by-layer (LbL) PVD method as an EC electrode on an FTO glass substrate. Nanosized silver noble metal can behave as an electron-trap center, which enables the departure of charges. The electrochemical and optical properties were examined through CV and UVvisible absorption experiments, which showed that heating the deposited film at $200{ }^{\circ} \mathrm{C}$ can provide much better conductivity $(90 \mathrm{~mA})$ and transmittance change $(\Delta T=90 \%$ at a continuous switching step), endowing $\mathrm{WO}_{3}-\mathrm{Ag}-\mathrm{WO}_{3}-\mathrm{Ag}$ with excellent EC properties. Esmail et al. ${ }^{210}$ demonstrated amorphous $\mathrm{WO}_{3}$ smooth films thinner than $200 \mathrm{~nm}$, which exhibited an excellent coloration efficiency with a switching speed in the order of seconds.

\subsection{Wet-chemical synthesis methods}

The gas-phase deposition of coatings is a highly pure and efficient route for the fabrication of $\mathrm{WO}_{3}$ films. However, the required instrumentation is very complex and expensive. Conversely, solution-based particle synthesis and film formation are cost effective, controllable, and high yielding, which provide an alternative reliable route for the production of smart coatings, reflective displays, and sensors. ${ }^{211}$

4.2.1. Sol-gel method. The "sol-gel" terminology describes a wide-range process, which is the production of a solid phase through the colloidal dispersion/gelation of a colloidal suspension (the sol). Further reactions lead to bonds between the sol particles, creating an infinite network of particles (gels). The "dry gel" (xerogel) state results after drying the gel, and subsequent heating can remove the unreacted organic impurities, stabilize the gel, densify, and crystalize the material. ${ }^{80,212}$ Thus, a metal solution can be converted to an oxide chain-like complex compound such as a metal alkoxide via a simple polymerization reaction and/or using a template(s). The compounds used for the oxide polymerization include common organic solvents, organic acids, and templates, and the polymerization occurs through hydrolysis/condensation. The sol-gel method was used only to prepare metals and semi-metal oxides, and then extended to organic-inorganic hybrids and occasionally for the production of pure organic 
networks. ${ }^{80} \mathrm{WO}_{3}, \mathrm{~V}_{2} \mathrm{O}_{5}, \mathrm{MnO}_{2}$, and many other transition-metal oxides display numerous oxidation states. The composition and structure of their precursors are susceptible to the $\mathrm{pH}$ and redox states, and hence they can control the sol and gelation. ${ }^{80}$ The sol-gel deposition techniques include dip-coating, ${ }^{213-216}$ spin-coating, ${ }^{217}$ inkjet-printing, ${ }^{218,219}$ Langmuir-Blodgett deposition, ${ }^{220-222}$ and spray-pyrolysis. ${ }^{76,223}$ This technology requires cheaper equipment for large-area surface coatings compared to gas-phase technologies.

The sol-gel method is attractive due to the following reasons:

(i) it provides an easy route for the preparation of metal oxide microstructures and mixed oxides with a controlled composition and homogeneity,

(ii) processing produces an amorphous hydrogel, which is better than crystalline films due to its excellent elasticity,

(iii) can reform volume changes caused by redox reactions, and improve conductivity,

(iv) films can well adhere to ITO/FTO films and be processed at ambient pressure, and (v) deals with a new deposition method of $\mathrm{WO}_{3}$-based coating on a large scale.

In 1984, Chemseddine et $a .^{224}$ first reported the results of a sol-gel $\mathrm{EC} \mathrm{WO}_{3}$ film, which was then subjected to different solgel processes for the production of pristine $\mathrm{WO}_{3}$ and mixed oxides. The coloration efficiency values compared to the reported sol-gel films were $70 \mathrm{~cm}^{2} \mathrm{C}^{-1}$ (at $685 \mathrm{~nm}$ ) and 223$167 \mathrm{~cm}^{2} \mathrm{C}^{-1}$ (at $\left.800 \mathrm{~nm}\right)^{225}$ compared to $115 \mathrm{~cm}^{2} \mathrm{C}^{-1}$ (at $633 \mathrm{~nm}$ ) for the amorphous films ${ }^{226}$ produced via thermal evaporation and $42 \mathrm{~cm}^{2} \mathrm{C}^{-1}$ (at $650 \mathrm{~nm}$ ) reported for sputtered polycrystalline films. ${ }^{227-229}$ The advantage of the sol-gel technique is its capability to generate various mixed oxides from a variety of hybrid mixtures with controlled compositions. ${ }^{230-240}$ Nguyen et al. ${ }^{241}$ used the sol-gel spin coating process and further annealing to form amorphous EC $\mathrm{WO}_{x}$ thin films. The precursor solutions were prepared from ammonium metatungstate hydrate $\left(\left(\mathrm{NH}_{4}\right)_{6}\left[\mathrm{H}_{2} \mathrm{~W}_{12} \mathrm{O}_{40}\right] \cdot 4 \mathrm{H}_{2} \mathrm{O}\right)$ and dimethylformamide (DMF) to produce a smooth and transparent amorphous $\mathrm{WO}_{x}$ film via spin-coating followed by calcination in air at $250{ }^{\circ} \mathrm{C}$ for $2 \mathrm{~h}$. The obtained EC film exhibited excellent
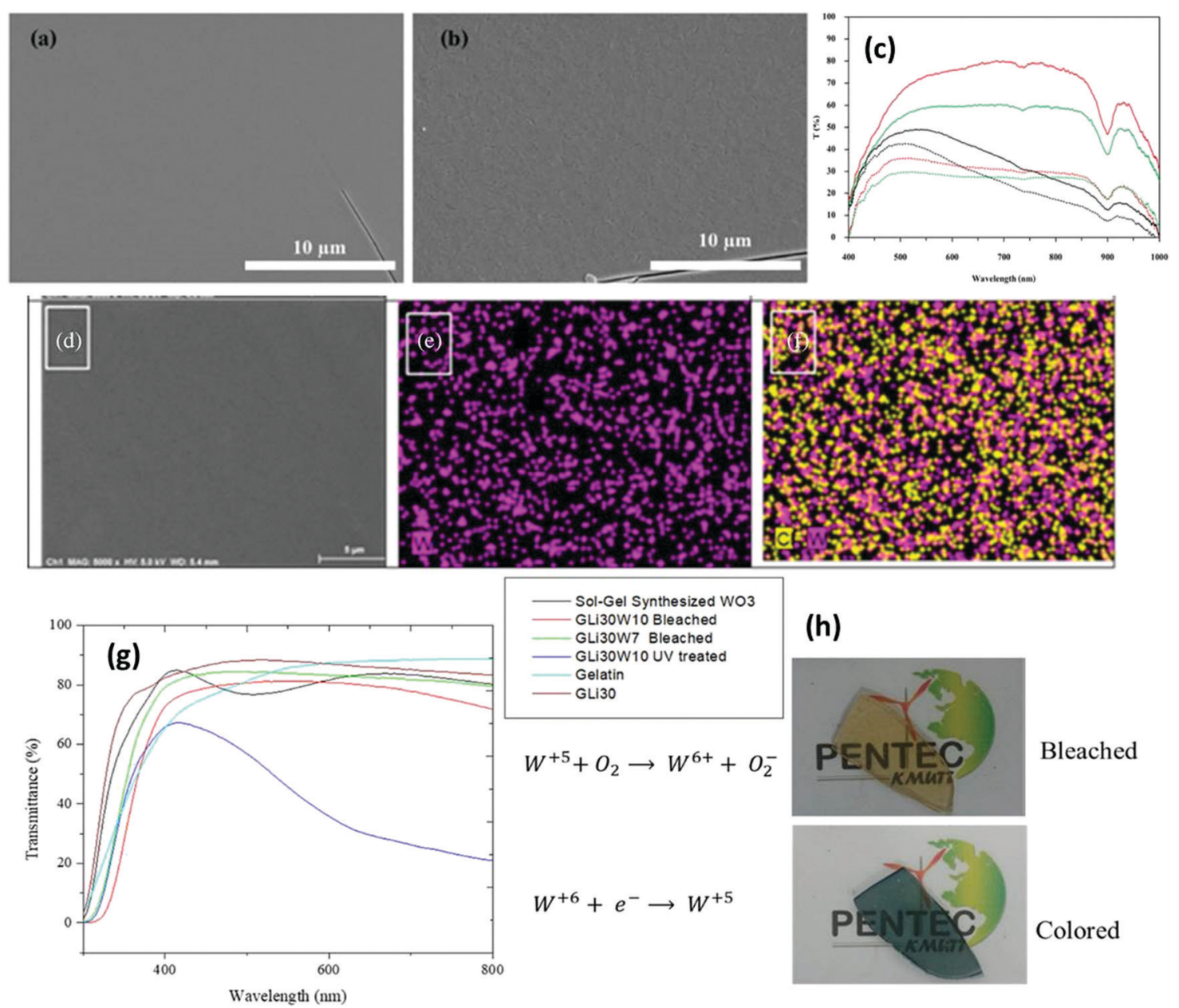

(h)

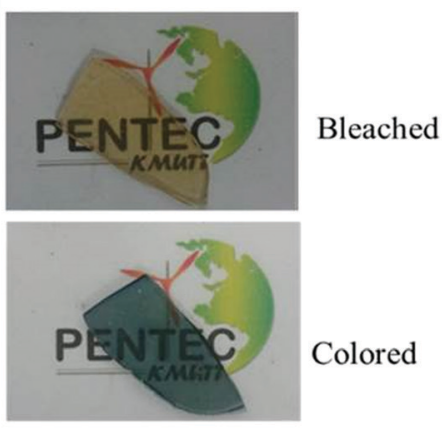

Fig. 8 Surface morphology of the $5 \% \mathrm{MoO}_{3}-95 \% \mathrm{WO}_{3}$ film annealed at (a) $300{ }^{\circ} \mathrm{C}$ and (b) $400{ }^{\circ} \mathrm{C}$. (c) Transmittance spectra. Reproduced with permission. ${ }^{243}$ Copyright@2017. Elsevier. (d) Film containing 30 wt\% $\mathrm{LiClO}_{4}$ and $10 \mathrm{wt} \% \mathrm{WO}_{3}$ (sample denoted as $\mathrm{GLi}_{30} \mathrm{~W}_{10}$ ), (e) tungsten map and (f) chlorine and tungsten distribution mapping. (g) Transmittance spectra of free-standing films and (g) optical images of colored and bleached states. Reproduced with permission. ${ }^{242}$ Copyright@2020, Wiley-VCH. 
stability over 4000 switching cycles with a coloration efficiency of $125 \mathrm{~cm}^{2} \mathrm{C}^{-1}$ and color/bleach times of 5/2.5 s. Amorphous $\mathrm{WO}_{x}$ film-based devices are impeccable for next-generation smart windows and other electronic display devices. Recently, Azarian et al. ${ }^{242}$ explored amorphous electro-photochromic films formed via the in situ sol-gel synthesis of $10 \mathrm{wt} \% \mathrm{WO}_{3}$ and $30 \mathrm{wt} \% \mathrm{LiClO}_{4}$ (denoted as $\mathrm{GLi}_{30} \mathrm{~W}_{10}$ ) in a gelatin/lithium cosolvent mixture (Fig. 8d-f). The transparent single-layer film was flexible and adhesive, active under UV radiation with a photo-response time of $30 \mathrm{~s}$, and gradually reversed once the UV source was blocked. Besides, in the presence of a conductive ion storage anode, the film on ITO glass exhibited an excellent EC response. The EC device configured with $\mathrm{ITO} / \mathrm{GLi}_{30} \mathrm{~W}_{10} / \mathrm{NiO} /$ ITO achieved a coloration efficiency of $51.54 \mathrm{~cm}^{2} \mathrm{C}^{-1}$ and fast color-bleach response times $(2 \mathrm{~s} / 1 \mathrm{~s})$. Jittiarporn et al. ${ }^{243}$ prepared hybrid $\mathrm{MoO}_{3}-\mathrm{WO}_{3}$ thin films on ITO glass via a sol-gel dip-coating process (Fig. 8). The formation of the film could be influenced by the calcination temperature, the concentration of $\mathrm{MoO}_{3}$ (0-10\%), and the copolymer template of Pluronic P-123 triblock $(0-20 \% \mathrm{w} / \mathrm{v})$. The lowest amount of $5 \% \mathrm{MoO}_{3}-95 \% \mathrm{WO}_{3}$ films without the use of the template and $200-300{ }^{\circ} \mathrm{C}$ annealing resulted in a high $\mathrm{CE}$, excellent cycling stability, and low response time. The surface roughness and low crystallinity of the films were thought to be attributed to their enhanced EC features. Amorphous mesoporous $\mathrm{WO}_{3}$ with a pore size in the range of 20-30 nm was fabricated via evaporation induced selfassembly and used as an electrochromic supercapacitor (ECS). ${ }^{120}$ The resultant device based on ECS exhibited excellent optical modulation ( $76 \%$ at $700 \mathrm{~nm})$, ultrafast switching speeds ( $0.8 \mathrm{~s}$ for coloration and $0.4 \mathrm{~s}$ for bleaching), and a high areal capacitance $\left(2.57 \mathrm{mF} \mathrm{cm}{ }^{-2}\right)$, even at a high current density (1.0 $\left.\mathrm{mA} \mathrm{cm}^{-2}\right)$.

Sun et $a .^{244}$ prepared mixed molybdenum-tungsten oxides with different stoichiometries $\left(\mathrm{Mo}_{x} \mathrm{~W}_{1-x} \mathrm{O}_{3}, 0<x<1\right)$ via solgel deposition from peroxo-polymolybdotungstate solutions onto FTO glass substrates. The $\mathrm{Mo}_{x} \mathrm{~W}_{1-x} \mathrm{O}_{3}$ films were annealed at $450{ }^{\circ} \mathrm{C}$. The transmittance modulation of the mixed oxide films was improved compared to the pristine $\mathrm{WO}_{3}$ film and no broadening of spectral modulation was observed. Kharade et $a .^{231}$ also employed a similar method to prepare $\mathrm{MoO}_{3}$ mixed $\mathrm{WO}_{3}$ thin films via hybrid physicochemical synthesis involving the microwave-assisted sol-gel preparation of $\mathrm{WO}_{3}$ and vacuum evaporation of $\mathrm{MoO}_{3}$ on an FTO glass substrate. Similarly, a mesoporous $\mathrm{WO}_{3}-\mathrm{TiO}_{2}$ composite film was fabricated via a sol-gel-based two-stage dip-coating technique on ITO glass, and subsequently annealed at $450{ }^{\circ} \mathrm{C}, 500{ }^{\circ} \mathrm{C}$, and $600{ }^{\circ} \mathrm{C} .{ }^{245}$ The composite film calcined at $600{ }^{\circ} \mathrm{C}$ contained orthorhombic $\mathrm{WO}_{3}$ and anatase $\mathrm{TiO}_{2}$. Mesoporosity was achieved in the film by employing silicates as templates. Mixed hexagonal and monoclinic phases were obtained upon calcination at $450{ }^{\circ} \mathrm{C}$ and $500{ }^{\circ} \mathrm{C}$. The results show that nanocrystal anatase $\mathrm{TiO}_{2}$ can stabilize the orthorhombic $\mathrm{WO}_{3}$ phase by calcination at $600{ }^{\circ} \mathrm{C}$. The mesoporous dye-sensitized $\mathrm{WO}_{3}-$ $\mathrm{TiO}_{2}$ composite film calcined at $600{ }^{\circ} \mathrm{C}$ showed an optical modulation of $51 \%$ in the NIR region. In contrast, the devices examined for the composite layers annealed at $450{ }^{\circ} \mathrm{C}$ and
$500{ }^{\circ} \mathrm{C}$ displayed a moderate optical modulation of $24.9 \%$ and $38 \%$, respectively.

4.2.2. Hydrothermal and solvothermal methods. The solvothermal and hydrothermal methods are commonly used for the synthesis of nanostructured materials. These methods allow the generation of various nanoscale morphologies and the possibility of structural control, which may not be afforded by high-temperature wet-chemical reactions. Hydrothermal synthesis involves the use of water-soluble precursor compounds at room temperature, which is cost-effective, and also depends on the relevant reaction variables, e.g., concentration, $\mathrm{pH}$, temperature, time, and reducing agents, that control the structural evolution, morphology, and size in the required dimensions (Table 2).

Due to its distinctive features, the hydrothermal method is considered the most suitable route for the synthesis of tungsten-based nanostructures. The solvothermal route involves nonaqueous media, which provide unique physicochemical characteristics to the material design for various hightech devices. Because of the wide applications of $\mathrm{WO}_{3}$ and $\mathrm{WO}_{3-x}$ nanostructures in energy-saving high-tech devices, many research groups have focused on these materials in the last few years. For example, Guo et al. ${ }^{277}$ prepared $\mathrm{W}_{18} \mathrm{O}_{49}$ nanorods by pyrolyzing the $\left(\mathrm{NH}_{4}\right)_{x} \mathrm{WO}_{3+x / 2}$ precursor, which was synthesized in the presence of an inorganic surfactant $\left(\left(\mathrm{NH}_{4}\right)_{2} \mathrm{SO}_{4}\right)$ in a reducing environment. Pan et al. ${ }^{260}$ used glycerol and ammonium sulfate $\left(\left(\mathrm{NH}_{4}\right)_{2} \mathrm{SO}_{4}\right)$ as a surfactant and capping agent, respectively, for the synthesis of coral-like nanostructures via the hydrothermal method. The obtained product showed excellent EC properties, including a fastswitching time (6/5 $\mathrm{s}$ for bleaching/coloration), a good $\mathrm{CE}$ $\left(109.6 \mathrm{~cm}^{2} \mathrm{C}^{-1}\right)$, and a large optical modulation $(\sim 78.1 \%)$ at $630 \mathrm{~nm}$ by applying a potential of \pm 1 V. Similarly, Kadam et $a .^{278}$ used propylene glycol (PG) as a surfactant to increase the size of the nanocrystals on the surface of ITO glass, which was then used for EC application. Chu et al. ${ }^{279}$ used a seed layer and a surfactant-free hydrothermal method to synthesize nanoplates on FTO glass. The obtained highly oriented 2D nanostructure exhibited potential for practical applications in smart windows due to its fast-switching speeds of $12 \mathrm{~s}$ and $3 \mathrm{~s}$ for coloration and bleaching, respectively. Mathuri et $a .^{280}$ also used an FTO glass substrate and synthesized $\mathrm{WO}_{3}$ nanoplates; however, they used different hydrothermal reaction times $(8-16 \mathrm{~h})$, and temperatures $\left(120-180{ }^{\circ} \mathrm{C}\right)$, which resulted in hexagonal and monoclinic $\mathrm{WO}_{3}$ nanoplates. The $\mathrm{WO}_{3}$ deposited films at $120{ }^{\circ} \mathrm{C}$ and $180{ }^{\circ} \mathrm{C}$ for different reaction times of $8 \mathrm{~h}, 12 \mathrm{~h}$, and $16 \mathrm{~h}$ possessed different surface morphologies, such as buds-like, bricks-like, sheets, and platelets-like structures. The optical bandgap of the deposited $\mathrm{WO}_{3}$ films varied in range of $2.5-2.9 \mathrm{eV}$ and $2.6-2.75 \mathrm{eV}$, respectively.

Liang et $a .^{281}$ produced a $\mathrm{WO}_{3}$ film using FTO-coated glass via the hydrothermal method. Their results showed that different $\mathrm{WO}_{3}$ nanoparticles such as nanosheets, nanoflakes, nanocuboids, and 3D nanowire flowers could be synthesized by adjusting the precursor solution composition. The solvent plays 
Table 2 List of the preparation methods and EC properties of OD, 1D, 2D, and 3D $\mathrm{WO}_{2.72} / \mathrm{WO}_{3}$-based nanoparticles

\begin{tabular}{|c|c|c|c|c|c|c|}
\hline Technology & Methods & Sub-method & Materials & Morphologies & $\begin{array}{l}\text { Coloration efficiency, } \\
\text { color/bleach response and } \\
\text { cyclic stability (cycles) }\end{array}$ & Ref. \\
\hline \multirow{2}{*}{$\begin{array}{l}\text { Mechanochemical } \\
\text { process (Ball } \\
\text { milling) }\end{array}$} & & & $\mathrm{Na}_{x} \mathrm{WO}_{3}$ (bronze) & Nanoparticles & & 246 \\
\hline & & $\begin{array}{l}\text { Magnetron } \\
\text { sputtering }\end{array}$ & $\mathrm{WO}_{x}$ & & $46.9 \mathrm{~cm}^{2} \mathrm{C}^{-1}, 7.8 / 117.8 \mathrm{~s}$ & 247 \\
\hline \multirow{5}{*}{$\begin{array}{l}\text { Gas-phase } \\
\text { technologies }\end{array}$} & CVD/PVD & R.F. sputtering & $\mathrm{WO}_{3}$ & & $46.45 \mathrm{~cm}^{2} \mathrm{C}^{-1}, 8.33 / 4.16 \mathrm{~s}$ & 248 \\
\hline & & R.F. sputtering & $\mathrm{WO}_{3+x}$ & Granular & $\sim 213 \mathrm{~cm}^{2} \mathrm{C}^{-1}, 5000$ & 249 \\
\hline & & $\begin{array}{l}\text { Thermal vapor } \\
\text { deposition }\end{array}$ & $\mathrm{WO}_{3-x}$ & Nanorods & & 250 \\
\hline & & HIPIMS & $\mathrm{WO}_{3} / \mathrm{Ag} / \mathrm{WO}_{3}$ & & $\begin{array}{l}30.5 \mathrm{~cm}^{2} \mathrm{C}^{-1}, 15 / 22.4 \mathrm{~s} \\
2500\end{array}$ & 125 \\
\hline & & APCVD & $\mathrm{WO}_{3-x}$ & $\begin{array}{l}\text { Nanowires, } \\
\text { nanoplates }\end{array}$ & & 200 \\
\hline \multirow[t]{31}{*}{$\begin{array}{l}\text { Wet-chemical } \\
\text { technologies }\end{array}$} & $\begin{array}{l}\text { Hydrothermal/ } \\
\text { solvothermal }\end{array}$ & & $\mathrm{WO}_{2.72}$ & Sub-micro fibers & & 251 \\
\hline & & & $\mathrm{WO}_{2.72}$ & Nanodots & $154 \mathrm{~cm}^{2} \mathrm{C}^{-1}, 1 / 1 \mathrm{~s}$ & $252-255$ \\
\hline & & & $\mathrm{WO}_{2.72}$ & Nanocrystals/particles & $\begin{array}{l}124.5 \mathrm{~cm}^{2} \mathrm{C}^{-1}, 1.9 / 2.8 \mathrm{~s} \\
1000\end{array}$ & $\begin{array}{l}139 \text { and } \\
256\end{array}$ \\
\hline & & & $\mathrm{WO}_{2.72}$ & Nanowires & $(-)$ & 135 \\
\hline & & & $\mathrm{WO}_{2.72}$ & Nanowires assembled & $35.7 \mathrm{~cm}^{2} \mathrm{C}^{-1}, 2 / 4 \mathrm{~s}, 1000$ & $\begin{array}{l}119 \text { and } \\
257\end{array}$ \\
\hline & & & $\mathrm{WO}_{3}$ & $\begin{array}{l}\text { Nanorods/Nanowires } \\
\text { arrays }\end{array}$ & $\begin{array}{l}102.8 \mathrm{~cm}^{2} \mathrm{C}^{-1}, 25.2 / 18 \mathrm{~s} \\
7.6 / 4.2 \mathrm{~s}, 3000\end{array}$ & $\begin{array}{l}229 \text { and } \\
258\end{array}$ \\
\hline & & & $\mathrm{WO}_{3}$ & Nanoparticles & $151.9 \mathrm{~cm}^{2} \mathrm{C}^{-1}, 1.3 / 1.7 \mathrm{~s}$ & 259 \\
\hline & & & & Nanofibers & $\begin{array}{l}109.6 \mathrm{~cm}^{2} \mathrm{C}^{-1}, 2.7 / 7.9 \mathrm{~s}, \\
4000\end{array}$ & 260 \\
\hline & & & $\mathrm{WO}_{3}$ & Particles & $\begin{array}{l}178.7 \mathrm{~cm}^{2} \mathrm{C}^{-1}, 1.9 / 2.5 \mathrm{~s} \text {, } \\
5000\end{array}$ & 261 \\
\hline & & & $\mathrm{WO}_{3}$ & Nanoflakes/sheets & $55.6 \mathrm{~cm}^{2} \mathrm{C}^{-1}, 5.2 / 2.2,3000$ & 262 \\
\hline & & & $\mathrm{WO}_{2.72}$ & Nanowires/Nanotrees & $75.35 \mathrm{~cm}^{2} \mathrm{C}^{-1+}, 2.64 / 7.28 \mathrm{~s}$ & 263 \\
\hline & & & $\mathrm{WO}_{2.72}$ & Nanoneedles & $150 \mathrm{~cm}^{2} \mathrm{C}^{-1}, 500$ & 264 \\
\hline & & & $\mathrm{WO}_{2.72}$ & Nanobelts & $(-)$ & 265 \\
\hline & & & $\mathrm{WO}_{3}$ & Nanobricks & $39.24 \mathrm{~cm}^{2} \mathrm{C}^{-1}, 9.7 / 6.9 \mathrm{~s}$ & 266 \\
\hline & & & $\mathrm{WO}_{3} / \mathrm{NiO}$ & $\begin{array}{l}\text { Nanoporous } \\
\text { structures }\end{array}$ & $85.9 \mathrm{~cm}^{2} \mathrm{C}^{-1}, 21.76 / 4.54 \mathrm{~s}$ & 267 \\
\hline & & & $\begin{array}{l}\mathrm{WO}_{3} \cdot \mathrm{H}_{2} \mathrm{O}, \mathrm{WO}_{3} . \\
2 \mathrm{H}_{2} \mathrm{O}\end{array}$ & Nanoplates & $\begin{array}{l}384.6 \mathrm{~cm}^{2} \mathrm{C}^{-1}, 322.6 \mathrm{~cm}^{2} \\
\mathrm{C}^{-1}, 500\end{array}$ & 163 \\
\hline & & & $\mathrm{WO}_{2.72} / \mathrm{POMs}$ & Nanowires & $42.31 \mathrm{~cm}^{2} \mathrm{C}^{-1}, 52 / 26 \mathrm{~s}, 500$ & 122 \\
\hline & & & $\mathrm{WO}_{3}-\mathrm{TiO}_{2}$ & $\begin{array}{l}\text { Hierarchical } \\
\text { nanostructure }\end{array}$ & $128.3 \mathrm{~cm}^{2} \mathrm{C}^{-1}, 6 \mathrm{~s}, 1000$ & 268 \\
\hline & & & $\mathrm{WO}_{x}-\mathrm{NbO}_{x}$ & Nanowires & & \\
\hline & & & $\mathrm{WO}_{2.72}$ & Mesoporous & $80.5 \mathrm{~cm}^{2} \mathrm{C}^{-1}, 5.3 / 3 \mathrm{~s}$ & 70 \\
\hline & & & $\mathrm{WO}_{2.72}$ & Mesoporous & $46.9 \mathrm{~cm}^{2} \mathrm{C}^{-1}, 7.8 / 117 \mathrm{~s}, 8$ & 247 \\
\hline & Sol-gel & Spin coating & $\mathrm{WO}_{x}$ & & $125 \mathrm{~cm}^{2} \mathrm{C}^{-1}, 5 / 2.5 \mathrm{~s}, 4000$ & 241 \\
\hline & & Dip coating & $\mathrm{WO}_{3}$ & Mesoporous & $35.8 \mathrm{~cm}^{2} \mathrm{C}^{-1}, 16 / 30 \mathrm{~s}$ & 269 \\
\hline & & Electrodeposition & $\mathrm{Ag}-\mathrm{WO}_{3}$ & Nanowires composite & $45.3 \mathrm{~cm}^{2} \mathrm{C}^{-1}, 12 / 2 \mathrm{~s}$ & 270 \\
\hline & & Inkjet printing & $\mathrm{WO}_{3} / \mathrm{TiO}_{2} / \mathrm{WO}_{X}$ & & $480 \mathrm{~cm}^{2} \mathrm{C}^{-1}, 3.3 / 2.8 \mathrm{~s}$ & 271 \\
\hline & & & $\mathrm{WO}_{3}$-PEDOT:PSS & composite & $\begin{array}{l}68.8 \mathrm{~cm}^{2} \mathrm{C}^{-1}, 2.4 / 0.8 \mathrm{~s} \\
10000\end{array}$ & 272 \\
\hline & & Langmuir-Blodgett & $\mathrm{WO}_{3}$ & Nanobundles & $41.8 \mathrm{~cm}^{2} \mathrm{C}^{-1}$ & 273 \\
\hline & & $\begin{array}{l}\text { Electrostatic force- } \\
\text { assisted printing } \\
\text { Spray pyrolysis }\end{array}$ & $\mathrm{WO}_{3}$ ink & $\mathrm{WO}_{3}$ powder & $\begin{array}{l}51.5 \mathrm{~cm}^{2} \mathrm{C}^{-1}, 65.5 \% \text { at } \\
700 \mathrm{~nm}, 25 \mathrm{~s} / 24 \mathrm{~s}\end{array}$ & 274 \\
\hline & & $\begin{array}{l}\text { Pulsed electro- } \\
\text { chemical deposition }\end{array}$ & $\mathrm{WO}_{3}$ & Poros films & $118.3 \mathrm{~cm}^{2} \mathrm{C}^{-1}, 6 / 2.7 \mathrm{~s}$ & 275 \\
\hline & & $\begin{array}{l}\text { Electrochemical } \\
\text { deposition }\end{array}$ & $\mathrm{WO}_{3}$ & $\begin{array}{l}\text { Nanorods/ } \\
\text { nanoparticles }\end{array}$ & $\sim 90 \mathrm{~cm}^{2} \mathrm{C}^{-1}, 3 / 2 \mathrm{~s}$ & 185 \\
\hline & & $\begin{array}{l}\text { Electrochemical } \\
\text { deposition }\end{array}$ & $\mathrm{WO}_{3}$ & Nanogranular & $41.16 \mathrm{~cm}^{2} \mathrm{C}^{-1}, 7.5 / 3.9 \mathrm{~s}$ & 276 \\
\hline
\end{tabular}

a significant role in controlling the shape and size of nano$\mathrm{WO}_{3}$. The transmission spectra indicated that the $\mathrm{WO}_{3}$ film showed $50 \%$ and $70 \%$ transmittance in the visible spectrum both in the colored and bleached state, respectively, which with the applied potential of $\pm 1.0 \mathrm{~V}$, was maintained for 1000 continuous coloration/bleaching cycles. Bhosale et al. ${ }^{261}$ used a wet-etching process and fabricated a $\mathrm{WO}_{3}$ EC film on ITO glass via the hydrothermal method. The etched $\mathrm{WO}_{3}$ exhibited 
roughness, porosity, and an open-tunnel structure. The resultant films showed excellent coloration efficiency $\left(\sim 178.7 \mathrm{~cm}^{2} \mathrm{C}^{-1}\right)$, a fast response time $(1.9 / 2.5 \mathrm{~s})$, transmittance modulation $(\sim 49 \%$ at $630 \mathrm{~nm}$ ), and stability (5000 cycles).

Lu et al. ${ }^{282}$ prepared uniform $\mathrm{WO}_{3}$ nanorods using $\mathrm{NaCl}$ as a capping agent. The solution $\mathrm{pH}$ and amount of $\mathrm{NaCl}$ played key roles in the morphology and microstructure evolution. The prepared nanorods were aligned on ITO glass via the dropcasting method. Sun et al. ${ }^{283}$ synthesized $\mathrm{W}_{18} \mathrm{O}_{49}$ nanowires via the solvothermal method using $\mathrm{WCl}_{6}$ and cyclohexanol precursors. The sample calcined up to $400{ }^{\circ} \mathrm{C}$ was stable and no phase change was observed; however, upon increasing the calcination temperature to $>400{ }^{\circ} \mathrm{C}$, a phase conversion to monoclinic $\mathrm{WO}_{3}$ occurred. Lu et al. ${ }^{284}$ grew a vertical nanowire array on FTO glass for the fabrication of an EC working electrode. They used $\mathrm{WCl}_{6}, \mathrm{CH}_{3} \mathrm{OH}$, and polyethylene glycol (PEG) as a capping agent. The $\mathrm{WO}_{3}$ nanowires coated FTO glass showed maximum coloration/bleaching of $41.63 \%$ at $632.8 \mathrm{~nm}$ and the switching times of $9.8 \mathrm{~s}$ and $2 \mathrm{~s}$, respectively. Li and coworkers $^{263}$ synthesized hierarchical nanotree-like $\mathrm{WO}_{3}$ architectures directly via seed-mediated growth on FTO, as shown in Fig. 9a-f. They used $\mathrm{H}_{2} \mathrm{WO}_{4}, \mathrm{SC}\left(\mathrm{NH}_{2}\right)_{2}, \mathrm{C}_{4} \mathrm{H}_{4} \mathrm{O}_{4}$, aqueous $\mathrm{HCl}$, and FTO glass and hydrothermal treatment to form a $\mathrm{WO}_{3}$ seed layer. The same precursors were used and the seed layer on FTO was put in an autoclave to grow the $\mathrm{WO}_{3}$ nanotree architecture. They reported that the nanotree trunks showed $\langle 002\rangle$ orientation growth, whereas the branches exhibited $\langle 100\rangle$ and $\langle 200\rangle$ oriented growth directions. The optical modulation was $74.7 \%$ at $630 \mathrm{~nm}$ at a potential $0.2 \mathrm{~V}$ (Fig. 9g). The coloration efficiency (Fig. 9h) was $75.35 \mathrm{~cm}^{2} \mathrm{C}^{-1}$ with fast switching responses of 2.64/7.28 s. The electrochemical and EC properties of the $\mathrm{WO}_{3}$ nanotrees were attributed to their large surface area, showing potential for application in EC smart windows.

Li et al. ${ }^{264}$ synthesized needle-like $\mathrm{W}_{18} \mathrm{O}_{49}$ to form a thin film on an ITO substrate. The needle-like $\mathrm{W}_{18} \mathrm{O}_{49}$ nanocrystals exhibited a good performance and control over NIR light. Their powder-based optical properties also showed that the needlelike $\mathrm{W}_{18} \mathrm{O}_{49}$ nanocrystals have excellent photoabsorption in the NIR region, corresponding to 780-2500 $\mathrm{nm}$. The LbL-coated film exhibited high color contrast, quick switching kinetics, improved coloration efficiencies $\left(150 \mathrm{~cm}^{2} \mathrm{C}^{-1}\right.$ at $650 \mathrm{~nm}$ and $255 \mathrm{~cm}^{2} \mathrm{C}^{-1}$ at $1300 \mathrm{~nm}$ ), good long-time stability (reversibility of $98 \%$ after 500 cycles), and wide EC spectral coverage for both the Vis and IR regions. Huang et al. ${ }^{285}$ also obtained $\mathrm{WO}_{3}$ hexagonal nanorods, although they used tungstate powder, commercial tungsten oxide powder, $\mathrm{H}_{2} \mathrm{O}_{2}$, oxalic acid, $\mathrm{Na}_{2} \mathrm{SO}_{4}$ for the synthesis of nanorods and nanofibers via the hydrothermal method. Guo et al. ${ }^{286}$ obtained nanowires; however, they also used graphene sheets and ethanol as the solvent. Park et al. ${ }^{287}$ synthesized urchin-like $\mathrm{W}_{18} \mathrm{O}_{49}$ particles via the

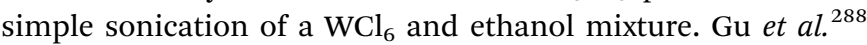
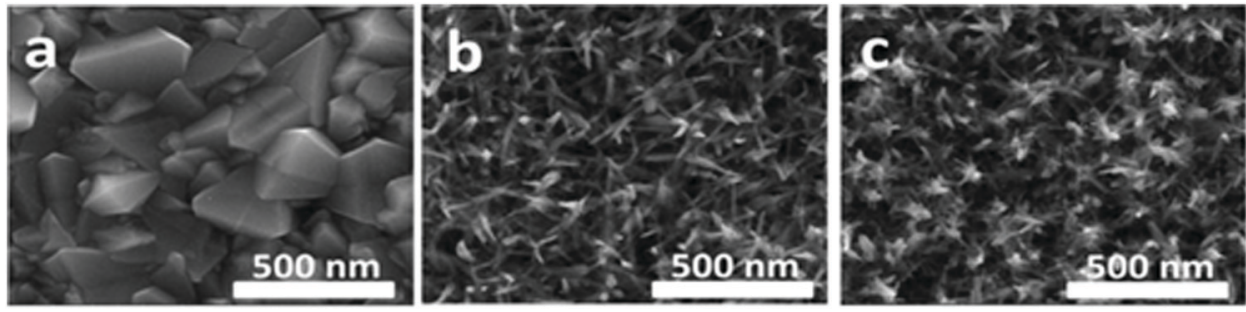

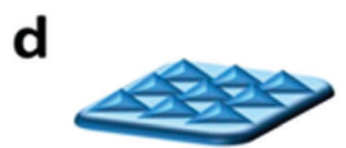

Bare FTO

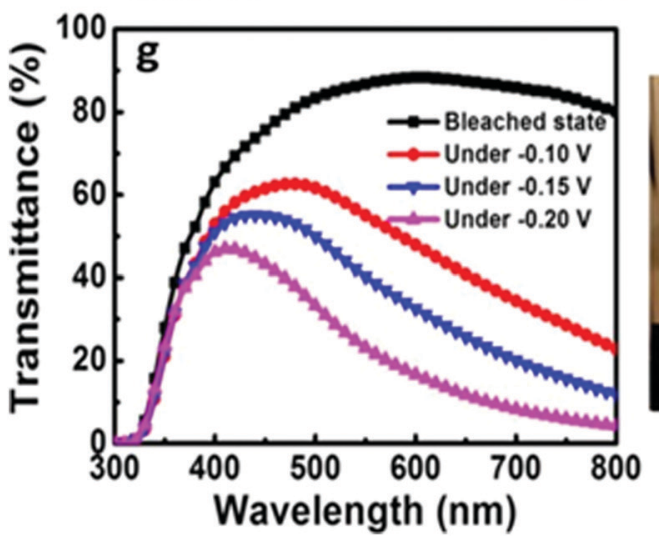

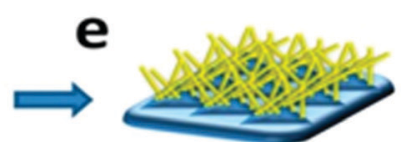

First step solvothermal h

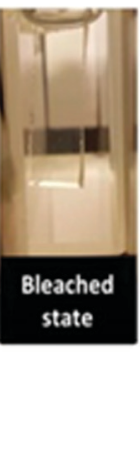

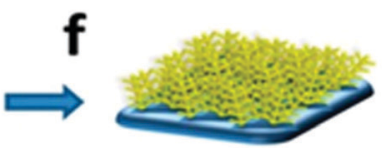

Second step solvothermal

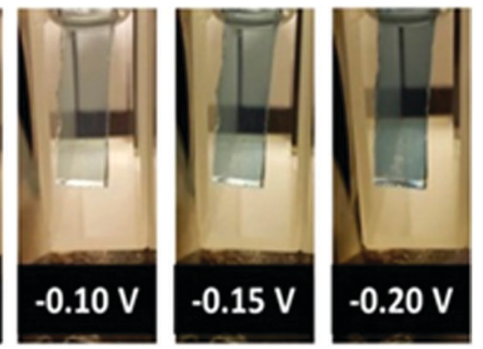

Fig. 9 (a-c) SEM images of $\mathrm{WO}_{3}$ nanowires and nanotree grown on FTO and (d-f) corresponding schematic illustrations, respectively. (g) UV-vis spectra of $\mathrm{WO}_{3}$ nanotree at various potential and photographs of its color change. Reproduced with permission. ${ }^{263}$ Copyright@2018, ACS. 
synthesized hierarchical structures composed of 1D nanowires/ nanorods via a simple hydrothermal method with various surfactants, e.g., $\mathrm{K}_{2} \mathrm{SO}_{4}, \mathrm{Rb}_{2} \mathrm{SO}_{4}, \mathrm{Na}_{2} \mathrm{SO}_{4}$ and $\left(\mathrm{NH}_{4}\right)_{2} \mathrm{SO}_{4}$. The urchin-like and ribbon-like structures were produced with $\mathrm{K}_{2} \mathrm{SO}_{4}$ and $\mathrm{Rb}_{2} \mathrm{SO}_{4}$ based on a sulfate-induced oriented growth mechanism. Guo et al. ${ }^{289}$ synthesized $\mathrm{W}_{18} \mathrm{O}_{49}$ nanostructures with different morphologies, i.e., nanowires, nanotubes, and nanoparticles, via the solvothermal method (Fig. 10a-i) by changing the alcohol and tungsten source $\left(\mathrm{WCl}_{6}\right.$ and $\left.\mathrm{W}\left(\mathrm{C}_{2} \mathrm{OH}\right)_{6}\right)$. They reported that using primary alcohol resulted in $\mathrm{W}_{18} \mathrm{O}_{49}$ nanostructures, whereas pentanol and secondary alcohols, e.g., 2-propanol and 2-butanol, gave a mixture of $\mathrm{WO}_{3}-\mathrm{W}_{18} \mathrm{O}_{49}$. The dispersed $\mathrm{W}_{18} \mathrm{O}_{49}$ particle suspension was paint coated on quartz glass using an applicator (Fig. 10j).
We also attempted to use similar routes to synthesize 1D stoichiometric and nonstoichiometric tungsten oxide with different morphologies (nanotubes (NT), nanowires (NW), nanowire bundles (NWB), nanorods (NR), nanoplates (NP), and urchin-like nanospheres (Fig. 11a-i). The different morphologies were tuned by changing a variety of pertinent variables such as organic/inorganic surfactants $\left(\mathrm{K}_{2} \mathrm{SO}_{4}, \mathrm{Na}_{2} \mathrm{SO}_{4}, \mathrm{Li}_{2} \mathrm{SO}_{4}\right.$, $\left(\mathrm{NH}_{4}\right)_{2} \mathrm{SO}_{4}, \mathrm{KCl}, \mathrm{KNO}_{3}$, citric acid, oxalic acid, PVP, and CTAB), temperature $\left(120-180{ }^{\circ} \mathrm{C}\right)$, reaction time (1-24 h), tungsten salt, $\left(\mathrm{Na}_{2} \mathrm{WO}_{4} \cdot 2 \mathrm{H}_{2} \mathrm{O}\right.$ and $\left.\mathrm{WCl}_{6}\right)$ and solvent (water, ethanol and ethylene glycol). Controlled experiments resulted in highly crystalline $1 \mathrm{D} \quad \mathrm{WO}_{3}$ nanostructures. The Brunauer-EmmettTeller (BET) surface area was derived for the $\mathrm{h}-\mathrm{WO}_{3}$ nanotubes $\left(71.95 \mathrm{~m}^{2} \mathrm{~g}^{-1}\right), \mathrm{WO}_{3-x}$ nanowires $\left(51.83 \mathrm{~m}^{2} \mathrm{~g}^{-1}\right), \mathrm{h}-\mathrm{WO}_{3}$
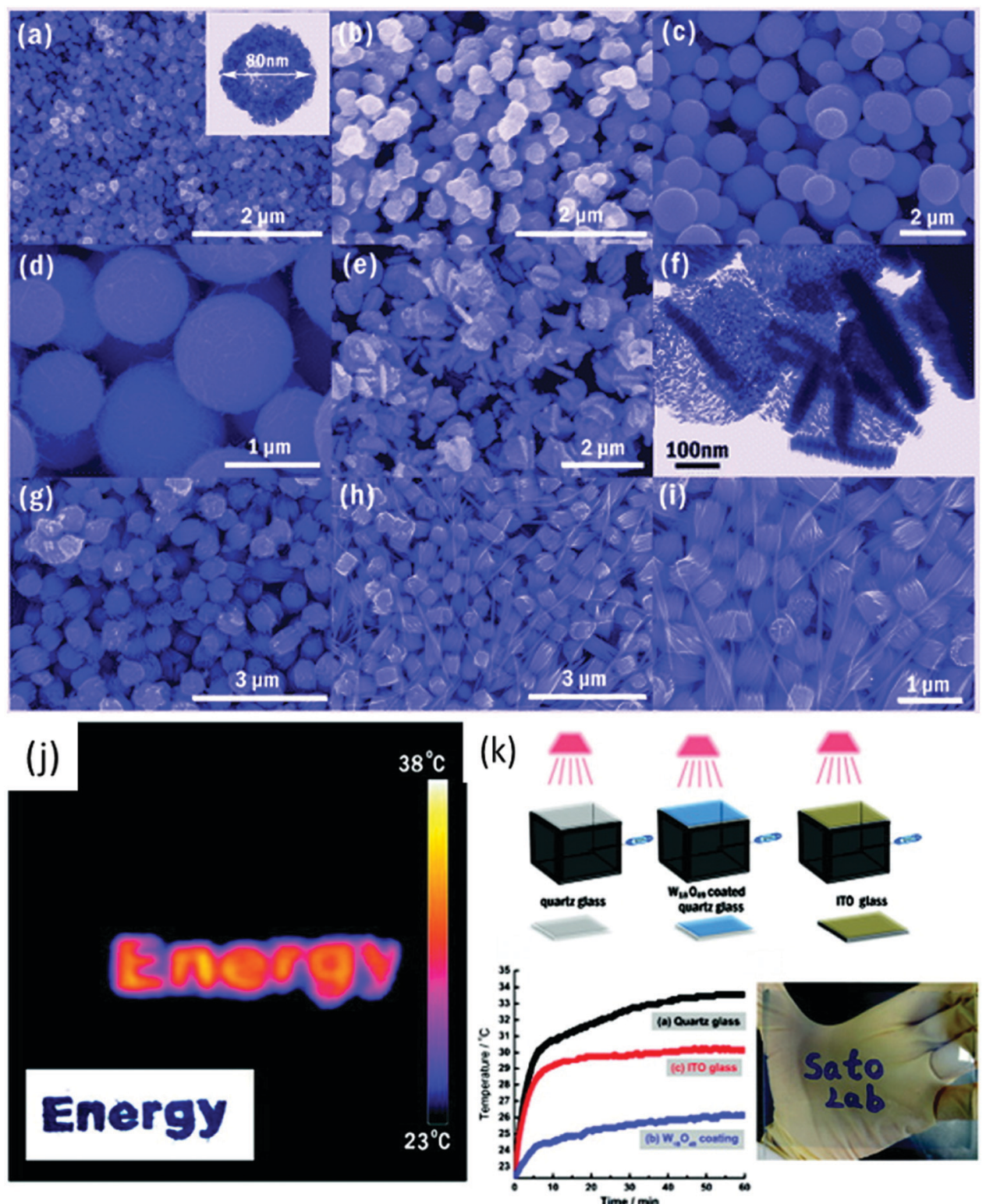

Fig. 10 (a-i) SEM and TEM images of the $\mathrm{W}_{18} \mathrm{O}_{49}$ nanomaterials. (j) Schematic illustration of the simulated experiment. Sealed boxes with a facet covered by quartz glass, $\mathrm{W}_{18} \mathrm{O}_{49}$ nanorods coated on quartz glass and ITO glass were irradiated by a $50 \mathrm{~W}$ halogen lamp. The temperature changes were determined using an electronic thermometer. (k) Time-dependent temperature in the box. Reproduced with permission. ${ }^{289}$ Copyright $\mathbb{C} 2012$, ACS. 

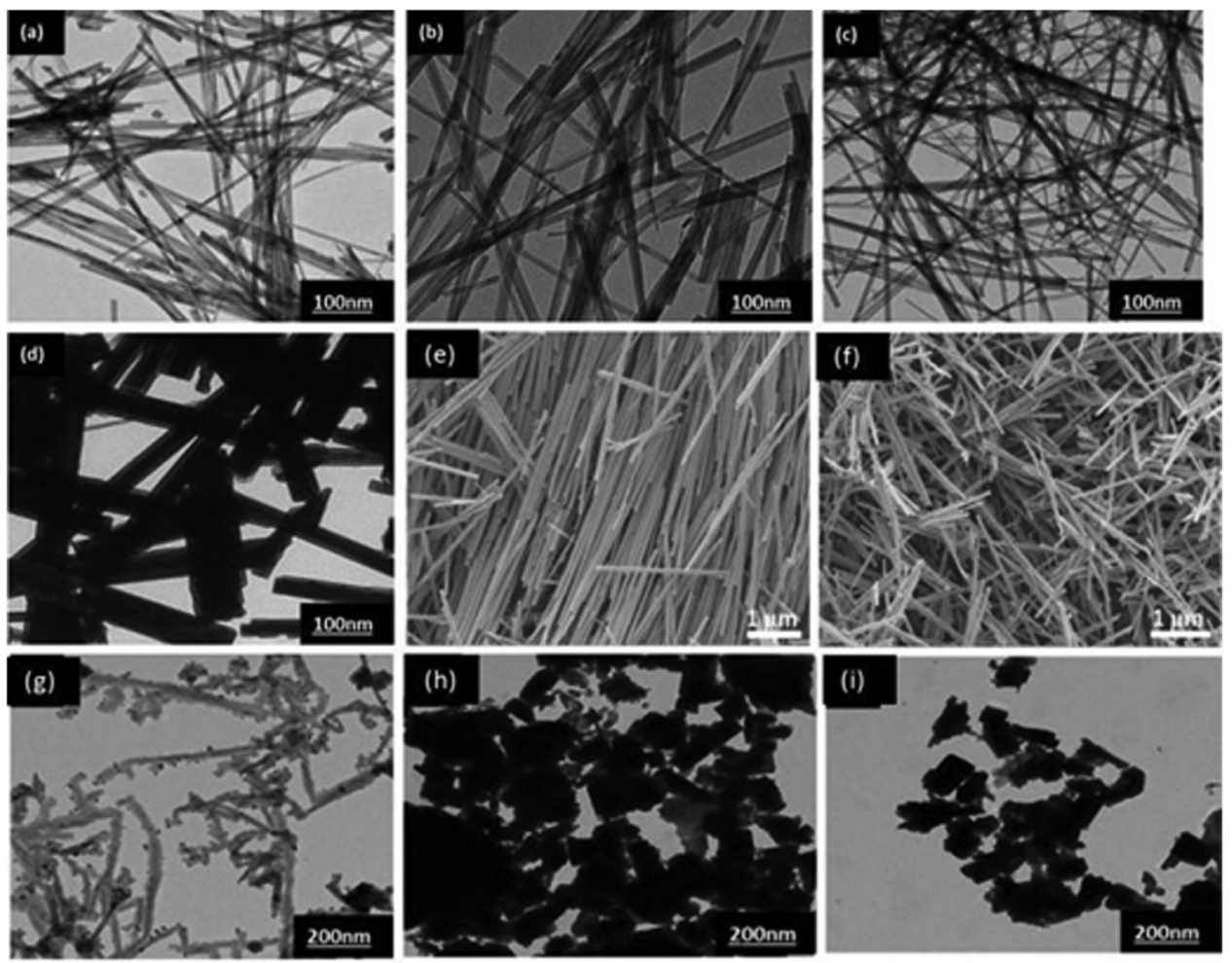

Fig. 11 TEM and SEM images of tungsten oxide nanoparticles: (a) $\mathrm{WO}_{3-x}-\mathrm{NW}$, (b) $\mathrm{WO}_{3}-\mathrm{NT}$, (c) $\mathrm{h}-\mathrm{WO}_{3}-\mathrm{NW}$, (d-f) $\mathrm{h}-\mathrm{WO}_{3}-\mathrm{NR}$, and (g) $\left(\mathrm{NH}_{4}\right)_{2} \mathrm{SO} \mathrm{O}_{4}$, and (h) and (i) $\mathrm{CA}$ and $\mathrm{OA}$ without $\mathrm{K}_{2} \mathrm{SO}_{4}$, respectively. Reproduced with permission. ${ }^{152}$ Copyright@2020, Elsevier.

nanowires $\left(42.70 \mathrm{~m}^{2} \mathrm{~g}^{-1}\right)$, nanorods $\left(24.60 \mathrm{~m}^{2} \mathrm{~g}^{-1}\right)$, and nanowire bundle ordered structures $\left(18.4 \mathrm{~m}^{2} \mathrm{~g}^{-1}\right)$. The effects of pertinent variables such as surfactants were explored by carrying out a series of experiments. Under hydrothermal conditions, the ions in the solutions and organic acids (citric acid and oxalic acid) adsorbed on the $\mathrm{WO}_{3}$ crystal facets and produced highly oriented $1 \mathrm{D} \mathrm{WO}_{3}$ nanostructures. Meanwhile, the $\mathrm{K}^{+}, \mathrm{Na}^{+}, \mathrm{Li}^{+}$and $\mathrm{SO}_{4}{ }^{2-}$ ions adsorbed on the $\mathrm{WO}_{3}$ crystal planes of $\mathrm{h}-\mathrm{WO}_{3}{ }^{143}$ The $\mathrm{SO}_{4}{ }^{2-}$ anions preferentially adsorbed on the surface parallel to the $c$ axis at the $\langle 001\rangle$ plane of $\mathrm{WO}_{3}$, resulting in the formation of $1 \mathrm{D}$ single-crystal nanostructures. ${ }^{195} \mathrm{CA}$ and OA allowed the controlled growth and conversion of the crystal phases. It was concluded that an appropriate number of ions and CA and OA played a crucial role in controlling the nanocrystal phases and crystallinity. Moreover, ultrathin aggregated nanotubes and nanowires were produced, which then simultaneously assembled into bundles due to the self-assembly and oriented attachment mechanism. ${ }^{288}$

Zheng and co-workers ${ }^{290}$ reported various morphologies of $\mathrm{WO}_{3}$, i.e., nanorods, nanofibers, and nanoflakes, by changing the surfactant and reaction conditions, as shown in Fig. 12a-d.
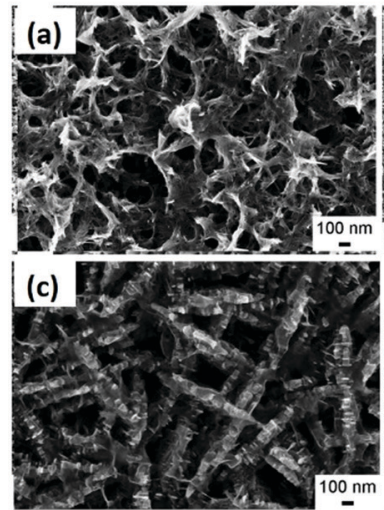
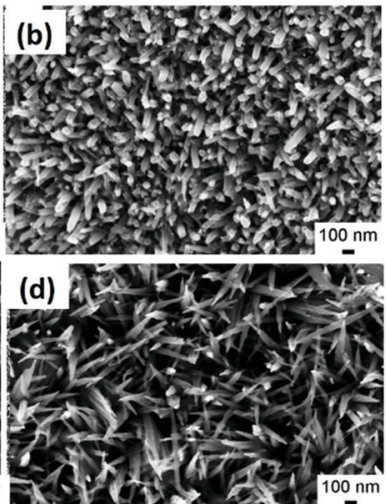

(e)

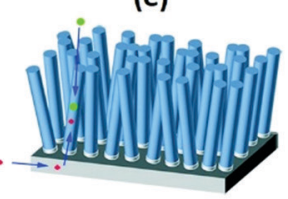

(f)

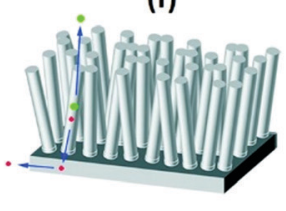

tungsten oxide octahedron

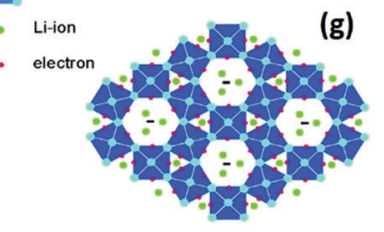

at the coloration stage

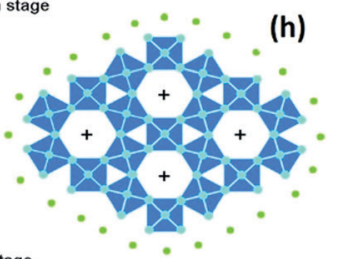

Fig. 12 SEM images of (a) porous $W_{3}$ nanofibers, (b) WNRs-1, (c) WO 3 nanoflake arrays, (d) WNRs-2. (e) EC mechanism of the WNRs the interface reaction process of $\mathrm{Li}$-ions and electrons between $\mathrm{WO}_{3}$ and the electrolyte and the diffusion process of $\mathrm{Li}$-ions in the $\mathrm{WO}_{3} \mathrm{Crystal}$ lattice. Reproduced with permission. ${ }^{290}$ Copyright@2015, RSC. 
The impact of crystallinity and morphologies on the EC properties was also investigated. Accordingly, nanorods were grown via seed-mediated growth on an FTO substrate at $\mathrm{pH} 2$ via the hydrothermal process without any hard organic templates, followed by annealing (Fig. 12b). The nanofibers grew on FTO at $\mathrm{pH} 2.4$ with the assistance of $\mathrm{KCl}$ as a capping agent, keeping the temperature $160{ }^{\circ} \mathrm{C}$ for $4 \mathrm{~h}$ (Fig. 12c). For the nanoflakes, the $\mathrm{pH}$ was 1.5 and $\mathrm{NaCl}$ is used as the capping agent at $180{ }^{\circ} \mathrm{C}$ for $40 \mathrm{~min}$ (Fig. 12d). The $\mathrm{WO}_{3}$ nanorods grown on FTO showed a high optical modulation of $64 \%$, coloration efficiency of $61 \mathrm{~cm}^{2} \mathrm{C}^{-1}$, and switch speeds of $5 \mathrm{~s}$ and $6 \mathrm{~s}$ as shown in Fig. 12e-h. It was reported that the highly ordered $\mathrm{WO}_{3}$ nanorods grown on an FTO seed layer showed the best EC performance among the morphologies. High-temperature annealing also affected the EC properties, given that the annealed $\mathrm{WO}_{3}$ nanorod sample possessed a low optical modulation due to its long hexagonal tunnel, but short response time due to its high crystallinity.

\section{Strategies to improve EC properties}

\section{1. $\mathbf{W O}_{2.72}$ hybridized/mixed composites}

It is believed that composite materials can aggregate and boost the performance of two/more ingredients. The mixing of two active materials with matching behavior can achieve a dominant interfacial area to a large extent. In nanoscale composites, the structural organization is advantageous because the physicochemical features arise from the confined interfaces. However, in arbitrary mixing, even the active components of material blends may be affected/detrimental due to the fact that their passageways are tortuous, and there may be some inactive sites. These composites may be more beneficial for the deliberate design of architectures from their mesoscale structure. $^{291,292}$ The metal oxide nanocomposites with template mesoscale architectures have fast switching kinetics and long cycle life. In this case, it has been reported that two degenerate metal oxide nanocrystals may efficiently modulate the NIR spectral transmittance via electrochemical charging, and then discharging of free electrons, which are responsible for LSPR trigger absorption. ${ }^{40,293,294}$ Hence, it can be concluded that NIR spectral light is much more intense at shorter wavelengths (700-1300 nm), which can be ideal for composite nanocrystals with LSPR absorption, and thus beneficial for dynamic solar light.

Polyoxometalate nanoclusters (POMs) denote a familiar class of metal oxide cluster compounds with framework topologies, which possess electronic and optical properties. ${ }^{295,296}$ POMs as an electron reservoir undergo reversible multielectron transfer reactions and significantly impart EC properties. Gu et al. ${ }^{122}$ studied the EC performance of crown-type polyoxometalates, $\mathrm{K}_{28} \mathrm{Li}_{5} \mathrm{H}_{7} \mathrm{P}_{8} \mathrm{~W}_{48} \mathrm{O}_{184} \cdot 92 \mathrm{H}_{2} \mathrm{O} \quad\left(\mathrm{P}_{8} \mathrm{~W}_{48}\right)$, and $\mathrm{W}_{18} \mathrm{O}_{49}$ nanowires coated on ITO glass via the LbL process, as shown in Fig. 13. The $\mathrm{P}_{8} \mathrm{~W}_{48}$ anion is a visible light absorption material owing to its wide range of stability. In the reduced state, it may exhibit good absorptivity of visible light, while the $\mathrm{W}_{18} \mathrm{O}_{49}$ NWs are a candidate with excellent absorptivity of NIR light. The effects of the $\mathrm{W}_{18} \mathrm{O}_{49}$ NWs and $\mathrm{P}_{8} \mathrm{~W}_{48}$ on nearinfrared and visible-light selective modulation were studied via the self-assembly technique. Meanwhile, the $\mathrm{W}_{18} \mathrm{O}_{49} \mathrm{NWs}$ or $\mathrm{P}_{8} \mathrm{~W}_{48}$ anion are negatively charged species, which hybridized with the positively charged poly(ethylenimine) (PEI)
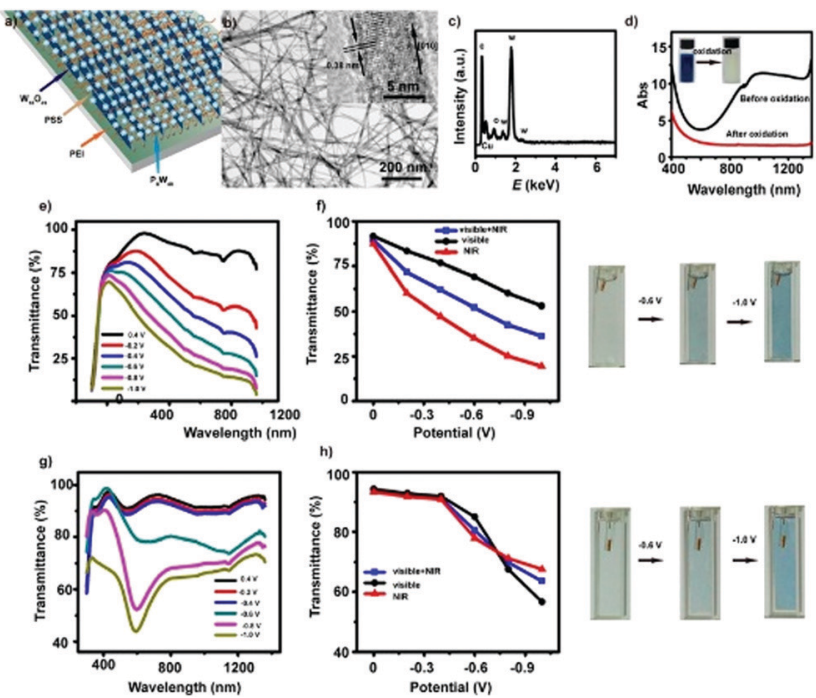

(i)

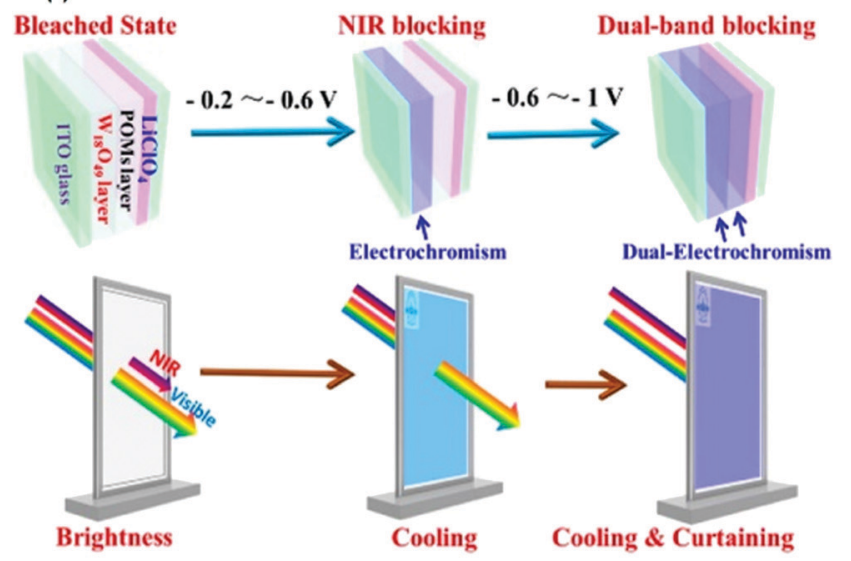

Fig. 13 Transmittance spectra of $\left[\mathrm{PSS}(\mathrm{PEI} / \mathrm{PSS})_{3}\left(\mathrm{PEI} / \mathrm{W}_{18} \mathrm{O}_{49}\right)_{30} /\left(\mathrm{PEI} / \mathrm{P}_{8} \mathrm{~W}_{48}\right)_{20}\right]$ film at different potentials, held for 4 min. (b) Relative transmittance change for $\left[\mathrm{PSS}(\mathrm{PEI} / \mathrm{PSS})_{3}\left(\mathrm{PEI} / \mathrm{W}_{18} \mathrm{O}_{49}\right)_{30} /\left(\mathrm{PEI} / \mathrm{P}_{8} \mathrm{~W}_{48}\right)_{20}\right]$ film under different applied potentials; blue curve: NIR + Vis region, red curve: NIR region, and black curve: visible region, and digital photographs of the film at $0.4 \mathrm{~V},-0.6 \mathrm{~V}$, and $-1.0 \mathrm{~V}$, respectively. (c and d) In situ optical response of [PSS(PEI/ $\left.\mathrm{PSS})_{3}\left(\mathrm{PEI} / \mathrm{W}_{18} \mathrm{O}_{49}\right)_{30} /\left(\mathrm{PEI} / \mathrm{P}_{8} \mathrm{~W}_{48}\right)_{20}\right]$ multilayer when switching between $-0.60 \mathrm{~V}(100 \mathrm{~s})$ and $-1.0 \mathrm{~V}(100 \mathrm{~s})$ to $0.4 \mathrm{~V}(200 \mathrm{~s})$, where each step is measured at $1060 \mathrm{~nm}$ (c) and $500 \mathrm{~nm}$ (d). (e and f) Optical density variation as a function of charge density at $1060 \mathrm{~nm}$ (red curve) and $500 \mathrm{~nm}$ (black curve). The applied potential is $-0.6 \mathrm{~V}$ (e) and $-1.0 \mathrm{~V}$ (f) for $100 \mathrm{~s}$. Corresponding schematic (i) shows the dual-band optical modulation. Reproduced with permission. ${ }^{122}$ Copyright@2018, ACS. 
polymer to create electrostatic interactions for alternating layer deposition of a multilayered film (abbreviated as $\left(\mathrm{PEI} / \mathrm{W}_{18} \mathrm{O}_{49}\right)_{n}$ or $\left.\left(\mathrm{PEI} / \mathrm{P}_{8} \mathrm{~W}_{48}\right)_{n}\right)$. The ITO glass was first activated via the LbL deposition of a polyelectrolyte multilayer of poly(sodium 4-styrene-sulfonate) (PSS) and PEI to increase the surface charges and surface affinity before the deposition of the (PEI/ $\left.\mathrm{W}_{18} \mathrm{O}_{49}\right)_{n}$ or $\left(\mathrm{PEI} / \mathrm{P}_{8} \mathrm{~W}_{48}\right)_{n}$ composite multilayer thin film. Due to the excellent stability of the films prepared using $\mathrm{P}_{8} \mathrm{~W}_{48}$ and $\mathrm{W}_{18} \mathrm{O}_{49}$ and their precise structural control, the nanocomposites film achieved the high coloration efficiency of $42.31 \mathrm{~cm}^{2} \mathrm{C}^{-1}$ at $500 \mathrm{~nm}$ and $287.49 \mathrm{~cm}^{2} \mathrm{C}^{-1}$ at $1060 \mathrm{~nm}$ (Fig. 13e-h). The switching speeds for the transparent, NIR-blocking and broadband-blocking were $86 \mathrm{~s}, 52 \mathrm{~s}$, and $26 \mathrm{~s}$, respectively. The electrochemical cycle stability of the composite film after 500 cycles at wavelengths of $1060 \mathrm{~nm}$ and $500 \mathrm{~nm}$ was only $3.20 \%$ and $2.40 \%$, respectively. The obtained results provide an effective way for the formation of compositebased functional smart glass.

Liu et $a .^{257}$ and Wang et al. ${ }^{119}$ demonstrated that ITO-based coating films are hard, which can be damaged and lose conductivity after a certain degree of bending of the device structures. They used $\mathrm{Ag}$ nanowires as a conductive substrate support and deposited $\mathrm{W}_{18} \mathrm{O}_{49}$ nanowires for EC device applications. The $\mathrm{Ag}$ nanowires and $\mathrm{W}_{18} \mathrm{O}_{49}$ nanowires were orderly assembled via the Langmuir-Blodgett (LB) $\operatorname{method}^{257}$ to develop several layers with elevated conductivity and transmittance. The $\mathrm{Ag}-\mathrm{W}_{18} \mathrm{O}_{49}$ nanowire co-assembled thin film obtained via the LB method was continuously transferred to a PET substrate as an EC smart cell electrode, as demonstrated in Fig. 14. An increase in the number of layers of $\mathrm{W}_{18} \mathrm{O}_{49}$ nanowires significantly increased the coloration efficiency, cyclic stability, and transmissivity, as shown in Fig. 14b, c, f and g. The $\mathrm{Ag}-\mathrm{W}_{18} \mathrm{O}_{49}$ co-assembled nanowire film could bend to a very small radius of $1.2 \mathrm{~cm}$ without any mechanical loss, which showed an excellent EC performance and mechanical stability (1000 cycles), as shown in Fig. 14d and e.
An $\mathrm{Ag}-\mathrm{W}_{18} \mathrm{O}_{49}$ co-assembled film was used to develop a multilayered nanowire network on an ITO-PET film over a large-area conductive substrate with tunable conductivity (7-40 $\Omega \mathrm{sq}^{-1}$ ) and transmissivity (58-86\% at $\left.550 \mathrm{~nm}\right)$, as shown in Fig. 15a-e. ${ }^{119}$ The $\mathrm{Ag}-\mathrm{W}_{18} \mathrm{O}_{49}-\mathrm{LiClO}_{4} / \mathrm{PMMA} / \mathrm{PC}-\mathrm{ITO}-\mathrm{PET}$ hybrid all-in-one solid-state portable and flexible EC device is shown in the colored and bleached states, which could be bent to a radius of about $1.2 \mathrm{~cm}$ with $90 \%$ resistance retention $(\Delta R / R \approx 8.3 \%)$ after more than 1000 cycles. Heo et al. ${ }^{123}$ produced a low-temperature mesoporous $\mathrm{WO}_{2.72}-\mathrm{NbO}_{x}$ composite thin film on an ITO-PET supporting substrate, as shown in Fig. 16. Consequently, they realized a random/disordered arrangement of nanocrystal structures in organic media, which giving the opportunity to develop mesoporosity and overcoming the compact structure formulations (Fig. 16E and F). Ligand-stabilized $\mathrm{WO}_{2.72}$ nanorods were synthesized using $\mathrm{W}(\mathrm{CO})_{6}$ and $\mathrm{Me}_{3} \mathrm{NO}$ dissolved in oleylamine in a three-neck flask in an $\mathrm{N}_{2}$ glove box and annealing directly at $300{ }^{\circ} \mathrm{C}$ for $90 \mathrm{~min}$. After the wetchemical reaction, the colloidal solution was washed using isopropyl alcohol (IPA) and redispersed in toluene, which were repeated several times to produce clean high aspect ratio $\mathrm{WO}_{2.72}$ nanorods. They also synthesized low aspect ratio $\mathrm{WO}_{2.72}$ nanorods by reducing the $\mathrm{Me}_{3} \mathrm{NO}$ and oleylamine concentration, as shown in Fig. 16A. The obtained $\mathrm{WO}_{2.72}$ may contain highly encapsulated ligands, which were stripped using Meerwein's salts $\left(\mathrm{Et}_{3} \mathrm{OBF}_{4}\right) . \mathrm{Et}_{3} \mathrm{OBF}_{4}$ was dissolved in acetonitrile ( $\mathrm{AN}$ ) and mixed with $\mathrm{WO}_{2.72}$ nanorods in toluene at a $1: 1$ ratio with constant stirring, centrifuged and then redispersed for repeated washing. Both the ligand-stripped and ligand-capped $\mathrm{WO}_{2.72}$ nanorods were spin-coated on clean ITO-PET substrates using different dispersion media to form thin films of about $300 \mathrm{~nm}$. The results showed that ligandstripped $\mathrm{WO}_{2.72}$ nanorods produced a thin mesoporous film due to the opposite electrostatic links, which prevented the spontaneous packing of the nanorods (Fig. 16E and F). After the fabrication of the $\mathrm{WO}_{2.72}$ nanorod thin film, a guest component
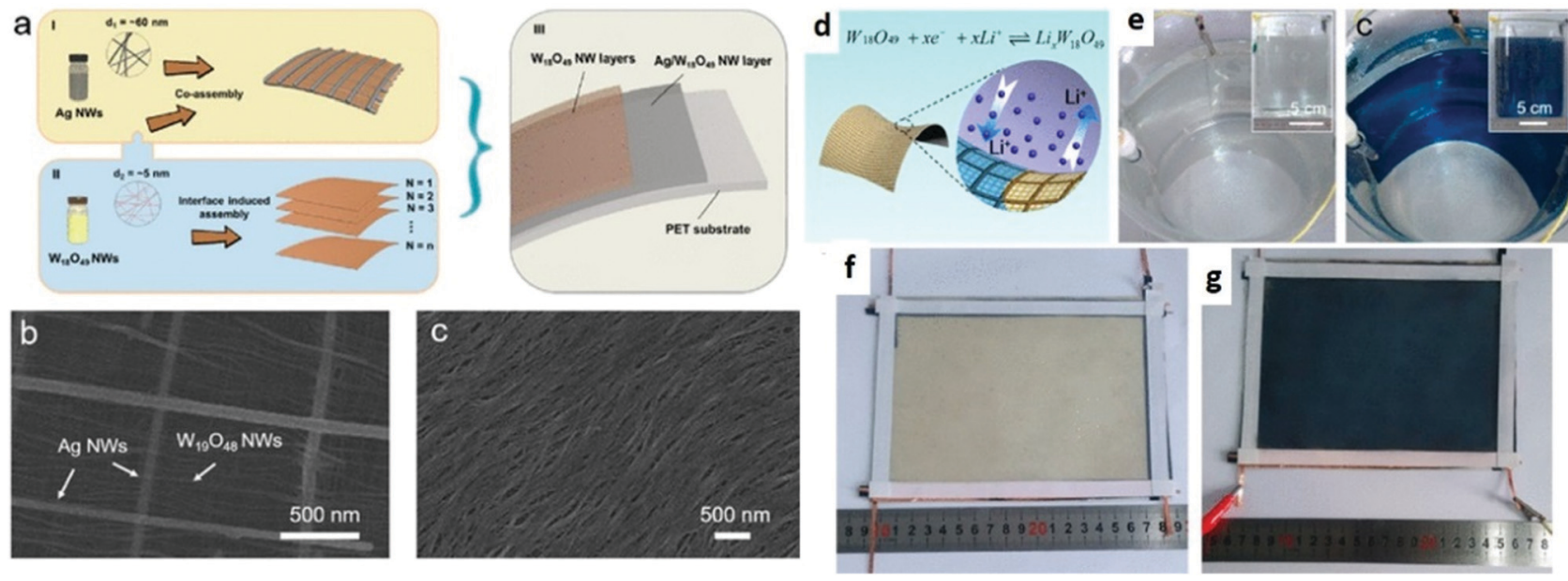

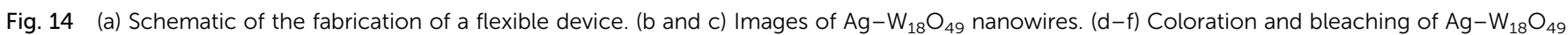
nanowires film before and after an applied potential. Reproduced with permission. ${ }^{119}$ Copyright@2017, ACS. 

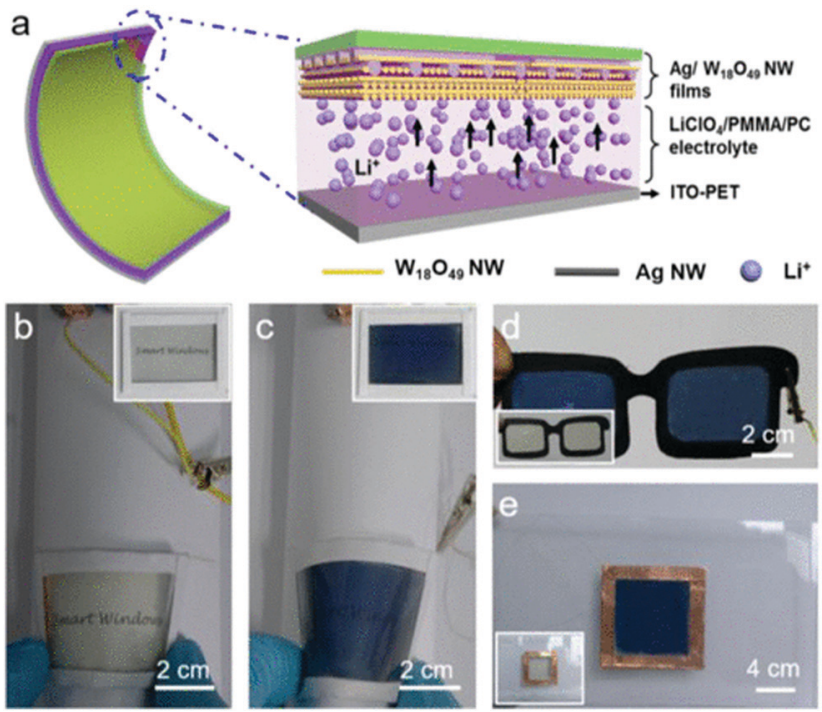

Fig. 15 (a) Schematic diagram of a solid EC device. Photographs showing the (b) bleached and (c) colored state of the EC devices in the bending state. The insets are photos without bending. (d) Photographs of the EC glasses model and (e) EC window model. Reproduced with permission. ${ }^{119}$ Copyright $(2017$, ACS.

solution composed of $\mathrm{NbO}_{2} \mathrm{POM}$ clusters and $\left[\mathrm{N}\left(\mathrm{CH}_{3}\right)_{4}\right]_{6}$ $\mathrm{Nb}_{10} \mathrm{O}_{28} \cdot 6 \mathrm{H}_{2} \mathrm{O}$ was drop-casted on the fabricated film, and then immersed in a formic acid and absolute ethanol mixed solution and heated at $150{ }^{\circ} \mathrm{C}$. The $\mathrm{WO}_{x}-\mathrm{NbO}_{x}$ composite film fabricated on ITO-PET has the advantages of selective control of the VisNIR light transmission, fast switching, and long cycle life (Fig. 16G and H).

Kim et al. ${ }^{41}$ designed $\mathrm{WO}_{3-x}$ nanocrystals and an amorphous niobium oxide nanocomposite mesoporous film. The obtained $\mathrm{WO}_{3-x}-\mathrm{NbO}_{x}$ composite exhibited high charge-discharge kinetics and stability for about 2000 cycles. However, wet-synthesis of $\mathrm{WO}_{3-x}$ nanocrystals via the direct heating of a mixture of tungsten salts without applied pressure requires harsh conditions, high temperature, and the product yield is small. Vuong et al. ${ }^{297}$ also employed similar procedures and reported the fabrication of a porous core-shell $\mathrm{TiO}_{2}-\mathrm{WO}_{3}$ nanowire structure film via sputter deposition using carbon nanotubes as a substrate and frequent thermal treatment. The particle size, morphology, electrochemical, and EC performance were studied and then compared with that of thin $\mathrm{TiO}_{2}-\mathrm{WO}_{3}$ composite films and single $\mathrm{WO}_{3}$ and $\mathrm{TiO}_{2}$ nanowires. The porous composite nanowires exhibited high proton intercalation capability with good reversible electrochemical cycles through intercalation and de-intercalation of the chargecompensating ions. Dulgerbaki and co-workers ${ }^{298}$ fabricated $\mathrm{WO}_{3} /$ PEDOT hybrid films and $\mathrm{Li}$ co-workers ${ }^{299}$ fabricated $\mathrm{W}_{0.71} \mathrm{Mo}_{0.29} \mathrm{O}_{3} /$ PEDOT:PSS hybrid film-modified electrodes, which exhibited highly improved EC performances, including fast switching kinetics, high coloration contrast, and tremendous coloration efficiency of $>100 \mathrm{~cm}^{2} \mathrm{C}^{-1}$ compared with the pure $\mathrm{WO}_{3}$ films.

\section{2. $\mathrm{WO}_{3-x} / \mathrm{metal} / \mathrm{WO}_{3} /$ other EC bilayer architecture films}

To strengthen the transparency and EC functionalities, soft coatings as dielectric/metal/dielectric (DMD) blends have been appealing in recent years as alternatives to ITO-coated substrates. ${ }^{300}$ Various dielectric materials, for example $\mathrm{WO}_{3}$, $\mathrm{TiO}_{2}, \mathrm{MoO}_{3}$, and $\mathrm{ZnS}$, have been reported for the construction of DMD blends recently. The DMD assembly has also been extensively used in electrodes in organic optoelectronic devices, such as light-emitting diodes and organic solar cells. ${ }^{301-303}$ In DMD coatings, the $\mathrm{WO}_{3}$ film may perform a dual function, where it is anti-reflective for light in the transparent state and becomes absorbent in the colored state. ${ }^{293,304-307}$ Furthermore, DMD bilayer or trilayer films possess optimum resistivity $(>15$ $\Omega)$, high transparency $(<80 \%)$ in the visible region, low thickness and superior flexibility. ${ }^{124,125,305-307}$ For dual functionality, the matrix design by simulation of $\mathrm{D} / \mathrm{M} / \mathrm{WO}_{3}$ as glass/ZnS
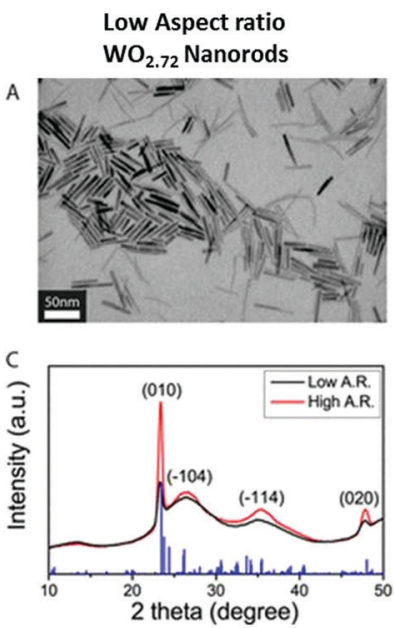
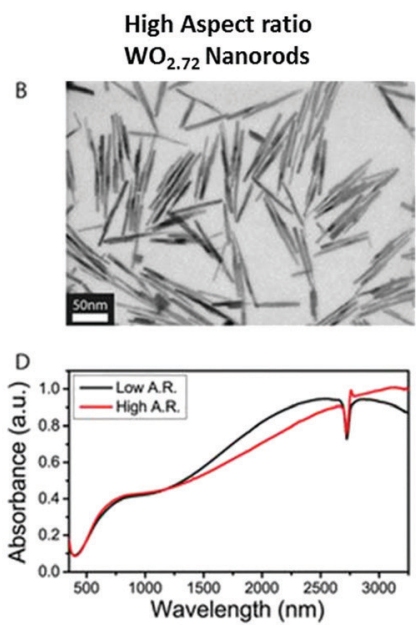

Template-Free Mesoporous $\mathrm{WO}_{2.72}$ Film
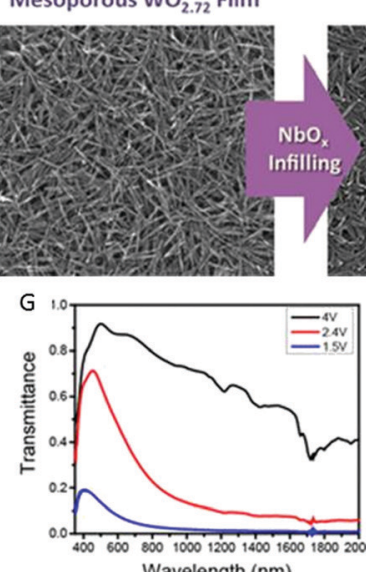

Wavelength $(\mathrm{nm})$
Flexible Dual Band Electrochromic Film
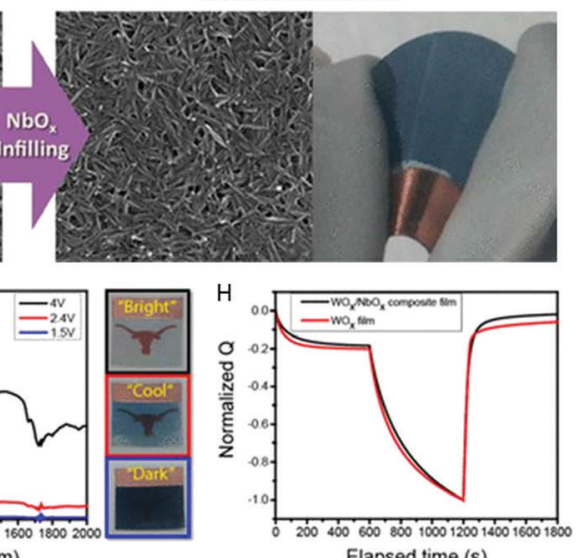

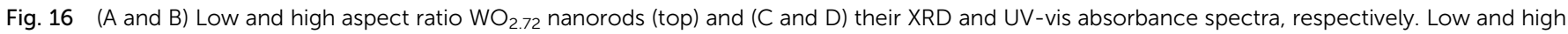

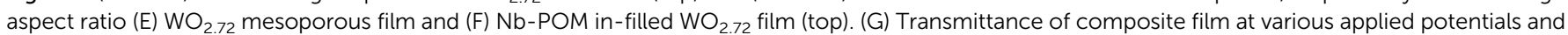
their corresponding photographs. $(\mathrm{H})$ Charging and discharging profile at various potentials. Reproduced with permission. ${ }^{123}$ Copyright $C 2017$, ACS. 
(34 nm)/Ag (15 nm)/WO $\mathrm{WO}_{3}$ (326 nm) was formulated and verified $\mathrm{v}$ experiments by Leftheriotis' team. ${ }^{304}$ The color coating simulation studies showed the asymmetry of the radiation propagation path, which is absorptive in one direction and highly reflective in the opposite direction. They also substituted the layer of $\mathrm{ZnS}$ with an optimum thickness of $\mathrm{WO}_{3}$ and the $\mathrm{Ag}$ layer with $\mathrm{Au}$, and the addition of a 4 th protective layer of $\mathrm{Al}_{2} \mathrm{O}_{3}$ demonstrated feasible replacements toward the optimum design. The film was produced via electron-beam gun deposition with a transparency of $57 \%$ and sheet conductivity of $9 \Omega$. The obtained results showed that the outer $\mathrm{WO}_{3}$ coating layer should be thick enough to protect the $\mathrm{Ag}$ nanolayer from the liquid electrolyte. The optical contrast was $90 \mathrm{~cm}^{2} \mathrm{C}^{-1}$ and a coloration efficiency of $30 \mathrm{~cm}^{2} \mathrm{C}^{-1}$ was recorded for 500 coloration/bleach cycles. The influence of liquid electrolyte on the EC coatings was observed with an increase in resistance and stability due to degradation, where the Ag nanolayer degraded because of the interactions of the electrolyte through the pores of the coated $\mathrm{ZnS}$ layers. ${ }^{308}$ Hence, a layer thickness of $\mathrm{WO}_{3}$ of up to $300 \mathrm{~nm}$ was adequate for the protection of the $\mathrm{Ag}$ nanolayer over the $\mathrm{ZnS} / \mathrm{Ag} / \mathrm{ZnS}$ stack.

Koubli et al. ${ }^{306}$ employed electron beam deposition for the formation of a $\mathrm{WO}_{3} / \mathrm{Ag} / \mathrm{WO}_{3}$ coating film. The fabricated film exhibited excellent deep coloration. However, with the intercalation of electrical charges, the film was unstable and damaged with the reversal of electrical potential. It became dysfunctional in the bleach state due to the reaction of $\mathrm{Ag}$ with $\mathrm{Li}^{+}$ions, and the resistance of the $\mathrm{Ag}$ layer increased. Hence, for safe mode operation, the charge density was lowered to $12.6 \mathrm{mC} \mathrm{cm}^{-2}$ from $15.7 \mathrm{mC} \mathrm{cm}^{-2}$. Li et al. ${ }^{305}$ demonstrated that a $\mathrm{WO}_{3} / \mathrm{Ag} / \mathrm{WO}_{3}$ film could sustain up to 3000 cycles without EC performance degradation, as shown in Fig. 17. The poor adhesion and rapid degradation regarding color/bleaching of the bifunctional $\mathrm{WO}_{3} / \mathrm{Ag} / \mathrm{WO}_{3}$ film were improved by sputtering deposition using metal targets. ${ }^{125}$ They successfully added an ultrathin tungsten sacrificial layer followed by an external $\mathrm{WO}_{3}$ layer. Metal-to-metal bonds are generally stronger than metalto-oxygen bonds. Therefore, the Ag-to-W layer film was adjusted to the desired thickness and the resulting film in the 2000th bleaching cycle showed a stable EC performance and avoided oxidation of the silver metal nanolayer in the oxygen atmosphere. Based on this strategy, the oxidation of silver metal $\left(\mathrm{Ag}^{0}\right)$ can be eliminated and the conductivity of the film can be lost. Moreover, according to the conventional DMD three-layer structure, the introduction of a sacrificial $\mathrm{W}$ layer presents complexity to the deposition process in the form of a fourth structural layer in the device configuration.

Najafi-Ashtiani et al. ${ }^{309}$ used high-power impulse magnetron sputtering (HiPIMS) instead of DC/RF magnetron sputtering technology to produce aa DMD film without a fourth sacrificial metal layer, as illustrated in Fig. 18. The HiPIMS EC deposition method has a wide range of use in industry and production. Converting a traditional sputtering system to HiPIMS operation only requires the application of a suitable power supply to derive the target in the HiPIMS mode. ${ }^{310}$ The high instantaneous power supply produces high-density plasma of the sputtered target material with a high ionization degree. ${ }^{310}$ The limited supply of oxygen is promising for the controllable growth of $\mathrm{WO}_{3}$ nanoparticles and external layer deposition to reduce the oxidation of the Ag layer. ${ }^{309}$ The thickness of $\mathrm{WO}_{3}$ $(30 \mathrm{~nm}) / \mathrm{Ag}(10 \mathrm{~nm}) / \mathrm{WO}_{3}(50 \mathrm{~nm})$ was optimized with the sheet resistance of $\sim 23 \Omega$ and transmittance of $80 \%$ (Fig. 18a). The fabricated coating exhibited an EC performance with good transparency, excellent cycling stability for 2500 cycles, fast switching kinetics, and Vis-NIR light modulation (Fig. 18b).

The EC performance of DMD films is not satisfactory in terms of switching time, switching speed, optical modulation, and durability. To this end, the pioneering work of
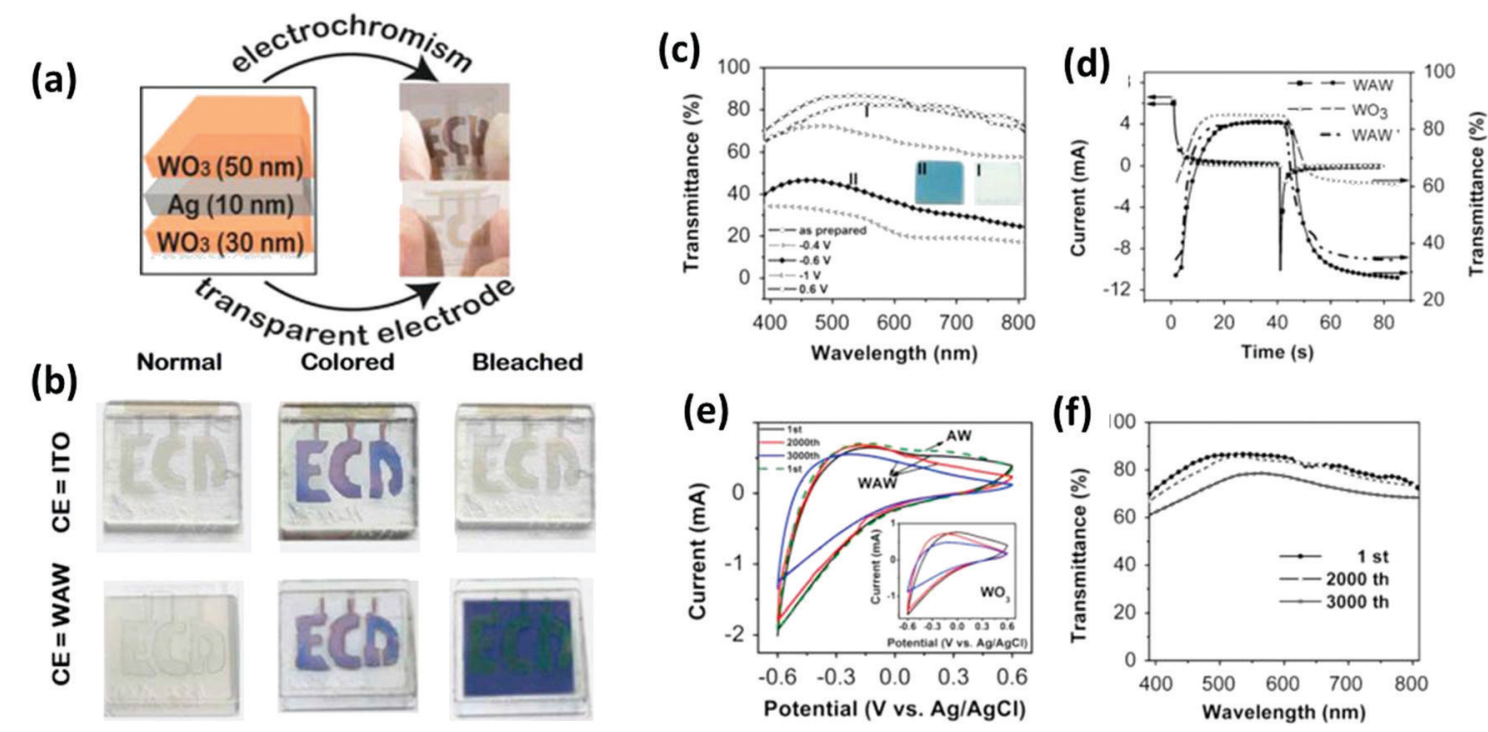

Fig. 17 (a and b) Different layer thickness of WAW (30/10/50 nm) used as an electrode and their corresponding color and bleached states. (c) Transmittance variations with a change in voltage ( 0.6 to $-0.6 \mathrm{~V})$. (d) Current and optical contrast with step potential. (e) CV curves and (f) transmittance variations with different cycles. Reproduced with permission. ${ }^{305}$ Copyright@2015, Elsevier. 

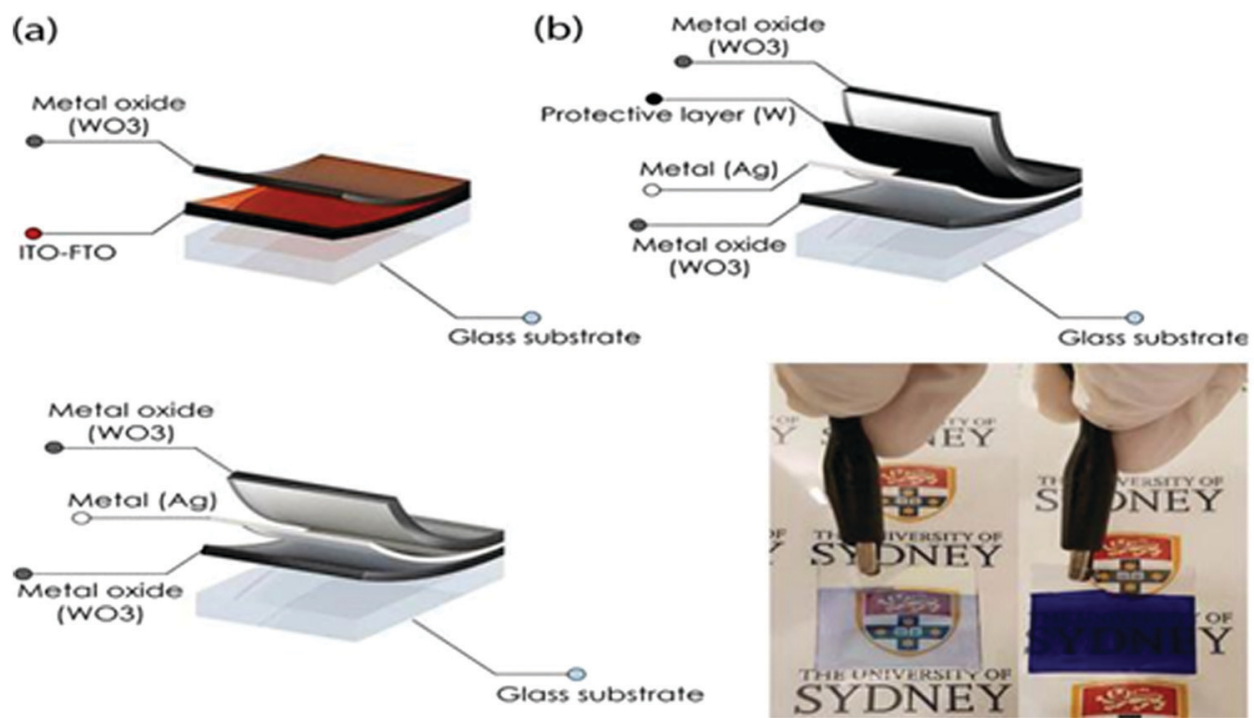

Fig. $18 \mathrm{HiPIMS}$-sputtered $\mathrm{EC} \mathrm{WO} / \mathrm{Ag} / \mathrm{WO}_{3}$ (DMD) three-layer structure. (a) $\mathrm{WO}_{3}$ deposited on ITO-FTO-coated glass. (b) Transparent, conductive DMD structure deposited on glass without ITO/FTO with a tungsten (W)-protective layer on the Ag layer. (c) DMD structure (ITO-FTO free) fabricated via HiPIMS without a protective layer. Optical images of bleached and colored states of the DMD (30 nm/10 nm/50 nm) coating. Reproduced with permission. ${ }^{309}$ Copyright@2019, ACS.

Dong et al. ${ }^{124}$ proved the application of mixed oxides of $\mathrm{W}-\mathrm{Mo}$ encapsulated $\mathrm{Ag}$ such as an $\mathrm{MoO}_{3}-\mathrm{WO}_{3} / \mathrm{Ag} / \mathrm{MoO}_{3}-\mathrm{WO}_{3}$ trilayer film for ITO-free EC devices, as demonstrated in Fig. 19. The film thickness was optimized at $40 \mathrm{~nm}$, resulting in the sheet resistance of $9 \Omega$ with a fast-switching speed and high coloration efficiency $\left(70 \mathrm{~cm}^{2} \mathrm{C}^{-1}\right)$.

\subsection{Doped $\mathrm{WO}_{2.72}$ nanostructures}

Using dopants or intended impurities to switch the behavior of nanomaterials is at the heart of various key technologies. For semiconductors, the doping process is crucial, otherwise they would be electrically insulating. Certain applications such as wavelength-tunable lasers, ${ }^{311}$ bioimaging, ${ }^{312}$ photocatalysis, and solar cells ${ }^{313}$ may eventually rely on the doping of nanocrystals to tune their electrical properties. Doping is an effective method to tailor the bandgap of semiconductor materials by introducing a transition state (defect band) between their valence bands and the conducting bands. Moreover, dopants can strongly alter the electronic, optical, and magnetic properties of bulk semiconductors. ${ }^{314}$ The EC efficiency of $\mathrm{WO}_{3-x}$ / $\mathrm{WO}_{3}$ has been tested by doping ${ }^{315}$ with $\mathrm{Mo}, \mathrm{Nb}, \mathrm{Ti}, \mathrm{Ni}, \mathrm{Al}, \mathrm{Ag}$, $\mathrm{Pd}, \mathrm{Cu}, \mathrm{Au}$, etc. The use of impurity atoms to tune the conductivity of $\mathrm{WO}_{3-x}$ can be better understood by considering (i) substitutional doping and (ii) interstitial doping.

5.3.1. Substitutional doping. Substitutional doping plays a key role in tuning the electrical conductivity in semiconductor materials, where the dopant atom replaces an atom in a nanocrystal, thereby integrating discrete components into circuits and devices. ${ }^{316}$ Semiconducting nanocrystals possess low free carrier concentrations, and thus do not exhibit LSPR phenomena. Accordingly, substitutional dopants with one additional valence electron can be thermally ionized and donate their extra electron to $\mathrm{WO}_{3-x}$ (n-type doping). Similarly, dopants with one fewer/deficient valence electrons can offer an extra hole (p-type doping). These electrons/holes acting as charge carriers can support LSPR in the NIR region, thereby increasing the electrical conductivity. For conducting thin-film technology, the capability to introduce these carriers is necessary, as published for tin-doped indium oxide (ITO), ${ }^{317}$ aluminum-doped zinc oxide (AZO), ${ }^{318}$ indium-doped cadmium oxide (ICO), ${ }^{319}$ and niobium-doped anatase titania. ${ }^{320}$ Hasani et $a .^{321}$ doped CdSe quantum dots with $\mathrm{WO}_{3}$ nanocrystals via wet-synthesis followed by annealing. The optimized CdSe QDdoped $\mathrm{WO}_{3}$ showed an excellent EC performance with a coloration efficiency of $112.3 \mathrm{~cm}^{2} \mathrm{C}^{-1}$. Ni and Ti doping has been reported recently to introduce oxygen vacancies, crystal defects, and nanostructural changes in $\mathrm{WO}_{3}$ for improved EC properties. ${ }^{322,323}$

$\mathrm{Mo}, \mathrm{Cu}$, and $\mathrm{Nb}$ are considered effective sensitizers, increasing the oxygen vacancies and crystal defects. Thus, the absorption peak position of the films shifts to a low wavelength range (blue shift). Moreover, they can also accelerate the reversible switching kinetics of the film. ${ }^{12,324} \mathrm{Al}$ ion doping also adds advantages based on the shape, size, and structural variations. $^{325} \mathrm{Li}$ and $\mathrm{Ru}$ doping successfully enhanced the electrochemical performance, cycle stability, and diffusion coefficient of $\mathrm{WO}_{3}$-based thin films. ${ }^{77,326}$ As the size of nanocrystals decreases, their energy increases, and thus the carriers in the nanocrystals must occupy any confined electronic states. ${ }^{327}$ For robust/stable conductivity, adjacent nanocrystals should be uniform in size to have resonant electron states, and eventually, be narrowly spaced to confirm their quantummechanical overlap. Yao et al. ${ }^{328}$ synthesized Cr-doped $\mathrm{WO}_{3}$ polyhedra by controlling the parameters of RF thermal plasma. The results demonstrated that the 2.5 at\% Cr-doped-layer could reduce the crystal growth rate of $\mathrm{WO}_{3}$ along the $\langle 001\rangle,\langle 010\rangle$, and $\langle 100\rangle$ planes. 

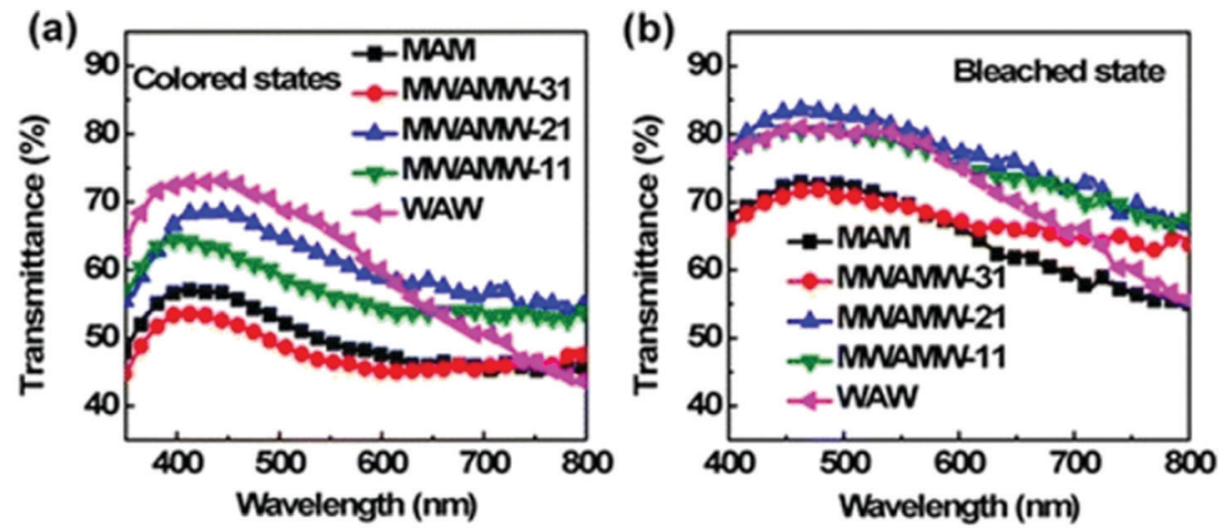

(c)

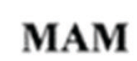

MWAMW-21
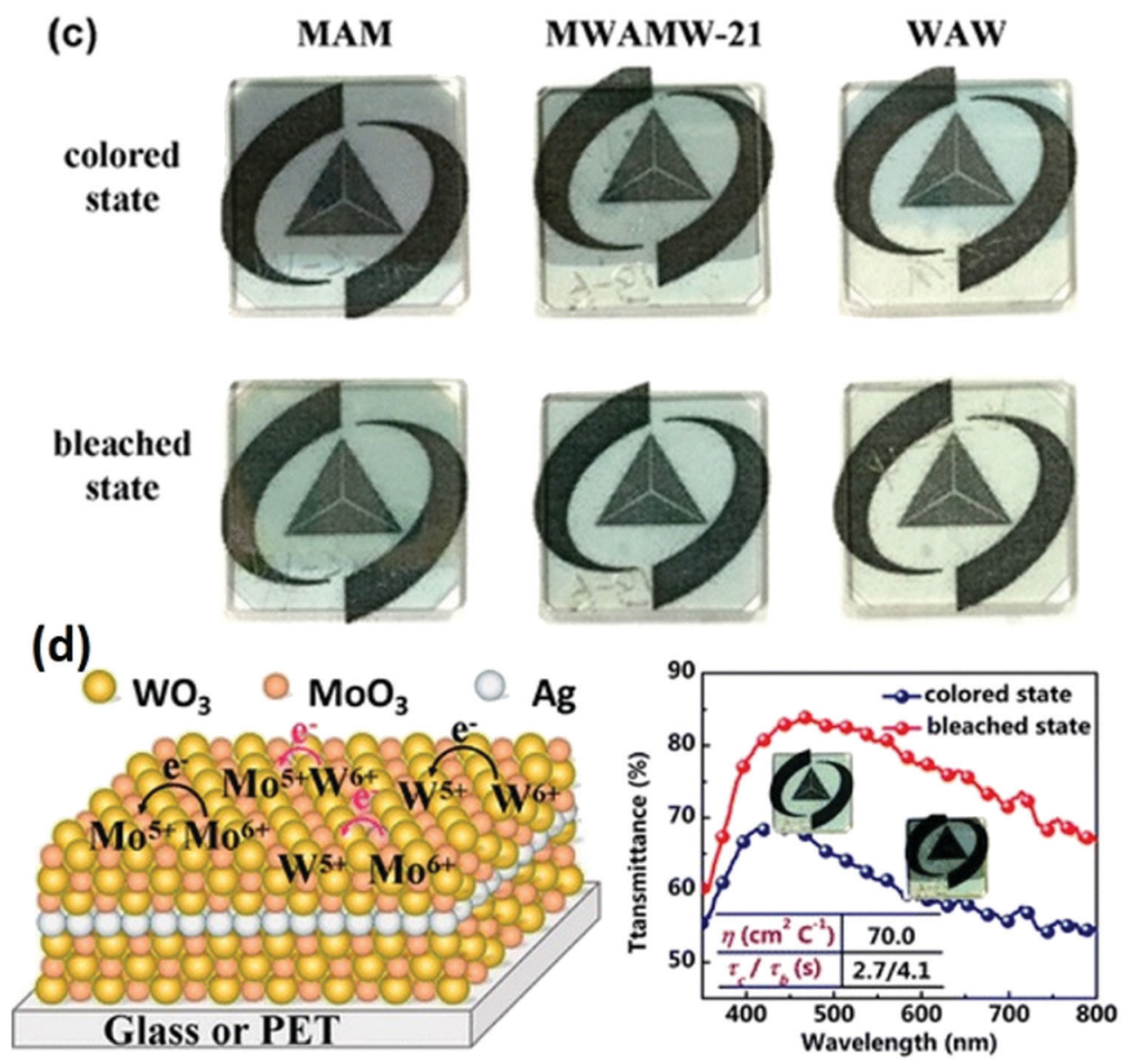

\section{Transparent Conductor + Electrochromism}

Fig. 19 Optical transmittance of MAM, MWAMW, and WAW films in (a) colored and (b) bleached states at potential steps from - 0.50 to $0.55 \mathrm{~V}$ (vs Ag/ $\mathrm{AgCl}$ ) with a $20 \mathrm{~s}$ interval. (c) Pictures of MAM, MWAMW and WAW films in the colored and bleached states. (d) Schematic of MWAMW composite deposited on PET or glass and corresponding color and bleached state optical transmittance. Reproduced with permission. ${ }^{124}$ Copyright@2016, ACS.

Higher carrier concentrations are required for superior conductivity. However, nanocrystals with additional free electrons and holes can also be used as strong reducing/oxidizing agents. Therefore, the electrochemical reactions on the surface can consume the charge carriers, ${ }^{329}$ and the carriers will not participate in electrochemical reactions. Thus, avoiding these reactions of films fabricated using doped nanocrystals is a big challenge! The n-type and p-type doping of CdSe quantum dots using In, Sn, Al, or Ag as dopants have been a research focus, as illustrated in Fig. 20a and b. ${ }^{330,331}$ The critical temperature affecting the doping processes can be summarized as follows: ${ }^{332}$ (i) surface adsorption, (ii) lattice incorporation, (iii) lattice diffusion, and (iv) lattice ejection (Fig. 20a and b). It is noteworthy that each nanocrystal can be switched, but it may 
(a)

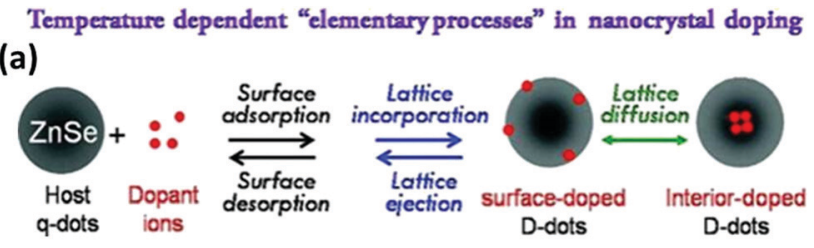

(b)

CdSe $\quad \mathrm{Cd}_{1-x} \mathrm{Mn}_{x} \mathrm{Se}$

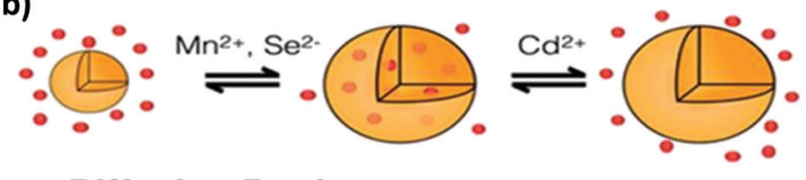

- Diffusion Doping + Cation Exchange -

Fig. 20 (a) Temperature-dependent surface adsorption, lattice incorporation, lattice diffusion, and lattice ejection doping in nanocrystals. ${ }^{332}$ (b) $\mathrm{Mn}^{2+}$ diffusion doping of $\mathrm{CdSe}$ nanocrystals and $\mathrm{Mn}^{2+}$ expulsion from $\mathrm{Cd}_{1-x} \mathrm{Mn}_{x} \mathrm{Se}$ nanocrystals via cation exchange with $\mathrm{Cd}^{2+}{ }^{332}$ Reproduced with permission. Copyright $(\mathbb{C}$ JACS.

be impossible to dope to achieve the desired doping characteristics.

5.3.2. Interstitial doping. Interstitial doping is a principal means for new atoms to be incorporated within the crystal structure because of the atomic vacancies/defects within the lattices. Interstitially doped tungsten oxides, which are known

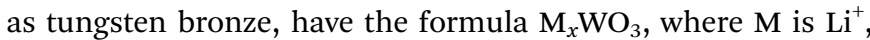
$\mathrm{Na}^{+}, \mathrm{Cs}^{+}$, or $\mathrm{Rb}^{+} .{ }^{333}$ Tungsten bronze materials retain high visible light transmittance, while exhibiting high NIR light absorption, making them ideal candidates for spectrally selective optical devices. A high doping level/degenerate doping in semiconductor metal oxides can effectively modulate the NIR wavelength transmittance through electrochemical charging and discharging of the free electrons responsible for LSPR absorption. ${ }^{41,294}$ It has been found that solar NIR light is the most intense at shorter wavelengths $(700-1300 \mathrm{~nm})$, and thus nanocrystals with LSPR absorption in this spectral range will be ideal for dynamic solar light control. ${ }^{41}$ Due to their outer-d valence electrons, $\mathrm{WO}_{3-x}$ nanoparticles can be ideal candidates with intense visible and NIR light absorption, where their metallic characters exhibit tunable LSPR peaks. ${ }^{139}$ Therefore, interstitially doped/oxygen vacancy-doped nanocrystals can achieve metallic characters with LSPR modulation in the NIR region, besides the substitutionally doped transparent conductive oxides, e.g., ITO/AZO, ${ }^{41,139,294,334}$ used for tuning the LSPR absorption in the IR range (1400-3000 $\mathrm{nm})$. When dopants are incorporated in these nanocrystals, they can emerge as new advances for certain well-known applications and effectively predicted, as found in the literature. ${ }^{294,331,334-338}$

To improve the energy saving proficiencies of EC smart windows, it is necessary to maximize the plasma resonance displacement and modulations of NIR rays. For example, in ITO, a high doping concentration shifts its LSPR peak to the high frequency spectrum. In contrast, a decrease in the nanocrystal size to $\sim 2-5 \mathrm{~nm}$ in accordance with an increase in optical modulation remains enigmatic. Smaller nanocrystals have a higher surface charge density, which facilitates high capacitive behavior and allows more electrons to be injected. Therefore, smaller size and highly doped nanocrystals will exhibit improved EC performances in the desired multifunctional EC smart windows. Because of the impurity atom doping and higher formation energy of defective nanocrystals, nanocrystals tend to expel dopants during annealing to minimize their energy and undergo self-purification/healing (Fig. 20). ${ }^{339}$ It was found that surface adsorption and diffusion ${ }^{339}$ can be influenced by three main factors: (i) surface morphology, (ii) nanocrystal shape, and (iii) surfactants in the growth solution. Based on the kinetic control, dopants can be incorporated in the host nanocrystals if they can bind and reside on the host crystal surface. Diffusion of impurities into the host nanocrystals may occur on the surface of nanocrystals. It was also found that nanocrystals contain active surface sites and the binding energy of the impurities adsorbed on the nanocrystals determine their residence time. ${ }^{339}$ The diffusion doping strategy is based on incorporating relatively incompatible anions/cations in semiconductors, which is driven by thermodynamic and high-temperature cation randomization. For, example $\mathrm{Cd}_{1-x} \mathrm{Mn}_{x}$ Se has been studied (Fig. 20b). ${ }^{340}$ The slow diffusion kinetics of $\mathrm{Mn}^{2+}$ ions and high-temperature treatment possibly accelerate their diffusion, although it is associated with selfpurification due to the diffusion of $\mathrm{Mn}^{2+}$ ions out from the nanocrystals. $^{332}$

It is worth noting that only effective doping that successfully incorporates dopants in the host crystal lattice rather than adsorbed on its surface can alter the characteristics properties of nanocrystals; however, plasmonic nanocrystals are quite interesting in this regard. ${ }^{314,340} \mathrm{Au}$ and $\mathrm{Ag}$ are believed to have strong surface-enhanced Raman spectroscopy (SERS) performance. They have no signals upon Raman scattering but can produce strong LSPR under irradiation of light. Because $\mathrm{Au}$ couples with Ag substrates, and then non-noble metals, metal oxides, graphene, silicon-metal and metal-tellurides are also considered to become a new type of SERS materials due to the charge transfer mechanism. Liu and coworkers ${ }^{341}$ reported that $\mathrm{TiO}_{2}$ and $\mathrm{WO}_{3-x}$ do not have free electrons because of their wide bandgaps, indicating that these semiconductors will not show the LSPR effect. However, their conductivity can be greatly enhanced due to the introduction of dopants into their large vacancy/defect sites, given that the dopant materials have the LSPR effect due to the free electrons found in their d-orbital, as shown in Fig. 4b and 21. However, the SERS induced due to the LSPR in $\mathrm{TiO}_{2-x}$ and $\mathrm{WO}_{3-x}$ cannot be generated due to the presence of oxygen vacancies and crystal defects. This can be attributed to the difference in excitation wavelength between NIR and SERS, which are found in the visible region. However, the conductivity (charge transfers) greatly increases due to the vacancy-doped features between the substrates and the adsorbed molecules. Therefore, if the LSPRs of vacancy-rich materials can be adjusted from the NIR region to the visible region (near the SERS excitation wavelength), then due to the charge transfer process, their SERS activity can be further improved. The high density and oriented $\mathrm{W}_{18} \mathrm{O}_{49}$ ultrafine nanowire bundles with an abundance of oxygen vacancies 

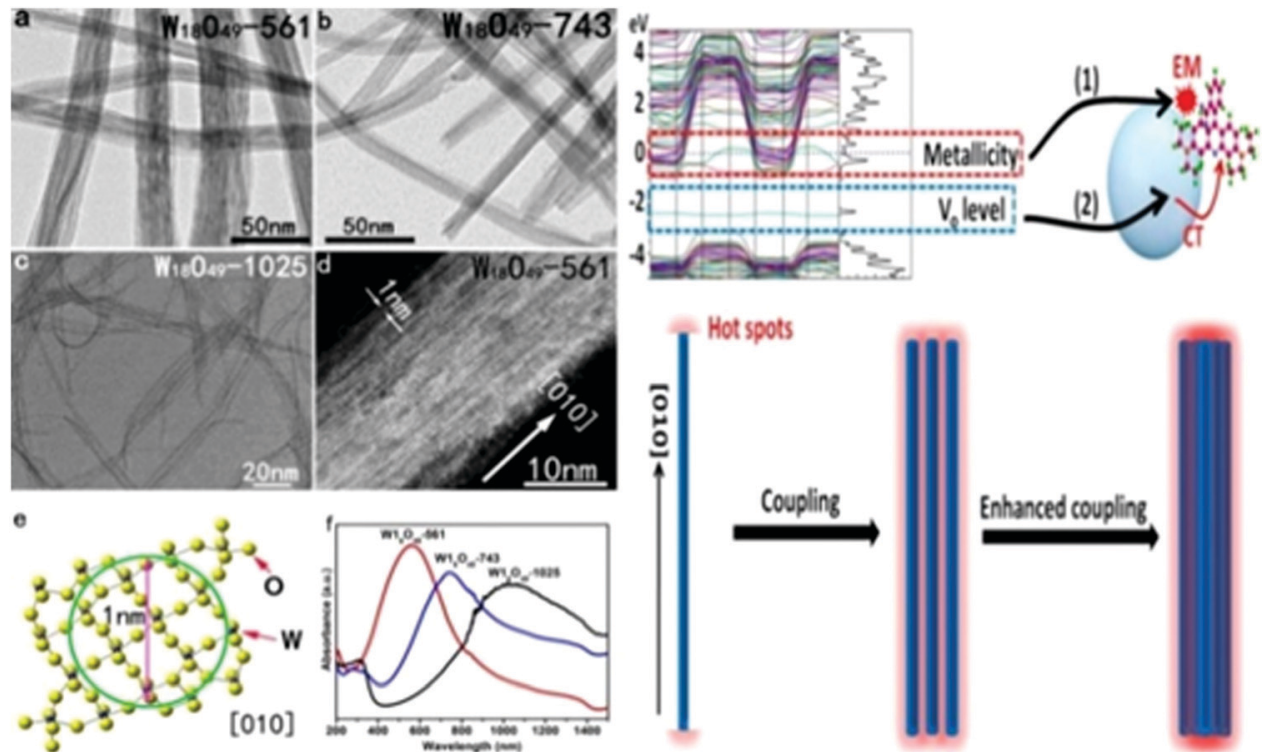

Fig. 21 (a-c) TEM images of $\mathrm{W}_{18} \mathrm{O}_{49}-561, \mathrm{~W}_{18} \mathrm{O}_{49}-743$, and $\mathrm{W}_{18} \mathrm{O}_{49}-1025$, distinguished due to various in their LSPR peaks. (d) HRTEM image of $\mathrm{W}_{18} \mathrm{O}_{49}-561$. (e) Cross-section of a $1.0 \mathrm{~nm}$ nanowire inside of one $\mathrm{W}_{18} \mathrm{O}_{49}$ unit cell oriented along the [010] direction. (f) UV-visible-NIR absorption spectra of the $\mathrm{W}_{18} \mathrm{O}_{49}{ }^{-}$ 561, $\mathrm{W}_{18} \mathrm{O}_{49}-743$, and $\mathrm{W}_{18} \mathrm{O}_{49}-1025$. (left) Top: Band structure of $\mathrm{W}_{18} \mathrm{O}_{49}$ and the two possible SERS enhancement ways in $\mathrm{W}_{18} \mathrm{O}_{49}$ : (1) $\mathrm{EM}$ and (2) CT. Bottom: LSPR coupling between nanowires. Adopted from ref.341 Copyright $(\mathrm{C} 2018$, ACS.

and defects have strong LSPR due to the charge transfer and electromagnetic enhancement factors. With an increase in thickness due to the aggregation of the nanowires, the absorption peaks gradually change from the NIR to visible region (Fig. 21). However, the XRD peaks remain almost the same, which was concluded to be due to electromagnetic oscillations found in the highly thin $\mathrm{W}_{18} \mathrm{O}_{49}$ nanowire bundles. These pioneering results may reveal SERS properties. ${ }^{341}$ The SERS enhancement mechanism can be attributed to the synergistic effect of the LSPR-SERS coupling that occurred among the ultrathin oriented nanowires, defects, and oxygen vacancy, which significantly improves the charge transfer process. ${ }^{341}$

\subsection{Plasmonic surface-decorated $\mathrm{WO}_{2.72}$ composites}

Plasmonic nanocrystal-modified interfaces significantly affect the optical absorption properties of nanomaterials. Noble metals nanocatalysts, such as $\mathrm{Au}, \mathrm{Pd}, \mathrm{Pt}, \mathrm{Ag}, \mathrm{Rh}$, and $\mathrm{Cu}$, are promising for improving the optical properties via LSPR effective modulations and Schottky junction formation. This is because the difference in the work functions of noble metals and the semiconductor metal oxide $\left(\mathrm{WO}_{2.72}\right)$ induce electronic sensitization, affecting their electrical properties. The rectifying Schottky barrier at the interface enhances the electrical transport properties. Thus, a higher work function and Schottky junction formation can significantly facilitate the separation of photogenerated electrons and holes. Ordered-shaped plasmonic nanocrystals such as nanorods (1D), sheets (2D), cubes, and triangular prisms (3D) possess sharp edges and corners, and hence exhibit excellent LSPR and considerable electromagnetic field enhancement.

Plasmonic-mediated nanocrystals can be classified into four categories as follows: (i) isotropic/bulk, (ii) assembled, (iii) responsive, and (iv) interfacial plasmonic nanocrystals. In bulk/isotropic plasmonic nanocrystals, the nanocrystal composite is monodispersed throughout the matrix compared to the bulk in a medium. The dielectric matrix may cause an insulating barrier between the plasmonic nanocrystal candidates or may also contribute to their optoelectronic properties. In assembled nanocrystals, the plasmonic component species are arranged into groups, clusters or certain anisotropic areas in the dielectric matrix. This self-assembly causes the nanoparticles to acquire strong plasmonic coupling due to the formation of nanojunctions and their collective optical properties. New research is devoted to reconfiguring the capability of plasmonic nanocrystals to modulate external stimuli that trigger a high optical response. Thus, the responsive plasmonic nanocrystals can acquire tunable and unique optical functionalities. ${ }^{10}$ It is noteworthy that the gaps between the nanocrystals often have an impact on their optical properties. The interfacial plasmonic nanocrystals connect the interfaces of the metal-dielectric (M-D), dielectric-dielectric (D-D) or dielectric-metal-dielectric (D-M-D). These composite architectures are frequently 2D/3D structures. Electron injection in $2 \mathrm{D} /$ 3D plasmonic nanocrystal composites results in a shift towards the high-frequency region and optical modulation in the NIR region of the solar spectrum. Plasmonic composites are specially engineered to acquire optoelectronic properties different from their bulk components. The blend symmetries of plasmonic-incorporated particles can be understood based on Fig. 22, and detailed explanations can be found in the chemical review published by Su-Wen Hsu et al. ${ }^{342}$

The Vis-band optical modulation of $\mathrm{WO}_{3-x}$ can be shifted towards the NIR wavelength due to the coupling of traditional metals/cation intercalation, resulting in NIR-LSPR extinction. 
i. Bulk pNCs
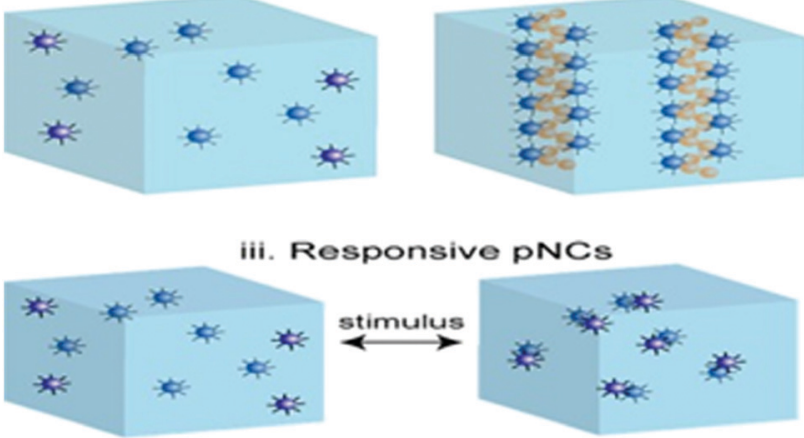

iv. Interfacial pNCs

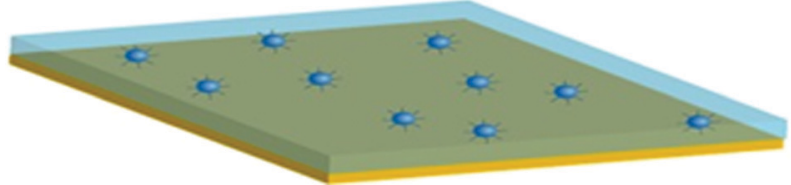

Fig. 22 Schematic of the various classes of $p N C s$. Reproduced from ref. 342 Copyright@2018, ACS.

Thus, the surface decorating/synthetic doping of noble metals in $\mathrm{WO}_{3-x}$ nanocrystals should be capable of independent modulation of spectral bands in both the Vis and NIR regions within a single-component functionalized film. Thus functionalized $\mathrm{WO}_{3-x}$ can be capable of dual-mode optical modulation as a nonradiative material to achieve multifunctional smart windows for controlling visible light transmittance, photothermal conversion of NIR solar loads, and also eliminating microorganisms living on windows. ${ }^{67,293,343,344}$ The growth of plasmonic nanocrystals on metal oxide layers can support the tunable LSPR, which will interact with the solar spectrum to shift the plasmon resonance for color changes. ${ }^{42}$ Moreover, Li-ion intercalated $\mathrm{WO}_{3-x}$ can be used for energy storage applications, such as supercapacitors and batteries. ${ }^{133,345}$ Improving the slow diffusion kinetics of $\mathrm{Li}$ ions and the conductivity of bulk $\mathrm{WO}_{3-x}$ can improve the charge capacity and charging capability of $\mathrm{WO}_{3-x}$-based electrodes. Assuming that surface-modified $\mathrm{WO}_{3-x}$ can be charged, its charge capacity is almost equivalent to that of lithium insertion reactions in ultra-small particles of about $10 \mathrm{~nm} .{ }^{42,346}$ Besides decreasing the size of $\mathrm{WO}_{3-x}$, doping can also improve the electrochemical properties of $\mathrm{WO}_{3-x}$ electrodes (see Section 5.3) to boost their inherent low conductivity. Furthermore, surface-decorated nanocrystals with a diameter of $<10 \mathrm{~nm}$ can support LSPR, which can be tuned through the Vis to NIR region by altering the size, shape, composition, and Fermi energy level of the nanocrystals, as reported in the literature. ${ }^{67,347,348} \mathrm{Au}, \mathrm{Ag}$, and Pd nanoparticles may exhibit a strong, robust, stable, and tunable LSPR absorption band at both visible and NIR absorption wavelengths. ${ }^{69,349} \mathrm{Au}$ and Pd nanoparticles with a spherical shape and size of $\sim 4-6 \mathrm{~nm}$ were deposited on the surface active sites of $\mathrm{WO}_{3}$ nanowire bundles in $\mathrm{HAuCl}_{4}$ and $\mathrm{PdCl}_{2}$ solutions with additional oxidation ${ }^{67,149,350}$ (Fig. 23). In this scenario, the absorbed NIR light could subsequently be
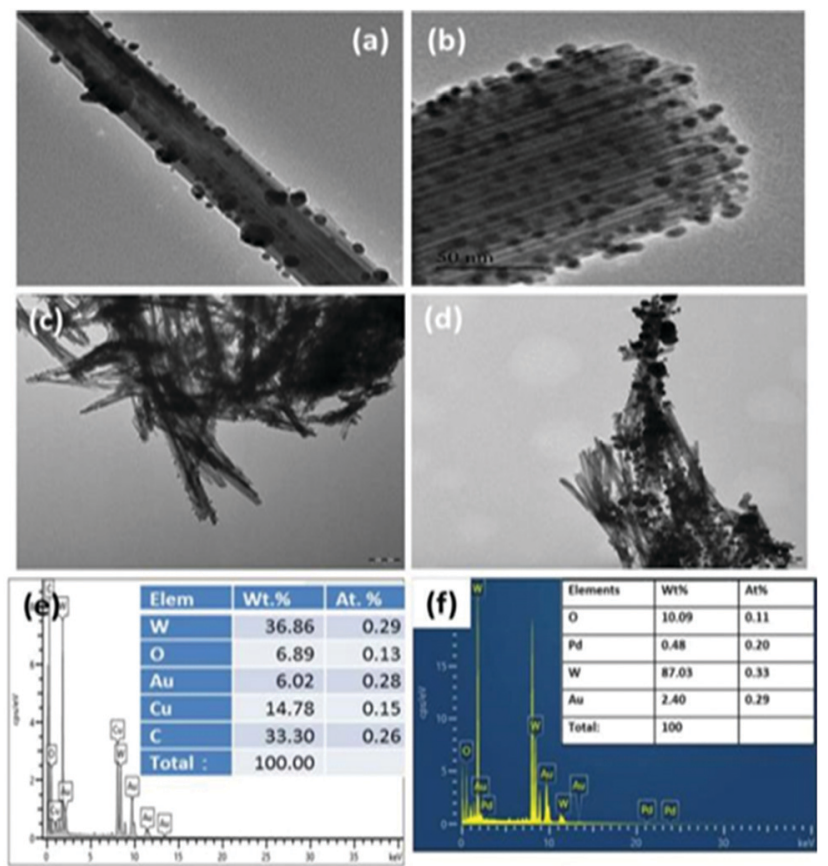

Fig. $23(a-d)$ TEM and HRTEM images displaying $W_{3-x}$ nanorods: nanowire assembly decorated with $\mathrm{Au}$ ( $\mathrm{a}$ and b), $\mathrm{Pd}$ (c), and (d) bimetal $\mathrm{Au}, \mathrm{Pd} \mathrm{NP}$-decorated $\mathrm{WO}_{3}$. (e and f) Corresponding EDS elemental mapping. Reproduced with permission. ${ }^{149}$ Copyright $(\mathrm{C}$ Elsevier.

converted to heat energy due to the electron-phonon/phonon-phonon interactions. Au NP-decorated $\mathrm{WO}_{3}$ may enhance the catalytic and photocatalytic activity and exploit the LSPR in $\mathrm{Au}$ NPs. The extensive work functions of $\Phi(\mathrm{Au})=5.1 \mathrm{eV}, \Phi(\mathrm{Pd})=$ $5.5 \mathrm{eV}$, and $\Phi\left(\mathrm{WO}_{3}\right)=4.8 \mathrm{eV}$ may induce electronic sensitization and trap photo-excited electrons. The LSPR in gold-decorated $\mathrm{WO}_{3-x}$ has been applied in photothermal ablation therapy, ${ }^{351}$ biological sensors, ${ }^{352,353}$ gas sensors, ${ }^{354-356}$ and optical detection. $^{357}$

Xu et al. $^{293}$ fabricated an EC-photothermal dual-band composite film of plasmonic Au anchored with 3D honeycomb-like $\mathrm{WO}_{3}$, as shown in Fig. 24a-d. The obtained composite film was tested for the optical transmission and photothermal conversion of visible and NIR light and reducing the attack of microorganisms (Fig. 24e). The Au LSPR and broadband nonradiative plasmon decay are assumed to be tunable as an electric field on the surface of the $\mathrm{WO}_{3}$ substrate. The photothermal conversion was attained in a colored state, which is ascribed to the coupling of traditional visible-band optical switching with the NIR-LSPR extinction, as given in Fig. $24 \mathrm{f}$ and g. The as-prepared EC and photothermal films were also examined and proven to be effective in reducing the risk of microorganisms such as E. coli bacteria. Therefore, photothermal EC film-coated glass as an advanced multi-functional smart sterile window has broad application prospects.

Moreover, Ag nanoparticles were distributed isotropically within the bulk polytetrafluoroethylene (PTFE) matrix through direct current (DC) or co-sputtering of Ag and PTFE to leverage the LSPR extension of the $\mathrm{Ag}$ nanoparticles in the visible 

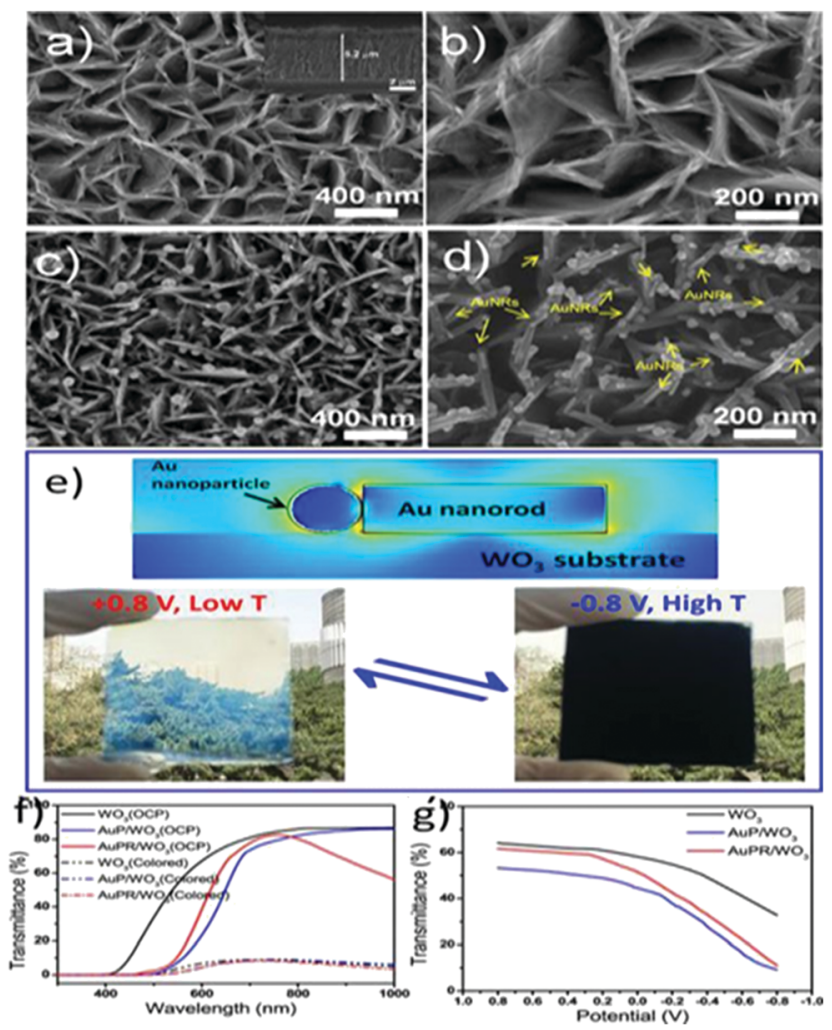

Fig. 24 SEM images (a) top view and (b) magnified view of a blank $\mathrm{WO}_{3}$ film; (c) top view of Au nanoparticle-decorated $\mathrm{WO}_{3}$ film; and (d) top view of Au nanoparticle- and nanorod-decorated $\mathrm{WO}_{3}$ film. (e) Schematic of Au nanoparticle-attached nanorods, and digital photographs of the AuPR/ $\mathrm{WO}_{3}$ film on FTO in the bleached and colored state. (f) Transmission spectra of the $\mathrm{WO}_{3}, \mathrm{AuP} / \mathrm{WO}_{3}$, and AuPR/WO film-coated FTO slides at OCP and colored states. (g) Relationship of transmittance at $915 \mathrm{~nm}$ with an applied electrochemical potential at a scan rate of $10 \mathrm{mV} \mathrm{s}^{-1}$. Reproduced with permission from ref. 293 Copyright@2018, ACS

range. ${ }^{342,358}$ The deposition process is important for developing flexible substrates that utilize matrix/nano-inks and target nanoparticles with a very small size. The plasmonic nanoparticle-based composite-generated film on the flexible substrates not only selectively controls the solar spectrum as an EC device but also has retrofit installation features. ${ }^{359}$ During in situ synthesis, the small size and morphology, and aspect ratio of plasmonic nanoparticles can cause impediments. Accordingly, plasmonic nanoparticle-polymer blends can successfully overcome this disadvantage via the incorporation of a polymer matrix in the already produced metal nanoparticles. Therefore, each material component of the plasmonic nanocrystals/composite can be synthesized independently and combined with the prospective polymer blend to generate a multifunctional nanocomposite. ${ }^{360-362}$

Nanoscale $\mathrm{WO}_{3-x}$ particle decoration is advantageous for the fabrication of plasmonic nanocrystal-composites, which prevents undesirable aggregation or may be beneficial for phase separation. Similar to $\mathrm{NiO}, \mathrm{WO}_{3}, \mathrm{TiO}_{2}$, and $\mathrm{VO}_{2}$, the color and optical transmittance can be tailored by external stimuli, including an electrical field, light, and temperature. ${ }^{10}$
In these scenarios, the nanocrystal components are grafted with polymer templates, which are miscible in a polymer matrix, i.e., dispersion of fullerenes in polystyrene. ${ }^{363}$ Similarly, they can also be grafted through the large gyration radius of linear polymers, i.e., polyethylene nanoparticles in polystyrene, ${ }^{363}$ and/or tethered with soft/hard templates having a high surface area, which endows the highest possible stability to the nanocrystal composite-polymer templates. ${ }^{364} \mathrm{Au}$ nanorods were grafted with polystyrene polymer, and then deposited as a thin film. ${ }^{365}$ The thin film showed an optical response at $780 \mathrm{~nm}$, which is reliable with the excitation of the longitudinal dipolar LSPR modes. The LSPR resonance function showed a blue shift in the optical absorption, resulting from the long linear-polymer templates and the side-by-side assembly of the aligned nanorods. In addition, the plasmonic EC film-based electrodes with nanocrystals in a disordered packing ${ }^{123}$ generated open network channels, which increased the surface area and could be easily accessed by the electrolyte. In this area of research, the Milliron team demonstrated the fabrication of porous films and the effect of the nanopore sizes obtained by using amphiphilic block copolymer micelles to bind ligandstripped ITO nanocrystal architectures into an ordered/more alignment conductive mesoporous network. ${ }^{366}$ The mesoporosity was controlled by altering the molecular weights of the hydrophilic/hydrophobic block polymers, with the pore size in the range of $29-37 \mathrm{~nm}$ and thicknesses of $9-13 \mathrm{~nm} .{ }^{366}$ The ordered mesoporous structures resulted from the interaction between the ligand-stripped nanostructure surface and hydrophilic block-copolymers. ${ }^{367}$ Increasing porosity increases the speed of ion transport and switching through the surface of the nanocrystals. $^{347,367}$

In addition, the controllable size, quality, and assembly structure of plasmonic nanocomposites can be integrated into traditional multi-functional smart windows, where each component can be activated independently for specific functions. The independent modulation of visible and NIR light will stimulate the window to function in hot/cold climates to respond perfectly to changes in weather conditions. Recent research relies on plasmonic nanocomposite architectures for visible and NIR-selective transmission, enabling dualband functionality to meet the challenges of traditional smart windows. ${ }^{122,123}$ Dahlman et al. ${ }^{42}$ fabricated a single-component colloidal aliovalent niobium-doped anatase $\mathrm{TiO}_{2}$ nanocrystal film for dual-band optical modulation. The $\mathrm{Nb}-\mathrm{TiO}_{2}$-deposited film showed LSPR in the NIR region and could modulate light with capacitive charging. A variation in the composition, size, shape, and doping in the $\mathrm{TiO}_{2}$ nanocrystals greatly affected the efficiency of the EC film. The anatase $\mathrm{TiO}_{2}$ has low electrical conductivity and slow ion diffusion, which was improved by composite formation and impurity doping.

Moreover, multifunctional integrated devices have received tremendous research interest due to their low cost, lightweight, flexibility, and for monitoring health and the environment. Recent research achievements such as gas sensors, strain sensors, and photodetectors have been reported in various self-powered multifunctional integrated systems, thus reducing 
$\mathbf{a}$
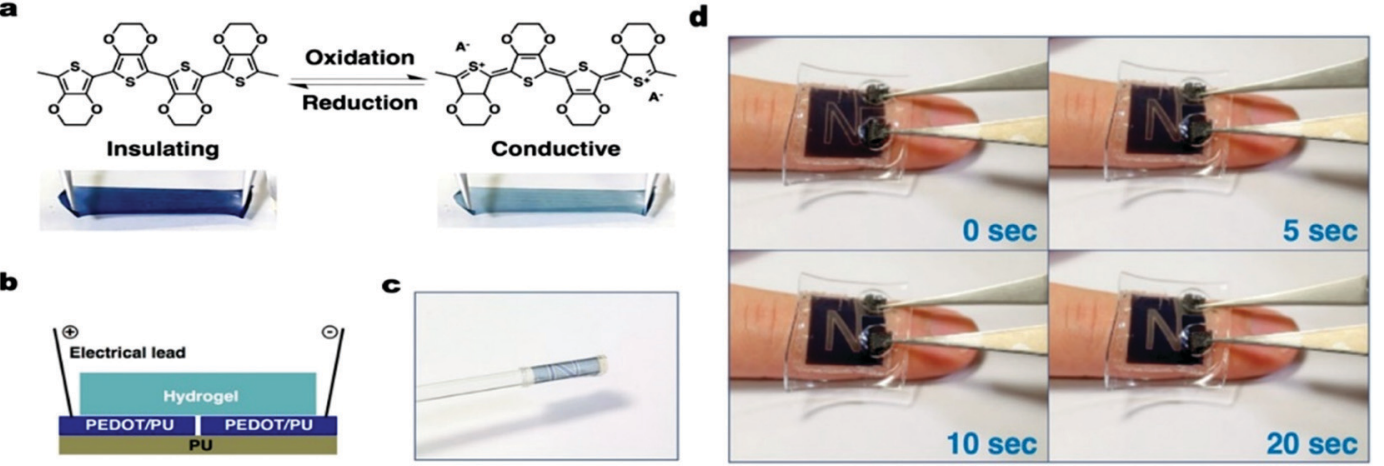

Fig. 25 Stretchable EC film of PEDOT/PU. (a) Molecular structures of PEDOT and photographs of the stretched EC film of a PEDOT/PU composite in the reduced and oxidized states. (b) Schematic illustration of an EC film/hydrogel hybrid. (c) EC film/hydrogel hybrid wrapped around a glass rod and human finger. (d) Color change in the EC film/hydrogel hybrid device placed on a human finger. Reproduced with permission. ${ }^{375}$ Copyright@2017, ACS.

energy cost by cooling, heating, and lighting. ${ }^{68,293,368}$ For example, flexible supercapacitors have been reported together with photodetectors based on CdSe nanowires, ${ }^{369} \mathrm{Co}_{2} \mathrm{O}_{3}$ nanowires on Ni fibers with graphene, ${ }^{370} \mathrm{TiO}_{2}$ nanoparticles, ${ }^{371}$ and $\mathrm{TiO}_{2} /$ MXene heterostructures ${ }^{372}$ deposited on different substrates. The resultant flexible all-solid-state integrated systems exhibit energy-storage performances together with an excellent response to white light. Yun et al. ${ }^{373}$ fabricated a stretchable $\mathrm{SiC}$ gas sensor for $\mathrm{NO}_{2}$ on an Ecoflex substrate, which could detect gas with a sensitivity of about $50 \mathrm{~min}$ after being charged. Moreover, flexible, self-powered devices have been reported, which are sensitive to human touch or motion. ${ }^{374}$ Kai et $a l .{ }^{375}$ synthesized a stretchable conductive composite by mixing poly(3,4-ethylene dioxythiophene):p-toluene sulfonic acid (PEDOT:PTS) mixed with polyurethane (PU). The film fabricated by spin coating showed dynamic electrochromism in a hydrogel without supplying an external voltage, as given in Fig. 25. The stretchable PEDOT/PU film is suitable for a wearable display device; hence, it can be ultimate proof for the multifunctional features of wearable-to-self-powered with sensory smart window applications.

\section{State-of-the-art EC devices: principal factors and composition}

\subsection{Principle factors}

In an exemplary EC device (Fig. 1), cathode EC materials exhibit their inherent optical properties without an applied electrical potential, and the electrolyte ions (cations and anions) are uniformly dispersed in the device. When an electric potential is applied, the EC material captures the injected electrons, and thus the material gets reduced and then ionized, with a change in optical property to the "switching" mode. Meanwhile, the cations from the electrolyte move toward the opposite electrode and gather in the area of negatively ionized EC materials to balance the charge near the cathode. Also, the anions from the electrolyte enter the counter electrode to balance the charge and are stabilized by oxidization. Thus, fully reversible redox reactions occur with an appropriate reverse voltage. Therefore, the performance of EC devices depends not only on the electrochemical reaction of heterogeneous electron transfer between the electrode and the EC film and/or ion storage film, but also on the homogeneous ion(s) transfer through the EC layer(s), the ion-transport layer, and the ion-storage layer. Thus, the EC performance can be optimized as follows: (i) by increasing the surface activity between electrodes and the EC film and/ or ion storage film; (ii) increasing the electronic conductivity of electrodes and/or the ionic conductivity of electrolytes; and (iii) advancement to EC materials.

The EC material films are the key components defining the EC device performance, where the reversible optical modulation occurs under a suitable voltage. The dynamic windows consisting of $\mathrm{WO}_{3-x}$ can be electrochemically/electronically color/bleached with the insertion of a tiny electric potential (1-3 V) to dynamically control the inlet/exit of thermal loads and daylight via smart windows of buildings, vehicles, aircraft, etc.

EC devices can be (i) single EC and (ii) dual/multiple EC.

(i) Single EC devices are developed from one EC material coated as a thin layer. This type comprises materials with alternative one coloured state and one bleached state, which is useful in absorption/transmission-type devices. These devices may have fewer redox states compared to that composed of more than one EC active species.

(ii) Dual/multiple EC devices consist of two/more active EC materials triggered for multifunctional features in a single platform (Fig. 1). However, two/more active materials on the same platform can cause system complexity. Nevertheless, they have significant advantages in the possibility of multiple color changes at small applied potentials and multifunction. Dualfunction devices designed with cathode and anode EC materials in one platform (Fig. 1) are considered to be more efficient, powerful, and therefore can save a tremendous amount of energy.

Based on their operation, EC devices can be transmissive type, which operates on the principle of optical transmission, and reflective type, regulating the optical reflectance. Further progress remains to be achieved in developing more efficient multifunctional EC devices with different capabilities in a 
single platform. The key parameters used for evaluating the EC device performance are as follows:

6.1.1 Coloration efficiency

6.1.2 Switching time

6.1.3 Cycling life

6.1.4 Device stability and durability

6.1.1. Coloration efficiency. The change in optical modulation (optical density) occurring per unit injected charge is known as the coloration efficiency (CE), which has an impact on selecting the EC material and device performance. It can be expressed as follows:

$$
\mathrm{CE}=\Delta \mathrm{OD} / Q=\log \left[T_{\text {bleached }}(\lambda) / T_{\text {colored }}(\lambda)\right] / Q
$$

where $T_{\text {bleached }}$ and $T_{\text {colored }}$ are the transmittance in the bleached and colored states, respectively. The coupling of electrochromism and energy storage involves a high CE $\left(\mathrm{cm}^{2}\right.$ $\mathrm{C}^{-1}$ ) and fast switching speed with the supply of low power for high optical modulation and charge density. Generally, an EC device having a high coloration efficiency will be more durable and switch faster. ${ }^{79}$

6.1.2. Switching time. Switching time is the response time required to achieve switching efficiency between the transparent and different colored states. The switching speed depends on the (i) conductivity of the electrodes, (ii) ionic conductivity of the electrolyte, (iii) diffusion of ions in the thin films, (iv) magnitude of the applied potential, (v) film thickness, (vi) microstructure size, and (vii) surface morphology.

6.1.3. Cycling life. The cycle life refers to repeatable cycles between the colored state and bleached state under an applied voltage. Due to repeatable cycles, device failure may occur, such as solid-phase changes, and/or side reactions may occur, and thus the lifetime of thin layers decreases with changes in the electrode composition.

6.1.4. Device stability and durability. The stability towards redox reactions generally limits the utility of EC materials. For practical impact, EC smart windows should sustain steady switching after thousands $\left(10^{4}-10^{6}\right)$ of repeatable cycles, which is a key obstacle for the perspective development of nextgeneration EC devices. In addition to long-term switching stability, device durability is also a key parameter. Moreover, the device should remain stable in an environment that is very cold (below $-20{ }^{\circ} \mathrm{C}$ ) or very hot (above $80{ }^{\circ} \mathrm{C}$ ). Moreover, EC devices are generally sealed to avoid the adverse consequences of humidity, oxygen, air, and water in the external environment. Finally, the given appropriate voltage schemes and combination of electroactive materials candidates inside the EC devices are beneficial for promising EC properties.

\subsection{Device configuration}

Optical transparency and mechanical flexibility have become new developments in the next generation of flat panel electronics, enabling new technologies, including EC windows, (e)skin, wearable optoelectronics, and rolled-up displays. ${ }^{376,377}$ A traditional EC device is composed of a 4-5 layer configuration, and nanomaterials are widely used in each layer to achieve better performances and reliability. The recent advances on EC materials (inorganic/organic) have been reviewed by Yang et al., ${ }^{84}$ Rai et al., ${ }^{87}$ Kim and Yang, ${ }^{10}$ and Eh et al., ${ }^{196}$ and the state-of-the-art approach of EC materials towards multifunctional EC smart-windows by Cai et al. ${ }^{83}$ and recent progress in smart windows based on electro-, thermo-, mechano-, and photochromics is summarized by Ke et al. ${ }^{8}$ The more recent development in EC materials triggered in each layer to build the next-generation smart windows for "zeroenergy buildings" is summarized in this review, as illustrated in Fig. 26.

6.2.1 Transparent conductive substrates

6.2.2 Ion conducting electrolyte

6.2.3 Ion storage/conductive oxide counter electrodes (anode)

6.2.4 EC material $\left(\mathrm{WO}_{2.72}\right)$ cathode

6.2.1. Transparent conductive substrates. For EC devices, the ideal electrode materials not only need to possess tremendously high conductivity, but also excellent transparency and high chemical/electrochemical corrosion resistance capabilities. The CVD, PVD, PLD, spray coating, spin coating, inkjet printing solution casting, and dip coating methods have been considered to be the best techniques for forming transparent thin films. ${ }^{378}$ The transparent conducting electrode materials used in EC devices will be summarized as:

(i) Doped metal oxides. For EC glasses, thin transparent conducting films consisting of doped metal oxides are generally employed as conductive electrode supporting substrates. Common examples include $\mathrm{In}_{2} \mathrm{O}_{3}: \mathrm{Sn}$ (ITO), $\mathrm{SnO}_{2}: \mathrm{F}$ (FTO), $\mathrm{SnO}_{2}: \mathrm{Sb}$ (ATO), $\mathrm{In}_{2} \mathrm{O}_{3}: \mathrm{Zn}$ (IZO), ZnO:Al (AZO), ZnO:Si (SZO), ZnO:Ga (GZO), ZnO:B (BZO), ZnO:F (FZO), ZnO:Nb, ZnO:Sc, and $\mathrm{SnO}_{2}: \mathrm{Ta}^{5,379}$ All these co-doped oxides have wide bandgaps and are transparent in both the visible and NIR light spectrum. They can develop several bilayer architectures with a combined

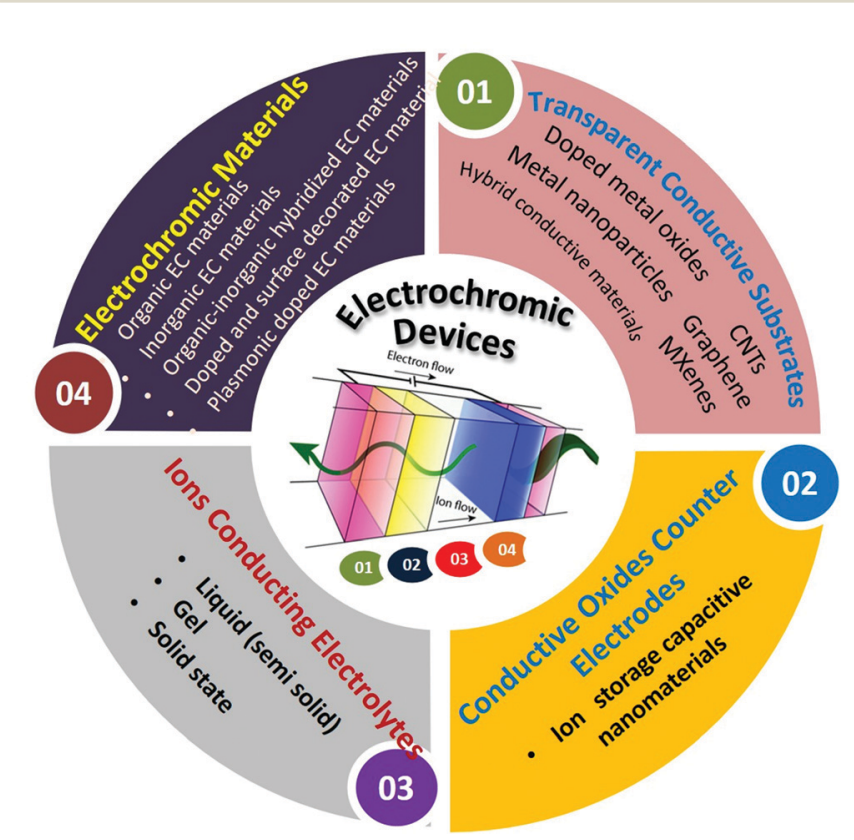

Fig. 26 Schematic illustration of the composition of EC devices. 
resistivity of about $\sim 1 \times 10^{-4}-10^{-3} \Omega \mathrm{cm}^{-1}$, expected thickness of up to $\sim 300 \mathrm{~nm}$, and can be reduced to a certain adequate value with excellent durability. With the arrival of flat-panel display devices, ITO became the most commonly used transparent conductive oxide material for transparent electrodes; ${ }^{378,379}$ however, it is still not available on a large scale for EC smart windows. All these oxides are transparent in the solar spectrum.

The large-scale integration of traditional wearable electrochromic devices requires the development of flexible, stretchable, and deformable substrates. ${ }^{196,372,375,377,380}$ Generally, polymer, CNTs, graphene, MXenes, metal film, plastic, carbon fibre, silicone, hydrogels, and paper are used as supporting substrates due to their outstanding mechanical flexibility and physiochemical stability (resistivity, heat, good acid-base corrosion resistance, etc.). ${ }^{380}$ ITO is cost-expensive and brittle. Thus, conductive coatings on the surface of flexible EC substrates may be destroyed and cracked due to excessive stretching and bending processes at a lower strain of 2-3\%. Concerns have also been raised over the limited indium source, stringent deposition conditions, and consequently high costs. ${ }^{381}$ It is unstable in acidic/basic environments, which leads to a shortened device lifetime. Moreover, ITO films have a high refraction index, and thus can cause unwanted reflections. ${ }^{381}$

Polyethylene terephthalate (PET) supporting substrate is a commonly used bendable electrode material due to its easy manufacture, low cost, excellent flexibility, super transparency ( $>85 \%$ ), and constancy in weak acids-bases. ${ }^{382,383}$ Thermal evaporation and photolithography can be applied for deposition on PET substrates; however, its brittleness and processing at high-temperature $\left(150{ }^{\circ} \mathrm{C}\right)$ limit its use in flexible EC devices. Therefore, it is difficult to distribute the functional electrode materials and sensing materials on PET substrates through direct chemical preparation. ITO- and FTO-coated glass substrates have been replaced by PET/polymers (polyimide), and because of their excellent conductivity, these materials improve the cycle stability and colour efficiency, and hence are suitable for use in EC devices (Fig. 1). ${ }^{37}$ Polyimide (PI) sustains a high temperature $\left(400{ }^{\circ} \mathrm{C}<3 \%\right)$ without affecting wearability and transparency. ${ }^{380}$ Gas-phase and wet-chemical methods can be used to deposit materials on PI substrates, which demonstrates that the active materials have strong adhesion with the substrates, thereby improving their electrochemical properties. Moreover, ultra-thin silicon films $(5-10 \mathrm{~m})$ and mica plates are also a good choice for EC material growth and device manufacturing due to their high thermal stability $\left(>600{ }^{\circ} \mathrm{C}\right)$. Graphite, carbon paper and MXenes have attracted great attention because of their foldable, bendable, and rollable properties. Flexible substrate-based electrodes in EC devices have retroflected installation ability on existing windows, which significantly reduces costs compared to new installations. However, for large-area practical applications, the development of flexible EC devices at present is in its infancy. For example, in advanced flexible EC devices, the effect of UV light on polyester/PET foils can cause degradation and will affect the lifetime of EC windows. To avoid the brittleness of FTO and ITO glass, some flexible transparent substrates such as graphene, ${ }^{384,385}$ carbon nanotubes (CNTs), ${ }^{386}$ metal nanowires, ${ }^{387,388}$ conducting polymers, ${ }^{302,303}$ and MXenes $^{389}$ have been investigated as a substitute. However, the electrodes made up of these prevailing materials may suffer from limited transmittance in the bleached state.

(ii) Metal nanostructures. Metal grids and 1D noble metals nanoparticles such as $\mathrm{Ag}, \mathrm{Au}, \mathrm{Cu}$, and $\mathrm{Al}$ have been tested as coatings for wearable substrates, which exhibit decent flexibility and excellent conductivity (Fig. 27). ${ }^{390}$ The metal-based coated layer should retain a low refractive index in the visible region and d-band contribution to the plasma wavelength oscillations in the UV range $(\sim 320 \mathrm{~nm})$. The $\mathrm{d}-\mathrm{d}$ electronic transitions provide minimum reflectance of a shorter wavelength in the visible region closer to the plasma wavelength. ${ }^{300,391}$

Gold exhibits great potential because of its environmental stability and LSPR peaks that spectrally match with the solar emissions. ${ }^{293,393} \mathrm{Ag}$ nanowire-based films are rapidly emerging due to their excellent conductivity, transparency, softness, and toughness, making them an auspicious candidate for the electrodes of next-generation wearable optoelectronics. ${ }^{84}$ The refractive index of silver is 0.06 and its extinction coefficient of 3.59 at $\lambda=550 \mathrm{~nm}$ is the most ideal choice among the noble and non-noble metals in recent research. It has limited absorption in the visible region with a neutral color and has excellent optoelectronic properties. ${ }^{300,394,395} \mathrm{Cu}$ multi-layers films also increased in popularity and became the preferred choice for thin-coating technology because of their tunable performance and reduction in overall material size and cost. ${ }^{300}$ Metals nanowire-based electrodes have good connectivity, flexibility, and anti-deformation ability. However, they have certain limitations, where the nanowires have weak wire-to-wire interaction and poor adhesion with the substrates, making it easy for them to detach from the surface, thus shortening the coating lifetime. It is worth noting that pure metal nanowires are easily oxidized in air and have poor stability due to longterm electrochemical cycles. Therefore, new materials with ultrasmall size and excellent morphologies should be exploited to construct flexible substrates with lightweight, high electrochemical stability, high transparency, high interface adhesion of conductive layers, and high thermal management properties.

To improve the conductivity stability of silver-based transparent conductive electrodes, Cai's group ${ }^{83,114}$ and Li's group $^{299}$ proposed replacing ITO with silver grids as flexible transparent conductive substrates, as shown in Fig. 28. A high conducting silver grid/PEDOT:PSS hybridized composite film with outstanding stability was developed. An ultrathin layer of $\mathrm{WO}_{3}$ nanoparticle coating was formed on an Ag-grid/PEDOT:PSS hybridized film, exhibiting the performance of flexible/foldable electrochromics. The hybrid-blend structure showed a large optical modulation of $81.9 \%$ at $633 \mathrm{~nm}$, fast switching kinetics, and a high coloration efficiency of $124.5 \mathrm{~cm}^{2} \mathrm{C}^{-1}$. Moreover, the hybrid film showed outstanding electrochemical cycling stability (sustaining $79.1 \%$ of its initial 

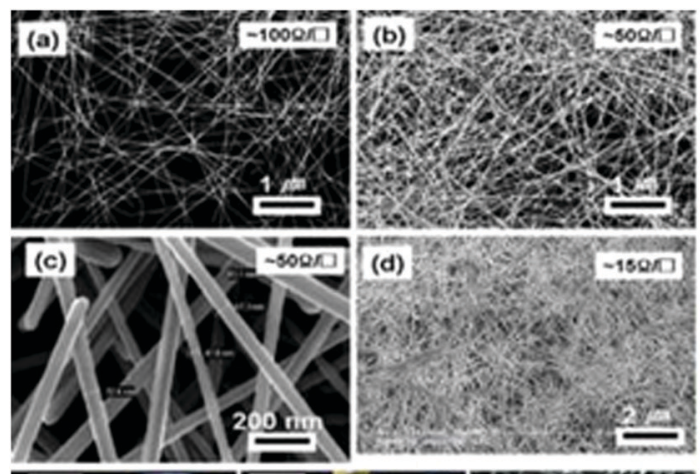

(d)
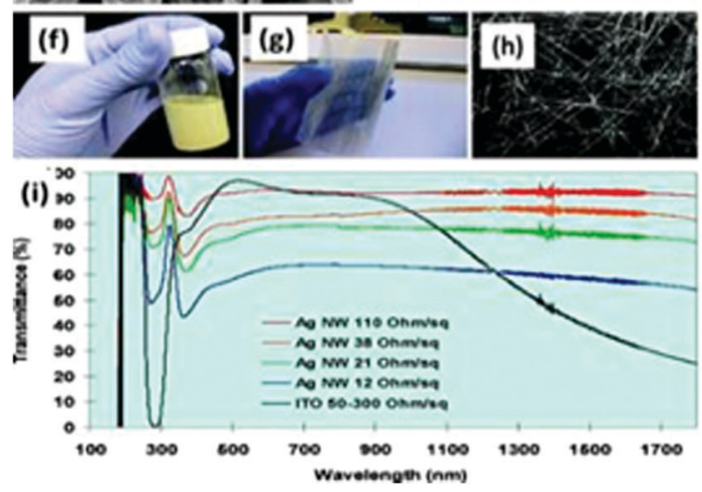
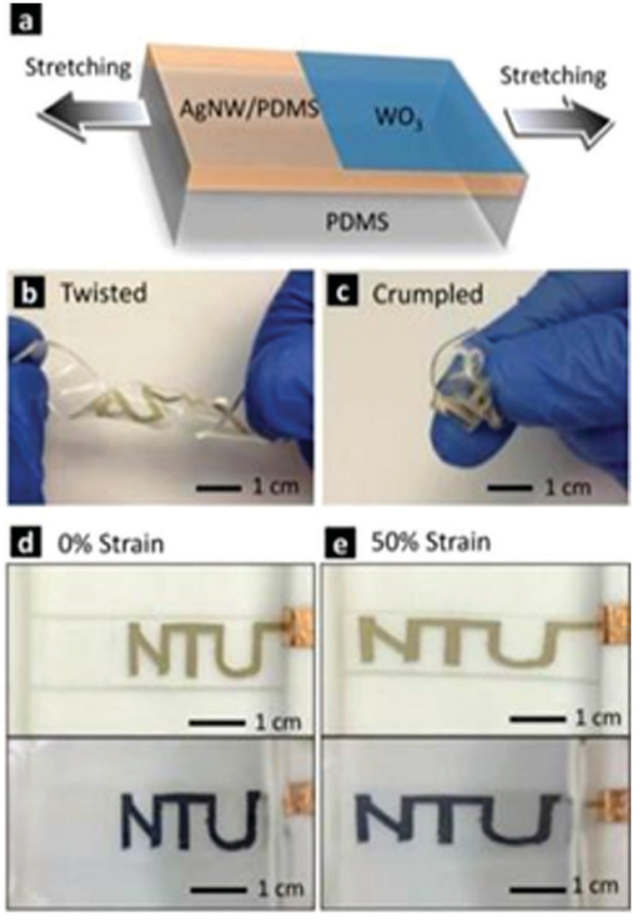

Fig. 27 (right) SEM images of Ag NW films produced from Ag NWs with a diameter of 40-100 nm and different densities lead to different sheet resistances of: (a) 100, (b and c) 50, and (d) $15 \Omega \square^{-1}$. (f) $2.7 \mathrm{mg} \mathrm{mL}^{-1} \mathrm{Ag}$ NW ink in ethanol solvent. (h) SEM image of Ag NW coating shown in panel c. The sheet resistance is $\sim 50 \Omega \square^{-1}$. (i) Optical transmittance of transparent Ag NW electrodes measured with a UV-vis spectrometer, without including the substrates. Reproduced with permission. ${ }^{387}$ Copyright $\mathbb{C}$ 2010, ACS. (Left) (a) Schematic of the stretchable EC device. (b and c) Stretchable device is twisted and crumpled, showing excellent mechanical robustness. ( $d$ and e) Examples of the patterned device in the bleached and colored states at 0 and $50 \%$ strain, respectively. Reproduced with permission. ${ }^{392}$ Copyright $\mathbb{C} 2014$, ACS.

transmittance modulation after 1000 cycles) and remarkable mechanical flexibility (optical modulation decay of only $7.5 \%$ after 1200 compressive bending cycles). Consequently, a smart supercapacitor was developed as a conventional energy-storage device, which can simultaneously monitor the energy storage levels through rapid and reversible colour changes, even at high charge and discharge processes. The high-performance Ag-grid/ PEDOT:PSS hybridized transparent films demonstrate a promising topology and a wide range of developing flexible electronics and photoelectronics. The high angle flexibility and bending nature of $\mathrm{Ag}$ nanowire-coated substrates maintain interconnectivity and can still illuminate light-emitting-diodes (LEDs) and bulbs after severe mechanical bending and deformation (Table 3). ${ }^{37}$

(iii) Carbon nanotubes (CNTs). Due to their distinctive 1D nanostructure and unique mechanical and electrical properties, CNTs have achieved potential in many types of electronic devices. In particular, their inherent high conductivity, excellent flexibility, and stability, and relatively easy and low-cost preparation make them a promising electrode candidate for next-generation smart electronics. When CNTs act as electrodes for EC devices, they can also be employed as promising sensing elements and stretchable/flexible electronics for the prospective development of soft and versatile ends. Moreover, because of their compatible functionalized structure, they can also function as both an electrode candidate and EC smart material, which will further shorten the structure of EC devices and reduce their manufacturing costs. ${ }^{84}$

(iv) Graphene. The thinnest 2D carbon material has wide application prospects in many research fields because of its excellent electronic, optical, mechanical, and thermal properties. ${ }^{381}$ Graphene electrodes made of mechanically cracked graphene, CVD-grown graphene, or mass-produced graphene derivatives from bulk graphite have been used in a wide range of applications as an alternative to ITO transparent electrodes such as light emitting-diodes, touch screens, solar cells, field-effect transistors, batteries, supercapacitors, and sensors. $^{381}$

(v) MXenes. Recently 2D transition metal carbides, nitrides, and carbonitrides known as MXenes have achieved wide research interest due to their flexibility, superior mechanical strength, physical/chemical properties, and multiplex tremendous functionalities. ${ }^{389}$ Because of their excellent electrochemical stabilities, mechanical properties, high ion adsorption capacities and charge transfer, metallic conductivities, and unique topology, MXenes have demonstrated innumerable potential applications in structural composites, electromagnetic interference (EMI) shielding materials, lithium-ion batteries (LIBs), supercapacitors (SCs), electrocatalysts, electronics, and topological insulators. ${ }^{256,389,412}$ Interestingly, 2D 

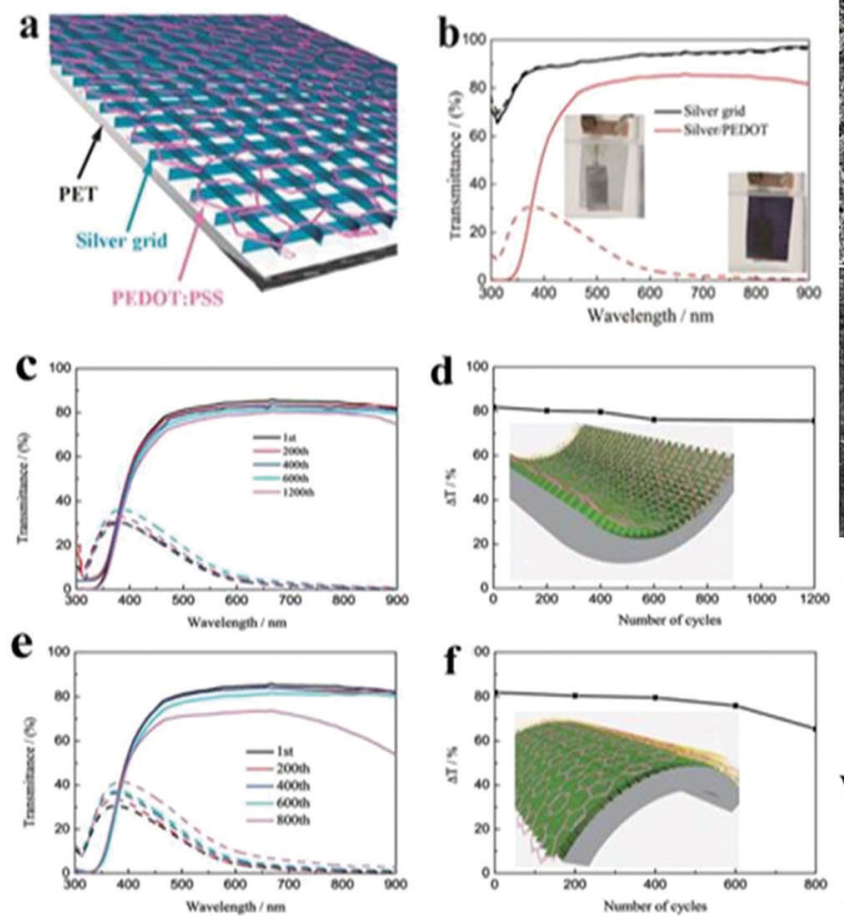
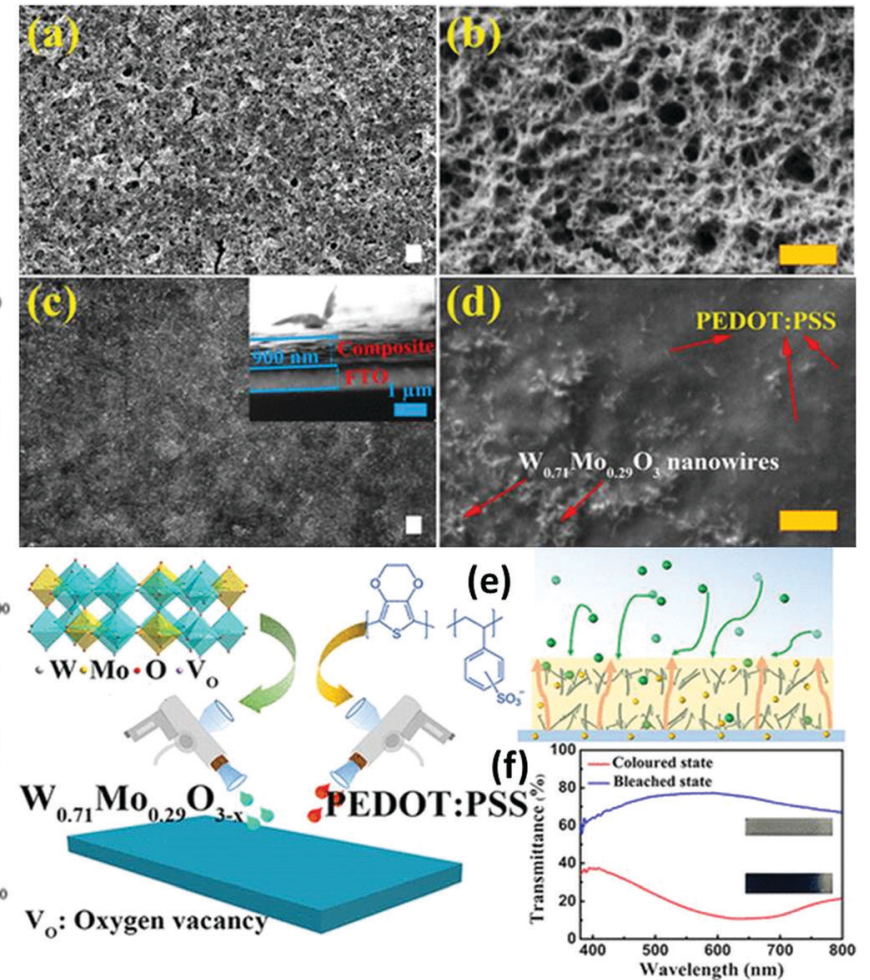

Fig. 28 Left (a) Schematic illustration of the structure of the silver grid/PEDOT:PSS hybrid film. (b) Transmittance spectra of WO 3 in the colored ( $-0.7 \mathrm{~V}$ ) and bleached $(0.1 \mathrm{~V})$ state over the wavelength range of $300-900 \mathrm{~nm}$. (c-f) Transmittance and optical modulation changes of WO $\mathrm{O}_{3}$ on the silver grid/ PEDOT:PSS hybrid film in the bleached and colored state under repeated compressive bending (c and d) or tensile bending (e and f) with a curvature

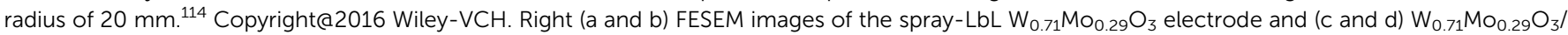
PEDOT:PSS electrodes. (e) Schematic illustration and (f) optical transmittance. Reproduced with permission. ${ }^{299}$ Copyright $(2018$ ACS.

Table 3 Transparent conductive substrates for EC devices

\begin{tabular}{|c|c|c|c|}
\hline Electrode materials & Deposition method & Properties & Ref. \\
\hline FTO thin film & Spray pyrolysis & $R=\sim 7 \times 10^{-4} \Omega \mathrm{cm} ; T=\sim 90 \%$ & 397 \\
\hline $\mathrm{Ag}$ NWs/PI & Wet process & $R=25 \Omega \mathrm{sq}^{-1}, T=86 \%$ & 398 \\
\hline Ag NWs/PET & Lithographic filtration & - & 392 \\
\hline Ag NWs/glass & Spin coating & $R=10 \Omega \mathrm{sq}^{-1}, T=60 \%$ & 85 \\
\hline Ag NWs/PDMS & Spray coating & $R=20 \Omega \mathrm{sq}^{-1}, T=85 \%$ & 400 \\
\hline CNT/PDA nanofibers & Spinning coating & $R=100-1000 \mathrm{~S} \mathrm{~cm}^{-1}$ & 403 \\
\hline CNT/PDMS & Paving method & $R=0.1-1 \mathrm{k} \mathrm{S} \mathrm{cm}^{-1}, T=80 \%$ & 404 \\
\hline Graphene/PET & Layer formation & $R=1 \sim 2 \mathrm{k} \Omega \mathrm{sq}^{-1}, T=97 \%$ & 405 \\
\hline Graphene/PET-ITO & Electrodeposition & $R=-, T=80 \%$ & 406 \\
\hline $\mathrm{N}$-Graphene/glass & LPCVD & $R=1.1 \mathrm{k} \Omega \mathrm{sq}^{-1}, \mathrm{~T}=93 \%$ & 407 \\
\hline RGO/Cu NWs/glass & Spin coating/spray process & $R=34 \pm 2.6 \Omega \mathrm{sq}^{-1}, T=80 \%$ & 408 \\
\hline Ag NWs/PEDOT:PSS/PET & Spray process & $R=10 \Omega \mathrm{sq}^{-1}, T=86 \%$ & 409 \\
\hline
\end{tabular}

MXene nanosheets and their derivatives can be produced via wet-chemical synthesis, which makes it easy to self-assemble hybrid-films and unique structures. ${ }^{256,412,413}$ Thus, the effective combination of the mechanical and functional properties of MXenes emerges as a promising opportunity to design nanoscale flexible, foldable, and wearable EC devices on a large scale with superb durability.
6.2.2. Ion conducting electrolytes. An electrolyte is the conducting ion reservoir between the EC materials and electrodes. It can exist as a liquid, gel, polymer, or in the solid state, either as solid thin films or a polymer layer. ${ }^{104,414,415}$ Electrolytes play a crucial role in the electrochemical reactions, providing a path for ions between the electrodes and avoiding the flow of electrons due to the cation-host interaction. ${ }^{104,416}$ 
The ion transfer speed and efficiency have a great impact on the switching performance, including response speed, coloration efficiency, and cyclic stability. Granqvist well explained the corresponding types of glass-based rigid electrolytes and polymer-based flexible electrolytes. ${ }^{417}$ Lee's team reviewed solid-state and polymer-based flexible electrolytes, ${ }^{418-420}$ whereas, Thakur et al. ${ }^{104}$ reviewed the development of polymer electrolytes, and Li et al. ${ }^{380}$ also reviewed the solid-state gelelectrolytes for flexible/stretchable devices. Similarly, Yun et al. ${ }^{421}$ also demonstrated non-volatile, Li ion-based gel electrolytes for $\mathrm{WO}_{3}$-based flexible EC devices. In practical applications, solid-state electrolytes, for example polymers, ions, gels, and ion-conducting ceramic-based electrolytes are required to avoid certain limitations ${ }^{419}$ caused by the use of liquid electrolytes, such as leaks, fire, and even deformation/breaking of the glass, especially in large-area EC window applications. Monovalent cationic-electrolytes are used in $\mathrm{WO}_{3}$-based EC applications, in which tiny size ions can easily enter/exit the hostlattice. However, they have some disadvantages. The cyclic stability of the EC devices composed of amorphous $\mathrm{WO}_{3}$ films is generally lowered in acidic electrolytes. This is because of the corrosion effect of $\mathrm{H}$-ions in acidic media. Due to the low electrode proficiency, the production of $\mathrm{H}_{2}$ bubbles has also been observed on the surface of electrodes. The best choice is lithium-salt-based electrolytes, which can be used alternatively to achieve tremendous EC stability and prolonged switching kinetics. Polymer electrolytes have been employed for advanced electrochemical and EC devices. ${ }^{415,420,422-426}$ However, it is necessary to develop and select an electrolyte with an ideal performance for EC smart windows.

6.2.3. Ion storage/conductive oxide counter electrodes (anode). Complementary electrodes in EC devices play a key role in balancing the charge and ion storage properties. The electrode with ion-storage properties is generally referred to as the counter electrode, where the electrode with the EC layer in smart devices is known as the working electrode. When an appropriate electric current is applied, the EC materials on the working electrode are oxidized/reduced, and similarly, the equivalent reduction/oxidation of the electroactive materials occurs simultaneously on the counter electrode (Fig. 1). Nextgeneration smart windows generally require a counter electrode with (i) high transmittance, (ii) outstanding charge capacity, and (iii) low interface resistance, matching the working electrode. $\mathrm{NiO}, \mathrm{IrO}_{2}, \mathrm{CeO}_{2}$, and $\mathrm{V}_{2} \mathrm{O}_{5}$ can be used as anodically chromic and/or charge storage materials, which are termed the counter electrode. The conventional EC windows show colour modulation in a complementary fashion to the primary material. Among them, $\mathrm{NiO}$ is the most popular, which shows a grey colour in the oxidized state. In NiO, the EC coloration is due to the surfaces and grain interfaces by the conversion of hydrated $\mathrm{Ni}$ oxide into hydroxide and then oxy-hydrate phases. The overall electrochromism occurs under the Bode-reaction scheme $^{427}$ between Ni-hydroxide and oxy-hydroxide, as explained earlier.

Choi et al. ${ }^{428}$ deposited different size NiO particles on ITO glass through dry deposition, as shown in Fig. 29a-c. The electrochemical results showed that the nanoporous NiO film had a high charge storage capacity $\left(49.49 \mathrm{mC} \mathrm{cm}^{-2}\right)$ and lower charge transfer resistance $(0.49 \Omega)$. The porous structure and large active surface area enabled better contact with the electrolyte and ion diffusion in the nanoscale NiO fabricated film. The film showed an excellent transmittance modulation (42\%), with a stable switching speed and electrochemical cycles. Kim et al. ${ }^{429}$ fabricated N-doped carbon-based porous NiO films via a sol-gel process with the assistance of oleylamine and amide condensation. The film showed the coloration efficiency of $48.5 \mathrm{~cm}^{2} \mathrm{C}^{-1}$, color/bleach speed of $3.2 / 2.7 \mathrm{~s}$, and specific capacitance of $235.8 \mathrm{~F} \mathrm{~g}^{-1}$ with the applied current density of $2 \mathrm{~A} \mathrm{~g}^{-1}$ (Fig. 29d-h). Wang et al. ${ }^{430}$ reported the electrochemical deposition of $\mathrm{H}_{3} \mathrm{PW}_{12} \mathrm{O}_{40}\left(\mathrm{PW}_{12}\right)$ on a $\mathrm{TiO}_{2}$ nanoparticle film coated on FTO glass, which functioned as an electron acceptor and redox shuttle for charge balancing of the $\mathrm{TiO}_{2}$ nanoparticles as the counter electrode (Fig. 29i-l). The hybrid film achieved $68 \%$ optical modulation at $1.5 \mathrm{~V}$ to $-2.0 \mathrm{~V}$ applied voltage, cyclic stability (2500 cycles), and good coloration contrast.

6.2.4. EC material cathode $\left(\mathbf{W O}_{2.72}\right)$. The EC layer is the main component in smart windows technology (Fig. 1), which consists of EC materials (Sections 2 and 3). The reversible electrochemical redoxation occurs with the insertion of ions through an applied electric potential and EC materials change their optical absorption properties dynamically. Among the EC materials, nonstoichiometric $\mathrm{WO}_{3-x}$ has received wide research interest due to its unique properties, as has been explained in detail in Sections 2-5.

\section{Challenges and perspective outlooks}

\subsection{Challenges}

Chromogenic materials have been used for dynamic switchable smart windows; however, the commercialization of EC windows in the market is still hindered because of major problems including high manufacturing costs, material implementation, durability, and reliability. The significant challenges faced in the synthesis of $\mathrm{WO}_{3-x} / \mathrm{WO}_{3}$ nanostructures on a large scale and efficient film preparation are as follows:

(i) Lack of standard synthetic methods for ultra-small size $\mathrm{WO}_{2.72} / \mathrm{WO}_{3}$ particles with a high surface area and EC electrode fabrication. It is very difficult to evaluate/compare the EC performance (coloration efficiency, switching speed, cycling life, cell stability, durability, etc.) reported in the literature.

(ii) Considering industrial applications, the synthesis methods are still far from market penetration due to the technical aspects and factors including quality, safety, cost, environmental hazard, mass production, and processability.

(iii) The phase-pure growth of $\mathrm{WO}_{2.72}$-based materials on the surface of electrodes with a controllable morphology and dimensions can achieve excellent EC performances due to fast electron/ion transportation, but this has been largely ignored. In the meantime, the loading (density/\%) of $\mathrm{WO}_{2.72}$-based 


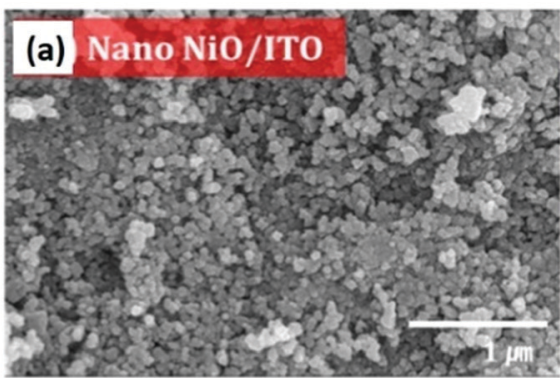

(d)

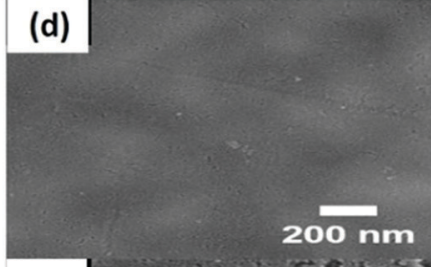

(f)

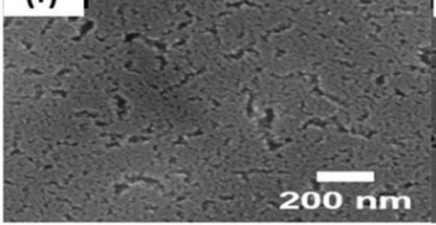

(i)

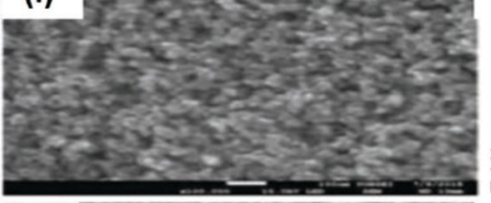

(j)

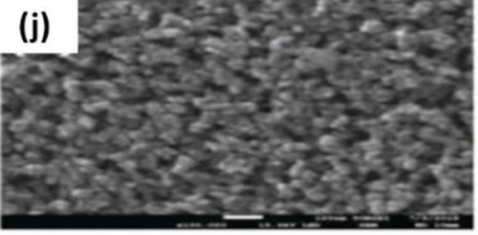

(e)

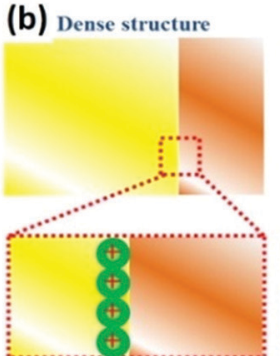

\section{.}
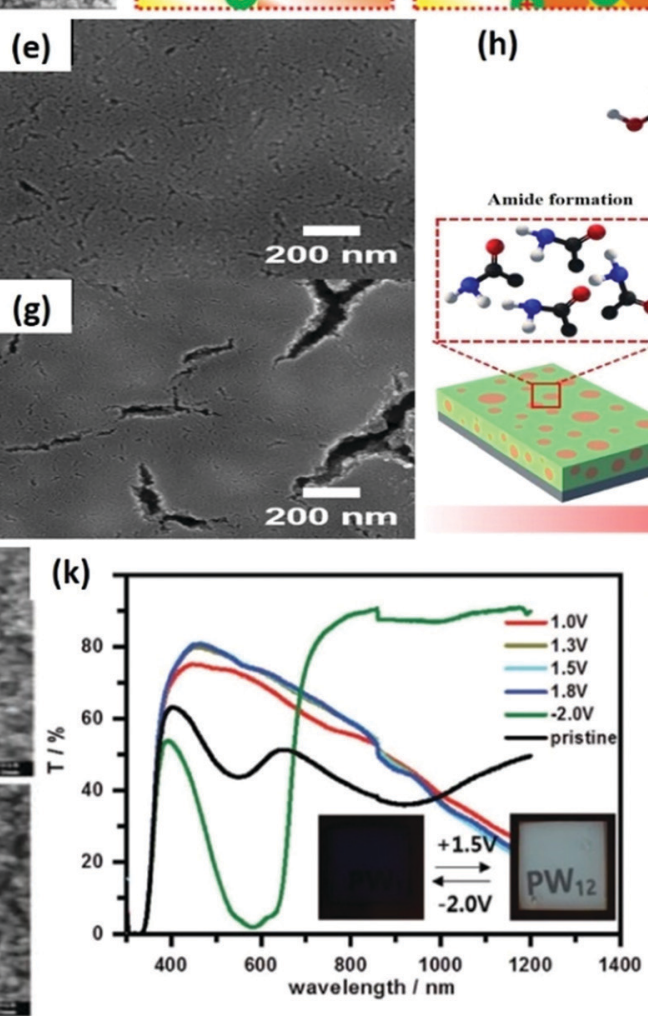

(h)
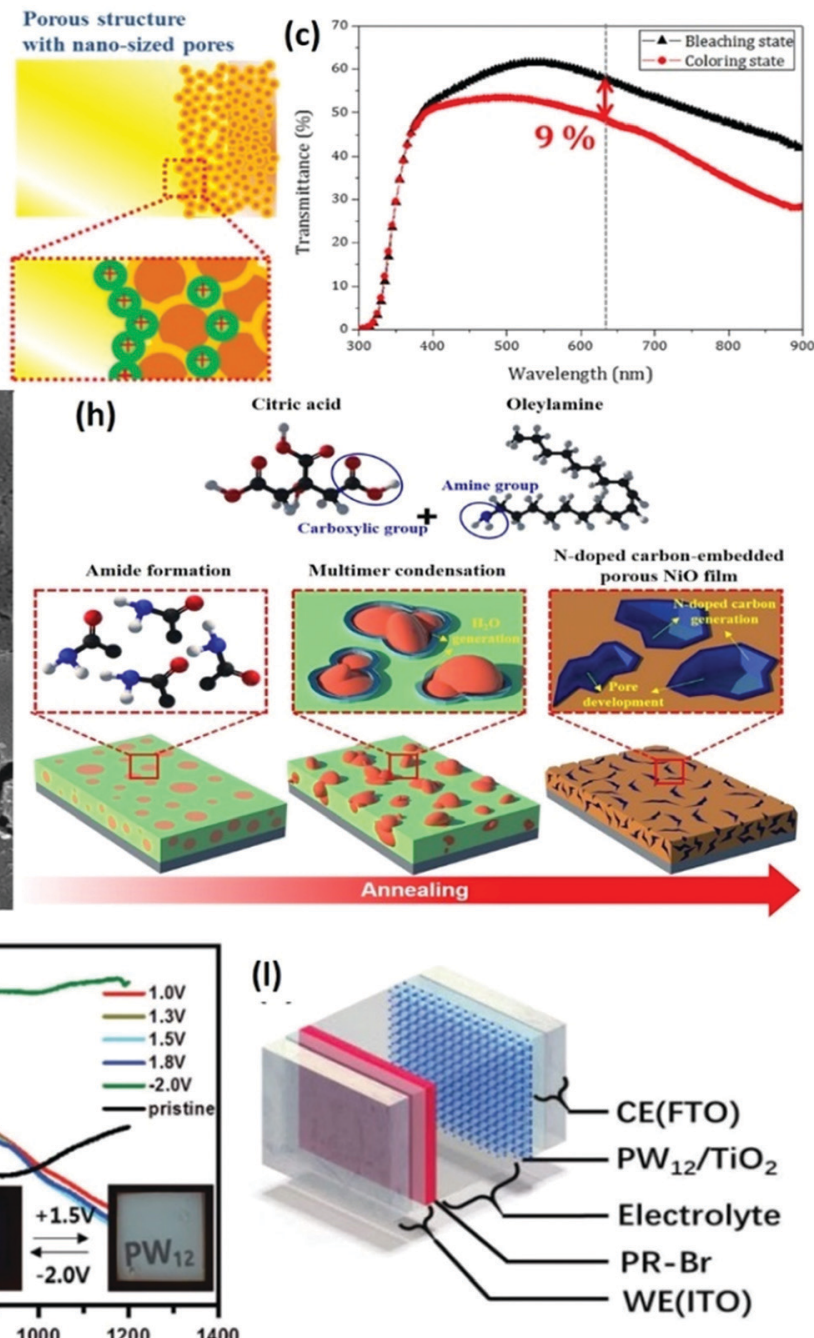

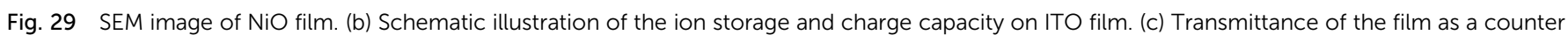

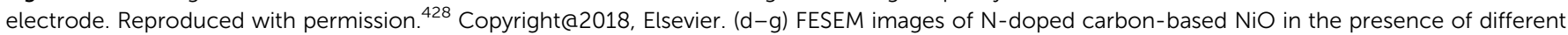

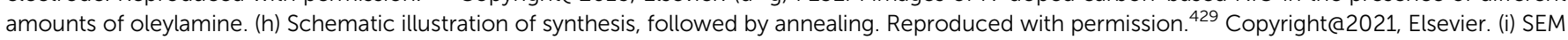

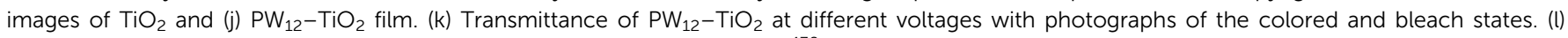
Schematic of the various layers in the device. Reproduced with permission. ${ }^{430}$ Copyright@2019 WILEY-VCH.

materials, electrode area, EC performance parameters, and other conditions should be stipulated.

(iv) The active $\mathrm{WO}_{2.72}$ nanostructure, plasmonic-doped or plasmonic-nanocomposite powders are considered ideal for practical applications due to the presence of conductive agents. However, smart techniques are required to overcome the inherent disadvantages and phase purity of each component.

(v) For generating multifunctionality, the shape, size and tunable porous film coatings of $\mathrm{WO}_{3-x}$-based plasmonic composites on wearable substrates acquire lightweight, high active surface area, and excellent conductivity. However, the charge/ discharge capacity and expenses utilizing stretchable/deformable substrates are a key challenge for global researchers. Similarly, the optical modulation in white light and photothermal instability also need to be optimized. (vi) When $\mathrm{WO}_{3} / \mathrm{WO}_{3-x}$ tailored structures are deposited on a conductive substrate support, they can achieve relatively high electrochemical efficacy. However, at the device level, the cost may be quite high and the performance poor, with failure to reach the optical saturation threshold limit. Moreover, fast redox reactions/large electrochemical cycles result in EC dysfunction, and thus yellowing appears.

(vii) The mechanism through which oxygen vacancies/crystal defects and the electron/hole coupling in sub-stoichiometric $\mathrm{WO}_{3-x}$ affect EC and electrochemical properties is not well understood experimentally and/or theoretically.

(viii) Self-chargeable, foldable/flexible smart windows with unique structural designs, non-sacrificing optical properties, and cost-effective materials are a growing future demand and can be implemented first in unique wearable applications, as part of energy storage systems. 


\subsection{Outlook}

7.2.1. Energy savings and multifunctional features. Due to their integration with multifunctional features such as energy storage, energy harvesting, and environmental sensors, the next-generation EC smart windows will be promising for novel diverse applications. ${ }^{33,34,40,66,103,196,372}$ Tungsten oxide-based coated films achieve dark blue color with an applied voltage and also become absorbing and store electric current. In selfpowered configurations, solar energy is absorbed and converted to electrical energy. The stored energy can be used to power EC windows to initiate the redox reaction which dynamically changes the color of the windows, thus effectively regulating the solar loads in buildings.

Due to their similarities with energy storage devices, e.g., supercapacitors and batteries, EC smart windows are known as transparent electrodes with reversible color states. Electrochromic supercapacitor devices (ESDs) with multicolor changes, energy harvesting and energy storage capacity, cyclic stability, and the realization of the quantitative monitoring of energy storage level of an ESD are very important to realize the practical application of the device. ${ }^{431,432}$ Conducting polymers have been studied as electrode materials for ESDs due to their rich color change and good energy storage performance. ${ }^{431}$ Pseudocapacitive materials simultaneously enable a high rate, high energy density, and energy storage systems based on ion intercalation. ${ }^{433,434}$ They can be incorporated into electrodes to minimize the mass-transfer limitations due to thickness effects, and electrolyte starvation/low particle-to-particle electron transport. For this purpose, $\mathrm{WO}_{3-x}, \mathrm{NbO}_{x}, \mathrm{MoO}_{2}$, and $\mathrm{TiO}_{2}$ with $\mathrm{Mn}, \mathrm{Ni}$, and Co oxides are appealing because of their properties related to storing electric charges and dynamic modulation of LSPR-absorption through capacitive charging/ discharging. ${ }^{347}$ The 2D-layered materials known as MXenes have attracted much attention for applications in energy storage, optoelectronics, and photocatalysis. ${ }^{372}$ MXenes demonstrate excellent flexibility, are easy to process, with an ultrafine thickness of high conductivity, and possess the highest electromagnetic interference shielding effect. Thus, self-assembled MXene- $\mathrm{WO}_{3-x}$ heterostructures should be employed as flexible and foldable energy storage EC films. The MXenes as a flexible transparent electrode and $\mathrm{WO}_{3-x}$ as the flexible EC layer are expected to achieve excellent performances in flexible EC windows on a large scale. The charge-storage mechanism should be better understood based on pseudocapacitive/ battery-type materials linked with $\mathrm{WO}_{2.72}$ to enrich the capacitive behavior for achieving self-powered multifunctionality. In addition, there is no clear quantitative relationship between the color change parameters and the energy storage level to achieve quantitative monitoring. This is because the constructed ESD is used to estimate the energy storage state through a color change. Under different charge-discharge voltages, the ESD displays different color.

Besides solar loads, human motions, and daily activities such as talking, walking, and running can produce a large amount of mechanical energy. Sensors that can harvest energy produced by human activities have been developed.
Self-powered Wi-Fi-connected smart windows integrated with environmental sensors can read room occupancy and detect changes in the weather, gases, light, temperature, and mechanical deformations. These all-in-one self-driven, shape-adaptive versatile smart windows can be self-chargeable with solar energy/heat and gather energy by human interaction/motions, and various other types of sustainable mechanical energy conversion resources would be promising improvements.

7.2.2. Reducing toxicity. LSPR nanocrystals anchored/doped with $\mathrm{WO}_{3-x}$ for the optical transmission of electrochromicphotothermal conversion of visible and NIR-light can effectively reduce the risk of microorganisms and are expected to be used as sterile smart windows. Au, Ag, Rh, Pd, and Pt LSPR have been widely used for cancer therapy. Thus, the broadband nonradiative plasmon decay of LSPR nanocrystals is assumed to be highly tunable as an electric field on the surface of $\mathrm{WO}_{3-x}$. Surface-decorated $\mathrm{WO}_{3-x}$ plasmonic composites are very useful for reducing the thermal damage threshold due to electronphonon and phonon-phonon coupling. MXenes are also promising antibacterial agents, with higher efficiency than graphene in diminishing bacterial cell viability. ${ }^{372}$ Thus, Au-decorated/selfassembled $\mathrm{Au}-\mathrm{MXene}-\mathrm{WO}_{3-x}$ heterostructure-based films exhibit promising transparency, flexibility, EC-photothermal sterility, and effective modulation of visible and NIR light. The photothermal transformation will be attained in the colored state, which is ascribed to the coupling of traditional visible-band optical switching with the NIR-LSPR extinction, and thus can act as high-tech sterile EC smart windows. These multifunctional sterile EC smart windows will be particularly useful in buildings, hospitals, highlatitude zones, and aircraft.

7.2.3. Coloration enhancement/poly-electrochromism. Due to electron/ion intercalation, tungsten oxide films achieve dynamic controllable blue color, which has a great influence on light absorptivity. The color preferences are closely associated with the cultural background, where the deep blue color is very suitable in China and Pakistan. The color development choices may vary from country to country. Doping can pave a path to change the colors by shifting the adsorption edges. Thus, doping and co-/multi-doping must be tested at the atomic level through experiments and simulation studies, together with further structural design at the nano-level. Hybridization with either inorganic/organic candidates is an efficient way to modulate colors/multicolor; however, the complicated fabrication strategies should be exploited further with more creative ideas.

In recent years, DMD (dielectric/metal/dielectric) blends have attracted increasing attention, in which the $\mathrm{WO}_{3}$ membrane can play a dual role of reflecting light energy in the bleaching state and absorbing in the colored state. In addition, $\mathrm{Ag}$ is an excellent heat-regulating material, which can also replace expensive substrates, e.g., FTO and ITO. The presence of extreme transmissive states in $\mathrm{WO}_{3-x} / \mathrm{Ag} / \mathrm{WO}_{3-x}$ allows the combination of numerous EC materials (hybrids) with controlled redox reactions to produce a variety of colors and bleach states with color coordination. In addition, the coloration efficiency and color coordination for $\mathrm{WO}_{2.72}$ electrochromism have not been investigated systematically, which 
complicates further advanced research. In the DMD architecture, the $\mathrm{Cu}$ and/or $\mathrm{Al}$ metals sandwiched between EC material layers are reliable and thermally reflective. Moreover, they can be promising in terms of cost, durability, and ease of processing. Researchers found that $\mathrm{WO}_{3}, \mathrm{TiO}_{2}, \mathrm{SnO}_{2}, \mathrm{ZnO}, \mathrm{CuS}, \mathrm{ZnS}$, AlN, $\mathrm{MoO}_{2}$, and $\mathrm{Nb}_{2} \mathrm{O}_{5}$ can form functional multilayer structures that can functionalize conductive metals, for multicoloration in EC smart windows.

7.2.4. Reversibility, durability, and inadequate switching. It is worth mentioning that $\mathrm{WO}_{2.72}$ films suffer from degradation due to prolonged switching (ion-intercalation/deintercalation), thus affecting the optical modulation and cyclic stability, and consequently the device performance. Continuous currentdriven ion de-trapping and self-healing are a successful way to eliminate the ion-trapping resulting in degradation and reduction in EC properties. Therefore, the film can be refreshed and attain its initial state, which is a successful effort to design super EC substrates for smart windows and other powered optoelectronics. Self-healing EC materials (e.g., viologens/polymers) can perform in situ redox reactions with excessive $\mathrm{Li}^{+}$ions to reduce resistance transfer and avoid interface obstacles. The reversible Diels-Alder cross-linking network ${ }^{435}$ structure can heal the cracks and improve the reliability of the $\mathrm{WO}_{3-x} \mathrm{EC}$ layer. The formation of an inert oxide shell separating $\mathrm{WO}_{3-x}$ from oxygen also induces a characteristic effect, but the controllable formation of the core-shell nanoarchitecture is not very easy. Other essential variables are the $\mathrm{WO}_{2.72}$ response time under various film thicknesses, material loading per unit area, and long-term environmental stability. For additional protection and to avoid unnecessary physical/chemical reactions or environmental strain (damage, ultraviolet, heat, water vapor, and/or oxygen), smart window devices can be well sealed by covering curtains, as shown in Fig. 1, which helps improve their stability, performance, and reliability.

7.2.5. Dual/multifunction. Self-powered EC windows can offer numerous ways to reconfigure sustainable energy resources in smart windows, thus reducing energy consumption by minimizing the heating loads, cooling loads, lightings, and also protecting the privacy of users. Smart windows can also store energy during the electrochemical process through a rational circuit in the same electrolyte. Consequently, the stored energy can be utilized to derive other functional devices. Tremendous progress has been made in smart electronics and dual/multifunctional windows for practical applications, in which the $\mathrm{WO}_{3}$ and $\mathrm{NiO}^{436}$-based EC supercapacitors have been widely demonstrated. ${ }^{437}$ Dual-function EC supercapacitors serve as an energy storage device and electrical power source simultaneously, displaying real-time capacity in the color state. ${ }^{431,432}$ The unique characteristics achieved include coloration/bleaching and charging/discharging, large transmittance contrast $(\sim 91 \%)$, high coloration efficiency $\left(\sim 61.9 \mathrm{~cm}^{2} \mathrm{C}^{-1}\right)$, high areal capacitance $\left(\sim 13.6 \mathrm{mF} \mathrm{cm}^{-2}\right)$, and good charging/ discharging cyclic stability. ${ }^{437}$ A low doping amount of less than $10 \mathrm{~mol} \%$ can significantly enhance the EC performance. ${ }^{436}$

Moreover, smart windows with unique novel multifunctional features extend their utility. Metal oxides become opaque upon ion extraction such as oxides of nickel and iridium or PEDOT/PSS materials. Among them, Ni is more functional, relatively common, and less expensive, which make it preferable for large-scale production. A device that can combine two types of EC functional materials, e.g., $\mathrm{WO}_{3-x}$ and $\mathrm{NiO}$, will be more advantageous, where the charges transfer from $\mathrm{Ni}$ to $\mathrm{W}$ oxide. In the opposite direction, the EC materials will be bleached (Fig. 1). It should be stated that the EC properties of $\mathrm{NiO}$ are different from that of $\mathrm{WO}_{3}$ in the case of $\mathrm{Li}^{+}$ion-based electrolytes. The reason for this is that NiO involves surface sites for the exchange of both cations and anions. ${ }^{438}$ However, these novel multifunctional EC smart windows should be designed to enrich various EC materials coatings to convert solar loads into useful energy simultaneously, ${ }^{34}$ or to allow the transmission of visible light but reflect NIR light. Hybrid functional compatible electrochromics in a single platform are significant alternatives in balancing the performance limitations of one type of EC material by supplementing with the required activity of the competent material. Thus, enriching versatility in a smart way and minimizing the overall device size with superb service life will be fundamental approaches in the future.

7.2.6. Advancements in $\mathbf{W O}_{2.72}$ nanoparticles and film formation strategies. $\mathrm{W}_{18} \mathrm{O}_{49}$ nanoparticles should be considered for commercialization by controlling their dimensions, size, dispersibility, phase purity, synthesis routes, and also coating strategies, such as annealing the coated film, surface porosity and adhesion of films with substrates, nature of the conductive substrates, nature of electrolytes, etc., and quantity/ quality regulation of all components. Plasmonic metal-doped $\mathrm{WO}_{3-x}$ and its composites in the desired shape, size, and tunable porous film coatings with a high active surface area, excellent conductivity, and lightweight on stretchable/deformable substrates should be the focus for scaling up. PED, PLD, and HiPIMS can be effective deposition methods for the coating of $\mathrm{WO}_{2.72}$-based EC materials. ${ }^{37,86,114,309,310,392}$ The wetdeposition techniques require the preparation of suitable inks from nanostructured EC materials, and thus the ink preparation can be the main components in assessing the coating strategies. Inkjet printing is a cost-effective and highly attractive industrial technology that can be used to manufacture EC materials on a variety of substrates. Moreover, it allows the coating of EC materials in a precise location with a controllable thickness.

The traditional vacuum evaporation, magnetron sputtering, and spin-coating methods are not suitable to achieve large-area coatings, controllable thickness, and low cost. Recently, our team has focused on different ways to produce ultra-thin films by rolling, spraying, casting/screen printing, and spin coating on various substrates, which shall remarkably reduce costs and readily achieve high-quality EC/TC smart responsive coatings to fulfill the huge market demands. However, the performance optimization and the commercial production still need more effort, as the researchers require cost-effectiveness, manufacturability, and market demand user willingness and policies. Thus, suitable and sustainable solutions to the problems 
addressed in this review regarding EC smart windows need to be found in the future.

\section{Conflicts of interest}

There are no conflicts to declare.

\section{Acknowledgements}

This work was supported by the National Natural Science Foundation of China (21876062), and the Key R\&D Program of Shandong Province (2019GSF109071). Thanks to the China Scholarship Council (2017DFH011650) for International Doctoral Students in China. This work was also fully sponsored by Shandong Shenna Smart Advanced Materials Co. Ltd.

\section{References}

1 C. G. Granqvist, in Handbook of Inorganic Electrochromic Materials, ed. C. G. Granqvist, Elsevier Science B.V., Amsterdam, 1995, pp. vii-viii, DOI: 10.1016/B978044489930-9/50000-3.

2 A. Pawlicka, C. O. Avellaneda, M. M. Silva and F. Kajzar, Nonlinear Opt., Quantum Opt., 2019, 50, 165-182.

3 S. K. Deb, Philos. Mag., 1973, 27, 801-822.

4 S. K. Deb, Appl. Opt., 1969, 8, 192-195.

5 C. Granqvist, Thin Solid Films, 2014, 564, 1-38.

6 J. S. E. M. Svensson and C. G. Granqvist, Sol. Energy Mater., 1984, 11, 29-34.

7 C. M. Lampert, Electrochromic materials and devices for energy-efficient windows, Lawrence Berkeley Lab., 1984.

8 Y. Ke, J. Chen, G. Lin, S. Wang, Y. Zhou, J. Yin, P. S. Lee and Y. Long, Adv. Energy Mater., 2019, 9, 1902066.

9 H. Khandelwal, A. P. H. J. Schenning and M. G. Debije, Adv. Energy Mater., 2017, 7, 1602209.

10 H.-N. Kim and S. Yang, Adv. Funct. Mater., 2020, 30, 1902597.

11 C. Bechinger, S. Ferrere, A. Zaban, J. Sprague and B. A. Gregg, Nature, 1996, 383, 608-610.

12 S. Wang, W. Fan, Z. Liu, A. Yu and X. Jiang, J. Mater. Chem. C, 2018, 6, 191-212.

13 Y. Wang, E. L. Runnerstrom and D. J. Milliron, Annu. Rev. Chem. Biomol. Eng., 2016, 7, 283-304.

14 A. Cannavale, P. Cossari, G. E. Eperon, S. Colella, F. Fiorito, G. Gigli, H. J. Snaith and A. Listorti, Energy Environ. Sci., 2016, 9, 2682-2719.

15 Y. Ke, C. Zhou, Y. Zhou, S. Wang, S. H. Chan and Y. Long, Adv. Funct. Mater., 2018, 28, 1800113.

16 L. Peng, B. Su, A. Yu and X. Jiang, Cellulose, 2019, 26, 6415-6448.

17 X. Jiang, M. Soltani, E. Haddad, R. Kruzelecky, D. Nikanpour and M. Chaker, J. Spacecr. Rockets, 2006, 43, 497-500.

18 L. Peng, W. Fan, D. Li, S. Wang, Z. Liu, A. Yu and X. Jiang, Adv. Mater. Technol., 2020, 5, 1900599.
19 L. Liu, M. Liu, W. Fan, C. Zhao, X. Jiang, A. Yu and J. Wu, CN Pat., 109575713A, 2019.

20 M. Liu, B. Su, Y. V. Kaneti, Y. Tang, X. Jiang, A. Yu, Z. Chen, Y. Gao, Y. Yuan and L. Jiang, ACS Nano, 2017, 11, 407-415.

21 H. Zhu, Z. Zhang, M. Liu, W. Fan and X. Jiang, J. Nanosci. Nanotechnol., 2019, 19, 3597-3603.

22 H. Xu, Z. Dai, C. Wang, K. Xu, F. Ma and P. K. Chu, J. Mater. Chem. C, 2018, 6, 7896-7904.

23 S. Wang, M. Liu, L. Kong, Y. Long, X. Jiang and A. Yu, Prog. Mater. Sci., 2016, 81, 1-54.

24 P. Castillero, V. Rico-Gavira, C. López-Santos, A. Barranco, V. Pérez-Dieste, C. Escudero, J. P. Espinós and A. R. González-Elipe, J. Phys. Chem. C, 2017, 121, 15719-15727.

25 O. F. Schirmer, V. Wittwer, G. Baur and G. Brandt, J. Electrochem. Soc., 1977, 124, 749-753.

26 R. Baetens, B. P. Jelle and A. Gustavsen, Sol. Energy Mater. Sol. Cells, 2010, 94, 87-105.

27 M. Tian and C. Shang, Int. J. Hydrogen Energy, 2019, 44, 338-344.

28 Y. Liu, J. Chen, L. Peng, N. Deng and W. Ding, Int. J. Hydrogen Energy, 2019, 44, 15205-15217.

29 Y. Liu, J. Chen, L. Peng, J. Han, N. Deng, W. Ding and W. Chen, Mater. Des., 2018, 144, 256-262.

30 J. S. E. M. Svensson and C. G. Granqvist, Thin Solid Films, 1985, 126, 31-36.

31 M. Antinucci, B. Chevalier and A. Ferriolo, Sol. Energy Mater. Sol. Cells, 1995, 39, 271-287.

32 M. Gratzel, Nature, 2001, 409, 575-576.

33 A. Llordes, G. Garcia, J. Gazquez and D. J. Milliron, Nature, 2013, 500, 323-326.

34 H. Chou, A. Nguyen, A. Chortos, J. W. F. To, C. Lu, J. Mei, T. Kurosawa, W. Bae, J. B. H. Tok and Z. Bao, Nat. Commun., 2015, 6, 8011.

35 G. Cai, J. Tu, C. Gu, J. Zhang, J. Chen, D. Zhou, S. J. Shi and X. Wang, J. Mater. Chem., 2013, 1, 4286-4292.

36 G. Cai, J. Tu, J. Zhang, Y. J. Mai, Y. Lu, C. Gu and X. Wang, Nanoscale, 2012, 4, 5724-5730.

37 G. Cai, J. Wang and P. S. Lee, Acc. Chem. Res., 2016, 49, 1469-1476.

38 W. Wu, M. Wang, J. Ma, Y. Cao and Y. Deng, Adv. Electron. Mater., 2018, 4, 1800185.

39 C. L. Bird and A. T. Kuhn, Chem. Soc. Rev., 1981, 10, 49-82. 40 G. Garcia, R. Buonsanti, A. Llordes, E. L. Runnerstrom, A. Bergerud and D. J. Milliron, Adv. Opt. Mater., 2013, 1, 215-220.

41 J. Kim, G. K. Ong, Y. Wang, G. Leblanc, T. E. Williams, T. M. Mattox, B. A. Helms and D. J. Milliron, Nano Lett., 2015, 15, 5574-5579.

42 C. J. Dahlman, Y. Tan, M. A. Marcus and D. J. Milliron, J. Am. Chem. Soc., 2015, 137, 9160-9166.

43 M. Pugliese, F. Bisconti, A. Rizzo, S. Colella, C. T. Prontera, G. Gigli, V. Maiorano and P. Cossari, ACS Appl. Energy Mater., 2020, 3, 10453-10462.

44 C. J. Barile, D. J. Slotcavage and M. D. McGehee, Chem. Mater., 2016, 28, 1439-1445. 
45 Y. Zhai, Y. Wang, X. Zhu, Z. Xing, S. Qi, S. Wang, Y. Han and Z. Chen, Macromolecules, 2021, 54, 5249-5259.

46 X. Lv, W. Li, M. Ouyang, Y. Zhang, D. S. Wright and C. Zhang, J. Mater. Chem. C, 2017, 5, 12-28.

47 H.-J. Yen and G.-S. Liou, Prog. Polym. Sci., 2019, 89, 250-287.

48 C. K. Lo, D. E. Shen and J. R. Reynolds, Macromolecules, 2019, 52, 6773-6779.

49 H. Yu, S. Shao, L. Yan, H. Meng, Y. He, C. Yao, P. Xu, X. Zhang, W. Hu and W. Huang, J. Mater. Chem. C, 2016, 4, 2269-2273.

50 C. N. Us and M. Icli Ozkut, Macromolecules, 2016, 49, 3009-3015.

51 H.-J. Yen and G.-S. Liou, Polym. Chem., 2018, 9, 3001-3018.

52 S. Schraff, Y. Sun and F. Pammer, Macromolecules, 2018, 51, 5323-5335.

53 Y. Han, Z. Xing, P. Ma, S. Li, C. Wang, Z. Jiang and Z. Chen, ACS Appl. Mater. Interfaces, 2020, 12, 7529-7538.

54 Y. Han, Y. Lin, D. Sun, Z. Xing, Z. Jiang and Z. Chen, Dyes Pigm., 2019, 163, 40-47.

55 A. Chaudhary, D. K. Pathak, M. Tanwar, J. Koch, H. Pfnür and R. Kumar, J. Mater. Chem. C, 2020, 8, 1773-1780.

56 H. Oh, J. K. Lee, Y. M. Kim, T. Y. Yun, U. Jeong and H. C. Moon, ACS Appl. Mater. Interfaces, 2019, 11, 45959-45968.

57 T. Y. Yun and H. C. Moon, Org. Electron., 2018, 56, 178-185.

58 Y. Alesanco, J. Palenzuela, A. Viñuales, G. Cabañero, H. J. Grande and I. Odriozola, ChemElectroChem, 2015, 2, 218-223.

59 J.-W. Kim and J.-M. Myoung, Adv. Funct. Mater., 2019, 29, 1808911.

60 M. Kim, Y. M. Kim and H. C. Moon, $R S C A d v .$, 2020, 10, 394-401.

61 G. K. Pande, N. Kim, J. H. Choi, G. Balamurugan, H. C. Moon and J. S. Park, Sol. Energy Mater. Sol. Cells, 2019, 197, 25-31.

62 H. C. Moon, C.-H. Kim, T. P. Lodge and C. D. Frisbie, ACS Appl. Mater. Interfaces, 2016, 8, 6252-6260.

63 J.-W. Kim and J.-M. Myoung, Adv. Funct. Mater., 2019, 29, 1808911.

64 Y. R. In, Y. M. Kim, Y. Lee, W. Y. Choi, S. H. Kim, S. W. Lee and H. C. Moon, ACS Appl. Mater. Interfaces, 2020, 12, 30635-30642.

65 R. J. Mortimer, A. L. Dyer and J. R. Reynolds, Displays, 2006, 27, 2-18.

66 J. Wang, L. Zhang, L. Yu, Z. Jiao, H. Xie, X. W. Lou and X. W. Sun, Nat. Commun., 2014, 5, 4921.

67 M. Guo, Q. Yu, X. Wang, W. Xu, Y. Wei, Y. Ma, J. Yu and B. Ding, ACS Appl. Mater. Interfaces, 2021, 13, 5634-5644.

68 S.-W. Oh, S.-M. Nam, S.-H. Kim, T.-H. Yoon and W. S. Kim, ACS Appl. Mater. Interfaces, 2021, 13, 5028-5033.

69 X. Li, W. Xie, C. Sui and P.-C. Hsu, ACS Materials Lett., 2020, 2, 1624-1643.

70 Y. Zhao, X. Zhang, X. Chen, W. Li, L. Wang, F. Ren, J. Zhao, F. Endres and Y. Li, ACS Sustainable Chem. Eng., 2020, 8, 11658-11666.
71 X. Huo, H. Zhang, W. Shen, X. Miao, M. Zhang and M. Guo, J. Mater. Chem. A, 2019, 7, 16867-16875.

72 Y.-C. Her and C.-C. Chang, CrystEngComm, 2014, 16, 5379-5386.

73 K.-W. Kim, Y. M. Kim, X. Li, T. Ha, S. H. Kim, H. C. Moon and S. W. Lee, Nanomaterials, 2020, 10, 821.

74 P.-W. Chen, C.-T. Chang, T.-F. Ko, S.-C. Hsu, K.-D. Li and J.-Y. Wu, Sci. Rep., 2020, 10, 8430.

75 E. A. Rojas-Gonzalez and G. A. Niklasson, arXiv.org, e-Print Arch., Phys., 2020, 1-10.

76 S. Mujawar, B. Dhale and P. S. Patil, Mater. Today: Proc., 2020, 23, 430-436.

77 S. R. Bathe and P. S. Patil, Mater. Sci. Eng., B, 2020, 257, 114542.

78 E. Hopmann, B. N. Carnio, C. J. Firby, B. Y. Shahriar and A. Y. Elezzabi, Nano Lett., 2021, 21, 1955-1961.

79 D. T. Gillaspie, R. C. Tenent and A. C. Dillon, J. Mater. Chem., 2010, 20, 9585-9592.

80 O. Lev, Z. Wu, S. Bharathi, V. Glezer, A. Modestov, J. Gun, L. Rabinovich and S. Sampath, Chem. Mater., 1997, 9, 2354-2375.

81 J. Yan, T. Wang, G. Wu, W. Dai, N. Guan, L. Li and J. Gong, Adv. Mater., 2015, 27, 1580-1586.

82 S. A. Agnihotry, Bull. Electrochem., 1996, 12, 707-712.

83 G. Cai, A. L.-S. Eh, L. Ji and P. S. Lee, Adv. Sustainable Syst., 2017, 1, 1700074.

84 G. Yang, Y.-M. Zhang, Y. Cai, B. Yang, C. Gu and S. X.-A. Zhang, Chem. Soc. Rev., 2020, 49, 8687-8720.

85 K. Zhou, H. Wang, S. Zhang, J. Jiu, J. Liu, Y. Zhang and H. Yan, J. Mater. Sci., 2017, 52, 12783-12794.

86 G. Cai, M. Cui, V. Kumar, P. Darmawan, J. Wang, X. Wang, A. L. Eh, K. Qian and P. S. Lee, Chem. Sci., 2016, 7, 1373-1382.

87 V. Rai, R. S. Singh, D. J. Blackwood and D. Zhili, Adv. Eng. Mater., 2020, 22, 2000082.

88 S. Cong, F. Geng and Z. Zhao, Adv. Mater., 2016, 28, 10518-10528.

89 W. He, Y. Liu, Z. Wan and C. Jia, RSC Adv., 2016, 6, 68997-69006.

90 S. Bogati, A. Georg and W. Graf, Sol. Energy Mater. Sol. Cells, 2017, 159, 395-404.

91 K. S. Usha, R. Sivakumar, C. Sanjeeviraja, V. Sathe, V. Ganesan and T. Y. Wang, RSC Adv., 2016, 6, 79668-79680.

92 M. Kateb, S. Safarian, M. Kolahdouz, M. Fathipour and V. Ahamdi, Sol. Energy Mater. Sol. Cells, 2016, 145, 200-205.

93 J. Zhang, Z. Chen, L. Yang, F. Hu, G. Yu, J. Yin and S. Liu, Dyes Pigm., 2017, 136, 168-174.

94 J. Zhang, G. Liu, X.-Y. Wang, G.-A. Yu, J. Yin and S.-H. Liu, Dyes Pigm., 2017, 143, 416-426.

95 B. Chiou, H. Lee, Y. Jang, Z. Yang, Y. Wang, M. Sarma, H. Su and K. Wong, Org. Electron., 2017, 48, 248-253.

96 W. Zhang, W. Ju, X. Wu, Y. Wang, Q. Wang, H. Zhou, S. Wang and C. Hu, Appl. Surf. Sci., 2016, 367, 542-551.

97 G. Nie, L. Wang and C. Liu, J. Mater. Chem. C, 2015, 3, 11318-11325. 
98 E. C. Cho, C. W. Changjian, Y. Hsiao, K. C. Lee and J. Huang, Sol. Energy Mater. Sol. Cells, 2016, 150, 43-50.

99 Y. Ji, C. Zhang, H. Niu, X. Zhao, C. Wang, C. Qin, W. Wang and X. Bai, Dyes Pigm., 2016, 125, 106-115.

100 N. Deforest, A. Shehabi, G. Garcia, J. B. Greenblatt, E. Masanet, E. S. Lee, S. Selkowitz and D. J. Milliron, Build. Environ., 2013, 61, 160-168.

101 S.-H. Lee, R. Deshpande, P. A. Parilla, K. M. Jones, B. To, A. H. Mahan and A. C. Dillon, Adv. Mater., 2006, 18, 763-766.

102 P. Yang, P. Sun, Z. Chai, L. Huang, X. Cai, S. Tan, J. Song and W. Mai, Angew. Chem., 2014, 53, 11935-11939.

103 R. Wen, C. Granqvist and G. A. Niklasson, Nat. Mater., 2015, 14, 996-1001.

104 V. K. Thakur, G. Ding, J. Ma, P. S. Lee and X. Lu, Adv. Mater., 2012, 24, 4071-4096.

105 E. Hwang, S. Seo, S. Bak, H. Lee, M. Min and H. Lee, Adv. Mater., 2014, 26, 5129-5136.

106 A. A. Argun, P. Aubert, B. C. Thompson, I. Schwendeman, C. L. Gaupp, J. K. Hwang, N. J. Pinto, D. B. Tanner, A. G. Macdiarmid and J. R. Reynolds, Chem. Mater., 2004, 16, 4401-4412.

107 H. Wei, X. Yan, S. Wu, Z. Luo, S. Wei and Z. Guo, J. Phys. Chem. C, 2012, 116, 25052-25064.

108 M. Cui, W. S. Ng, X. Wang, P. Darmawan and P. S. Lee, Adv. Funct. Mater., 2015, 25, 401-408.

109 J. H. Jensen, M. Hosel, A. L. Dyer and F. C. Krebs, Adv. Funct. Mater., 2015, 25, 2073-2090.

110 P. M. Beaujuge and J. R. Reynolds, Chem. Rev., 2010, 110, 268-320.

111 J. He, L. You and J. Mei, ACS Appl. Mater. Interfaces, 2017, 9, 34122-34130.

112 H. Oh, D. G. Seo, T. Y. Yun, C. Y. Kim and H. C. Moon, ACS Appl. Mater. Interfaces, 2017, 9, 7658-7665.

113 X. Liu, L. Kong, H. Du, Y. Zhang, J. Zhao and Y. Xie, Org. Electron., 2019, 64, 223-235.

114 G. Cai, P. Darmawan, M. Cui, J. Wang, J. Chen, S. Magdassi and P. S. Lee, Adv. Eng. Mater., 2016, 6, 1501882.

115 C. Guillén and J. Herrero, J. Mater. Sci. Technol., 2021, 78, 223-228.

116 H. Yu, J. Guo, C. Wang, J. Zhang, J. Liu, X. Zhong, G. Dong and X. Diao, Electrochim. Acta, 2019, 318, 644-650.

117 R. Baetens, B. P. Jelle and A. Gustavsen, Sol. Energy Mater. Sol. Cells, 2010, 94, 87-105.

118 T. V. Nguyen, K. A. Huynh, Q. V. Le, H. Kim, S. H. Ahn and S. Y. Kim, Int. J. Energy Res., 2021, 45, 8061-8072.

119 J. Wang, Y. Lu, H. Li, J. Liu and S. Yu, J. Am. Chem. Soc., 2017, 139, 9921-9926.

120 K.-W. Kim, T. Y. Yun, S.-H. You, X. Tang, J. Lee, Y. Seo, Y.-T. Kim, S. H. Kim, H. C. Moon and J. K. Kim, NPG Asia Mater., 2020, 12, 84.

121 Z. Wang, W. Gong, X. Wang, Z. Chen, X. Chen, J. Chen, H. Sun, G. Song, S. Cong, F. Geng and Z. Zhao, ACS Appl. Mater. Interfaces, 2020, 12, 33917-33925.

122 H. Gu, C. Guo, S. Zhang, L. Bi, T. Li, T. Sun and S. Liu, ACS Nano, 2018, 12, 559-567.
123 S. Heo, J. Kim, G. K. Ong and D. J. Milliron, Nano Lett., 2017, 17, 5756-5761.

124 W. Dong, Y. Lv, L. Xiao, Y. Fan, N. Zhang and X. Liu, ACS Appl. Mater. Interfaces, 2016, 8, 33842-33847.

125 Y. Yin, C. Lan, H. Guo and C. Li, ACS Appl. Mater. Interfaces, 2016, 8, 3861-3867.

126 J. Liu, O. Margeat, W. Dachraoui, X. Liu, M. Fahlman and J. Ackermann, Adv. Funct. Mater., 2014, 24, 6029-6037.

127 X. Dang, X. Jiang, T. Zhang and H. Zhao, Chin. J. Chem., 2021, 39, 1706-1715.

128 D. Chatzikyriakou, N. Krins, B. Gilbert, P. Colson, J. Dewalque, J. Denayer, R. Cloots and C. Henrist, Electrochim. Acta, 2014, 137, 75-82.

129 P. R. Bueno, R. C. Faria, C. O. Avellaneda, E. R. Leite and L. O. S. Bulhões, Solid State Ionics, 2003, 158, 415-426.

130 C. Bechinger, M. S. Burdis and J. Zhang, Solid State Commun., 1997, 101, 753-756.

131 S. Lee, H. M. Cheong, C. E. Tracy, A. Mascarenhas, A. W. Czanderna and S. K. Deb, Appl. Phys. Lett., 1999, 75, 1541-1543.

132 A. Al Mohammad and M. Gillet, Thin Solid Films, 2002, 408, 302-309.

133 P. A. Shinde and S. C. Jun, ChemSusChem, 2020, 13, 11-38. 134 Z. Shen, Z. Zhao, J. Wen, J. Qian, Z. Peng and X. Fu, J. Nanomater., 2018, 2018, 1-12.

135 T. Masuda and H. Yao, J. Phys. Chem. C, 2020, 124, 15460-15467.

136 H. Yu, J. Guo, C. Wang, J. Zhang, J. Liu, G. Dong, X. Zhong and X. Diao, Electrochim. Acta, 2020, 332, 135504.

137 X. Zeng, Y. Zhou, S. Ji, H. Luo, H. Yao, X. Huang and P. Jin, J. Mater. Chem. C, 2015, 3, 8050-8060.

138 W. Zhang, L. Yue, F. Zhang, Q. Zhang, X. Gui, R. Guan, G. Hou and N. Xu, J. Mater. Chem. A, 2015, 3, 6102-6109.

139 K. Manthiram and A. P. Alivisatos, J. Am. Chem. Soc., 2012, 134, 3995-3998.

140 W. Huang and M. A. El-Sayed, Eur. Phys. J. Spec. Top., 2008, 153, 325-333.

141 X. Wang, F. Wang, Y. Sang and H. Liu, Adv. Enegy Mater., 2017, 7, 1700473.

142 G. L. Frey, A. Rothschild, J. Sloan, R. Rosentsveig, R. Popovitz-Biro and R. Tenne, J. Solid State Chem., 2001, 162, 300-314.

143 C. Santato, M. Odziemkowski, M. Ulmann and J. Augustynski, J. Am. Chem. Soc., 2001, 123, 10639-10649.

144 T. Arai, M. Horiguchi, M. Yanagida, T. Gunji, H. Sugihara and K. Sayama, J. Phys. Chem. C, 2009, 113, 6602-6609.

145 T. Paik, M. Cargnello, T. R. Gordon, S. Zhang, H. Yun, J. D. Lee, H. Y. Woo, S. J. Oh, C. R. Kagan and P. Fornasiero, ACS Energy Lett., 2018, 3, 1904-1910.

146 M. M. Natile, F. Tomaello and A. Glisenti, Chem. Mater., 2005, 17, 3270-3280.

147 T. Onfroy, G. Clet and M. Houalla, J. Phys. Chem. B, 2005, 109, 3345-3354.

148 Z. Chen, Q. Wang, H. Wang, L. Zhang, G. Song, L. Song, J. Hu, H. Wang, J. Liu and M. Zhu, Adv. Mater., 2013, 25, 2095-2100. 
149 S. Zeb, G. X. Sun, Y. Nie, Y. Cui and X. C. Jiang, Sens. Actuators, B, 2020, 321, 128439.

150 B. Xiao, Q. Zhao, C. Xiao, T. Yang, P. Wang, F. Wang, X. Chen and M. Zhang, CrystEngComm, 2015, 17, 5710-5716.

151 S. Bai, K. Zhang, X. Shu, S. Chen, R. Luo, D. Li and A. Chen, CrystEngComm, 2014, 16, 10210-10217.

152 S. Zeb, X. J. Peng, G. Z. Yuan, X. X. Zhao, C. Y. Qin, G. X. Sun, Y. Nie, Y. Cui and X. C. Jiang, Sens. Actuators, $B, 2020,305,127435$.

153 X. Yang, V. Salles, Y. V. Kaneti, M. Liu, M. Maillard, C. Journet, X. Jiang and A. Brioude, Sens. Actuators, B, 2015, 220, 1112-1119.

154 Y. V. Kaneti, J. Moriceau, M. Liu, Y. Yuan, Q. M. Zakaria, X. Jiang and A. Yu, Sens. Actuators, B, 2015, 209, 889-897.

155 S. Balaji, Y. Djaoued, A.-S. Albert, R. Z. Ferguson and R. Brüning, Chem. Mater., 2009, 21, 1381-1389.

156 H. Zheng, J. Z. Ou, M. S. Strano, R. B. Kaner, A. Mitchell and K. Kalantar-zadeh, Adv. Funct. Mater., 2011, 21, 2175-2196.

157 S. Shi, F. Teng, W. Hao, W. Gu, Z. Yang and F. Zhao, Inorg. Chem., 2019, 58, 9161-9168.

158 T. F. Chala, C.-M. Wu, M.-H. Chou, M. B. Gebeyehu and K.-B. Cheng, Nanomaterials, 2017, 7, 191.

159 K. Kalantarzadeh, A. Vijayaraghavan, M. H. Ham, H. Zheng, M. Breedon and M. S. Strano, Chem. Mater., 2010, 22, 5660-5666.

160 L. M. Kuti, S. S. Bhella and V. Thangadurai, Inorg. Chem., 2009, 48, 6804-6811.

161 J. B. Mitchell, W. C. Lo, A. Genc, J. LeBeau and V. Augustyn, Chem. Mater., 2017, 29, 3928-3937.

162 D. Muñoz-Santiburcio, C. Wittekindt and D. Marx, Nat. Commun., 2013, 4, 2349.

163 Z. Wang, W. Gong, X. Wang, Z. Chen, X. Chen, J. Chen, H. Sun, G. Song, S. Cong, F. Geng and Z. Zhao, ACS Appl. Mater. Interfaces, 2020, 12, 33917-33925.

164 Z. Bi, X. Li, Y. Chen, X. He, X. Xu and X. Gao, ACS Appl. Mater. Interfaces, 2017, 9, 29872-29880.

165 D. B. Migas, V. L. Shaposhnikov and V. E. Borisenko, J. Appl. Phys., 2010, 108, 093714.

166 H. Kaper, I. Djerdj, S. Gross, H. Amenitsch, M. Antonietti and B. M. Smarsly, Phys. Chem. Chem. Phys., 2015, 17, 18138-18145.

167 C. Guo, S. Yin and T. Sato, J. Am. Ceram. Soc., 2012, 95, 1634-1639.

168 C. Guo, S. Yin, M. Yan and T. Sato, J. Mater. Chem., 2011, 21, 5099-5105.

169 M. B. Johansson, G. Baldissera, I. Valyukh, C. Persson, H. Arwin, G. A. Niklasson and L. Osterlund, J. Phys.: Condens. Matter, 2013, 25, 205502.

170 A. Hjelm, C. G. Granqvist and J. M. Wills, Phys. Rev. B: Condens. Matter Mater. Phys., 1996, 54, 2436-2445.

171 M. Saleem, M. F. Alkuhaili, S. M. A. Durrani, A. H. Y. Hendi, I. A. Bakhtiari and S. Ali, Int. J. Hydrogen Energy, 2015, 40, 12343-12351.

172 F. Gervais, Mater. Sci. Eng., R, 2002, 39, 29-92.
173 A. M. Smith and S. Nie, Acc. Chem. Res., 2010, 43, 190-200.

174 R. Chatten, A. V. Chadwick, A. Rougier and P. J. D. Lindan, J. Phys. Chem. B, 2005, 109, 3146-3156.

175 H. Kamal, A. A. Akl and K. Abdelhady, Phys. B, 2004, 349, 192-205.

176 C. G. Granqvist, Sol. Energy Mater. Sol. Cells, 2000, 60, 201-262.

177 J. S. E. M. Svensson and C. G. Granqvist, Appl. Phys. Lett., 1984, 45, 828-830.

178 S. Lee, H. Cheong, C. E. Tracy, A. Mascarenhas, A. W. Czanderna and S. K. Deb, Appl. Phys. Lett., 1999, 75, 1541-1543.

179 S.-H. Lee, H. M. Cheong, C. E. Tracy, A. Mascarenhas, A. W. Czanderna and S. K. Deb, Appl. Phys. Lett., 1999, 75, 1541-1543.

180 P. R. Bueno, F. M. Pontes, E. R. Leite, L. O. S. Bulhoes, P. S. Pizani, P. N. Lisboafilho and W. H. Schreiner, J. Appl. Phys., 2004, 96, 2102-2109.

181 T. Ohtsuka, N. Goto and N. Sato, J. Electroanal. Chem., 1990, 287, 249-264.

182 J. G. Zhang, D. K. Benson, C. E. Tracy, S. K. Deb, A. W. Czanderna and C. Bechinger, ChemInform, 1997, 28, 2022-2026.

183 S. Lee, H. Cheong, C. E. Tracy, A. Mascarenhas, R. Pitts, G. Jorgensen and S. K. Deb, Electrochim. Acta, 2001, 46, 3415-3419.

184 C. Granqvist, M. A. Arvizu, I. B. Pehlivan, H. Y. Qu, R. Wen and G. A. Niklasson, Electrochim. Acta, 2018, 259, 1170-1182.

185 M. Deepa, A. K. Srivastava, K. N. Sood and S. A. Agnihotry, Nanotechnology, 2006, 17, 2625-2630.

186 M. Stolze, B. Camin, F. Galbert, U. Reinholz and L. K. Thomas, Thin Solid Films, 2002, 409, 254-264.

187 G. A. Niklasson, L. Berggren and A. Larsson, Sol. Energy Mater. Sol. Cells, 2004, 84, 315-328.

188 S. K. Deb, Sol. Energy Mater. Sol. Cells, 2008, 92, 245-258.

189 K. Thummavichai, Y. Xia and Y. Zhu, Prog. Mater. Sci., 2017, 88, 281-324.

190 J. Olejnicek, M. Brunclikova, S. Kment, Z. Hubicka, H. Kmentova, P. Ksirova, M. Cada, M. Zlamal and J. Krýsa, Chem. Eng. J., 2017, 318, 281-288.

191 S. K. Chong, C. F. Dee and S. A. Rahman, RSC Adv., 2015, 5, 2346-2353.

192 V. V. Kondalkar, S. S. Yang, P. S. Patil, S. Choudhury, P. N. Bhosale and K. K. Lee, Mater. Lett., 2017, 194, 102-106.

193 E. Khoo, P. S. Lee and J. Ma, J. Eur. Ceram. Soc., 2010, 30, 1139-1144.

194 C. Dulgerbaki, N. N. Maslakci, A. I. Komur and A. U. Oksuz, Mater. Res. Bull., 2015, 72, 70-76.

195 J. Zhang, J. Tu, X. Xia, X. Wang and C. Gu, J. Mater. Chem., 2011, 21, 5492-5498.

196 A. L.-S. Eh, A. W. M. Tan, X. Cheng, S. Magdassi and P. S. Lee, Energy Technol., 2018, 6, 33-45.

197 J.-Y. Li, Q. Xu, G. Li, Y.-X. Yin, L.-J. Wan and Y.-G. Guo, Mater. Chem. Front., 2017, 1, 1691-1708. 
198 Z. Meng, R. M. Stolz, L. Mendecki and K. A. Mirica, Chem. Rev., 2019, 119, 478-598.

199 R. G. Gordon, S. Barry, J. T. Barton and R. N. R. BroomhallDillard, Thin Solid Films, 2001, 392, 231-235.

200 C. S. Blackman and I. P. Parkin, Chem. Mater., 2005, 17, 1583-1590.

201 E. Brescacin, M. Basato and E. Tondello, Chem. Mater., 1999, 11, 314-323.

202 N. C. Ou, D. C. Bock, X. Su, D. Craciun, V. Craciun and L. McElwee-White, ACS Appl. Mater. Interfaces, 2019, 11, 28180-28188.

203 V. Jadkar, A. Pawbake, R. Waykar, A. Jadhavar, A. Date, D. Late, H. Pathan, S. Gosavi and S. Jadkar, Phys. Status Solidi A, 2017, 214, 1600717.

204 Z. S. Houweling, J. W. Geus and R. E. I. Schropp, Chem. Vap. Deposition, 2010, 16, 179-184.

205 K. A. Gesheva and T. Ivanova, Chem. Vap. Deposition, 2006, 12, 231-238.

206 A. Donnadieu, D. Davazoglou and A. Abdellaoui, Thin Solid Films, 1988, 164, 333-338.

207 T. Ivanova, K. A. Gesheva, M. Kalitzova, B. Marsen, B. Cole and E. L. Miller, Mater. Sci. Eng., B, 2007, 142, 126-134.

208 B. Zheng, W. Zheng, Y. Jiang, S. Chen, D. Li, C. Ma, X. Wang, W. Huang, X. Zhang, H. Liu, F. Jiang, L. Li, X. Zhuang, X. Wang and A. Pan, J. Am. Chem. Soc., 2019, 141, 11754-11758.

209 S. Hoseinzadeh, R. Ghasemiasl, A. Bahari and A. Ramezani, J. Electron. Mater., 2018, 47, 3552-3559.

210 A. Esmail, H. Hashem, S. Soltan, M. Hammam and A. Ramadan, Phys. Status Solidi A, 2017, 214, 1600478.

211 K. W. Kim, Y. M. Kim, X. Li, T. Ha, S. H. Kim, H. C. Moon and S. W. Lee, Nanomaterials, 2020, 10, 821.

212 B. B. Lakshmi, C. J. Patrissi and C. R. Martin, Chem. Mater., 1997, 9, 2544-2550.

213 Z. Ling, K. Liu, Q. Zou, Q. Li, K.-Q. Zhang, Z. Cui, W. Yuan and Y. Liu, RSC Adv., 2018, 8, 28581-28587.

214 S. Adhikari, R. Swain, D. Sarkar and G. Madras, Mol. Catal., 2017, 432, 76-87.

215 E. Bica, L. E. Muresan, L. Barbu-Tudoran, E. Indrea, I. C. Popescu and E.-J. Popovici, Stud. Univ. Babes-Bolyai, Chem., 2009, 54, 15-22.

216 C.-Y. Kim, M. Lee, S.-H. Huh and E.-K. Kim, J. Sol-Gel Sci. Technol., 2010, 53, 176-183.

217 B. W. C. Au, K. Chan, W. L. Pang, C. L. Lee and A. H. Mustafa, Solid State Phenom., 2018, 280, 71-75.

218 S. Zhang, S. Chen, F. Hu, R. Xu, B. Yan, M. Jiang, Y. Gu, F. Yang and Y. Cao, Sol. Energy Mater. Sol. Cells, 2019, 200, 109951.

219 A. Bellingham, N. Bromhead and A. Fontecchio, Materials, 2017, 10, 594.

220 V. V. Kondalkar, S. S. Yang, P. S. Patil, S. Choudhury, P. N. Bhosale and K. Lee, Mater. Lett., 2017, 194, 102-106.

221 O. Prakash, V. Saxena, S. Choudhury, Tanvi, A. Singh, A. K. Debnath, A. Mahajan, K. P. Muthe and D. K. Aswal, Electrochim. Acta, 2019, 318, 405-412.
222 V. V. Kondalkar, S. S. Mali, R. R. Kharade, R. M. Mane, P. S. Patil, C. K. Hong, J. H. Kim, S. Choudhury and P. N. Bhosale, RSC Adv., 2015, 5, 26923-26931.

223 Z. Asghari and H. A. Zad, arXiv.org, e-Print Arch., Phys., 2019, 1-15.

224 A. Chemseddine, M. Henry and J. Livage, Revue de Chirnie rninérale, 1984, $1,487$.

225 B. Zhao, X. Zhang, G. Dong, H. Wang and H. Yan, Ionics, 2015, 21, 2879-2887.

226 T. He and J. Yao, Prog. Mater. Sci., 2006, 51, 810-879.

227 K. Bange and T. Gambke, Adv. Mater., 1990, 2, 10-16.

228 L. G. Alexander, KR Pat., 2011132858A, 2011.

229 J. Wang, E. Khoo, P. S. Lee and J. Ma, J. Phys. Chem. C, 2009, 113, 9655-9658.

230 J. Wang, E. Khoo, P. S. Lee and J. Ma, J. Phys. Chem. C, 2008, 112, 14306-14312.

231 R. R. Kharade, S. S. Mali, S. S. Mohite, V. V. Kondalkar, P. S. Patil and P. N. Bhosale, Electroanalysis, 2014, 26, 2388-2397.

232 Y. J. Li, Z. F. Liu, X. P. Liang, J. Ya, T. Cui and Z. C. Liu, Mater. Technol., 2014, 29, 341-349.

233 M. Alsawafta, Y. M. Golestani, T. Phonemac, S. Badilescu, V. Stancovski and V.-V. Truong, J. Electrochem. Soc., 2014, 161, H276-H283.

234 K. Paipitak, W. Techitdheera, S. Porntheeraphat and W. Pecharapa, Energy Procedia, 2013, 34, 689-696.

235 R. R. Kharade, S. S. Mali, S. P. Patil, K. R. Patil, M. G. Gang, P. S. Patil, J. H. Kim and P. N. Bhosale, Electrochim. Acta, 2013, 102, 358-368.

236 Y. Djaoued, S. Balaji and N. Beaudoin, J. Sol-Gel Sci. Technol., 2013, 65, 374-383.

237 K. Paipitak, J. Rattanarak, D. Pakdeeyingyong, W. Techitdheera, S. Porntheeraphat and W. Pecharapa, Adv. Mater. Res., 2012, 528, 249-253.

238 P. J. Wojcik, A. S. Cruz, L. Santos, L. Pereira, R. Martins and E. Fortunato, J. Mater. Chem., 2012, 22, 13268-13278.

239 C.-L. Wu, C.-K. Wang, C.-K. Lin, S.-C. Wang and J.-L. Huang, Surf. Coat. Technol., 2013, 231, 403-407.

240 J. Zhang, J.-p. Tu, D. Zhang, Y.-q. Qiao, X.-h. Xia, X.-l. Wang and C.-d. Gu, J. Mater. Chem., 2011, 21, 17316-17324.

241 T. V. Nguyen, K. A. Huynh, Q. V. Le, H. Kim, S. H. Ahn and S. Y. Kim, Int. J. Energy Res., 2021, 45, 8061-8072.

242 M. H. Azarian and J. Wootthikanokkhan, J. Appl. Polym. Sci., 2021, 138, 49863.

243 P. Jittiarporn, L. Sikong, K. Kooptarnond, W. Taweepreda, S. Stoenescu, S. Badilescu and V.-V. Truong, Surf. Coat. Technol., 2017, 327, 66-74.

244 X. L. Sun, A. H. Chen, H. Z. Zhang and H. Cao, Adv. Mater. Res., 2009, 843-846.

245 Y. Djaoued, S. Balaji and N. Beaudoin, J. Sol-Gel Sci. Technol., 2013, 65, 374-383.

246 J. Wang, G. Liu and Y. Du, Mater. Lett., 2003, 57, 3648-3652.

247 Y. Shi, M. Sun, Y. Zhang, J. Cui, X. Shu, Y. Wang, Y. Qin, J. Liu, H. H. Tan and Y. Wu, ACS Appl. Mater. Interfaces, 2020, 12, 32658-32665. 
248 S. S. Kalagi, S. S. Mali, D. S. Dalavi, A. I. Inamdar, H. Im and P. S. Patil, Electrochim. Acta, 2012, 85, 501-508.

249 A. I. Inamdar, Y. S. Kim, B. U. Jang, H. Im, W. Jung, D.-Y. Kim and H. Kim, Thin Solid Films, 2012, 520, 5367-5371.

250 F. C. Cheong, B. Varghese, Y. Zhu, E. P. S. Tan, L. Dai, V. B. C. Tan, C. T. Lim and C. H. Sow, J. Phys. Chem. C, 2007, 111, 17193-17199.

251 X. Liu, Y. He, S. Wang, Q. Zhang and M. Song, Int. J. Refract. Met. Hard Mater., 2012, 34, 47-52.

252 G. Duan, L. Chen, Z. Jing, P. De Luna, L. Wen, L. Zhang, L. Zhao, J. Xu, Z. Li, Z. Yang and R. Zhou, Chem. Res. Toxicol., 2019, 32, 1357-1366.

253 L. Wen, L. Chen, S. Zheng, J. Zeng, G. Duan, Y. Wang, G. Wang, Z. Chai, Z. Li and M. Gao, Adv. Mater., 2016, 28, 5072-5079.

254 G. Prusty, J. T. Lee, S. Seifert, B. B. Muhoberac and R. Sardar, J. Am. Chem. Soc., 2020, 142, 5938-5942.

255 S. Cong, Y. Tian, Q. Li, Z. Zhao and F. Geng, Adv. Mater., 2014, 26, 4260-4267.

256 G. Cai, P. Darmawan, M. Cui, J. Wang, J. Chen, S. Magdassi and P. S. Lee, Adv. Energy Mater., 2016, 6, 1501882.

257 B. J.-W. Liu, J. Zheng, J.-L. Wang, J. Xu, H.-H. Li and S.-H. Yu, Nano Lett., 2013, 13, 3589-3593.

258 J. Zhang, J.-p. Tu, X.-h. Xia, X.-l. Wang and C.-d. Gu, J. Mater. Chem., 2011, 21, 5492-5498.

259 Z. Jiao, J. Wang, L. Ke, X. Liu, H. V. Demir, M. F. Yang and X. W. Sun, Electrochim. Acta, 2012, 63, 153-160.

260 J. Pan, Y. Wang, R. Zheng, M. Wang, Z. Wan, C. Jia, X. Weng, J. Xie and L. Deng, J. Mater. Chem. A, 2019, 7, 13956-13967.

261 N. Y. Bhosale, S. S. Mali, C. K. Hong and A. V. Kadam, Electrochim. Acta, 2017, 246, 1112-1120.

262 G.-f. Cai, J.-p. Tu, D. Zhou, L. Li, J.-h. Zhang, X.-l. Wang and C.-d. Gu, CrystEngComm, 2014, 16, 6866-6872.

263 Y. Li, W. A. McMaster, H. Wei, D. Chen and R. A. Caruso, ACS Appl. Nano Mater., 2018, 1, 2552-2558.

264 G. Li, S. Zhang, C. Guo and S. Liu, Nanoscale, 2016, 8, 9861-9868.

265 Y. Sun, W. Wang, J. Qin, D. Zhao, B. Mao, Y. Xiao and M. Cao, Electrochim. Acta, 2016, 187, 329-339.

266 V. V. Kondalkar, R. R. Kharade, S. S. Mali, R. M. Mane, P. B. Patil, P. S. Patil, S. Choudhury and P. N. Bhosale, Superlattices Microstruct., 2014, 73, 290-295.

267 B. Wen-Cheun, Au, A. Tamang, D. Knipp and K.-Y. Chan, Opt. Mater., 2020, 108, 110426.

268 T. Dhandayuthapani, R. Sivakumar, D. Zheng, H. Xu, R. Ilangovan, C. Sanjeeviraja and J. Lin, Mater. Sci. Semicond. Process., 2020, 105515, DOI: 10.1016/j.mssp.2020. 105515.

269 W. Cheng, E. Baudrin, B. Dunn and J. I. Zink, J. Mater. Chem., 2001, 11, 92-97.

270 Y.-T. Park, S.-H. Lee and K.-T. Lee, Ceram. Int., 2020, 46, 29052-29059.

271 P. J. Wojcik, L. Pereira, R. Martins and E. Fortunato, ACS Comb. Sci., 2014, 16, 5-16.
272 G. Cai, X. Cheng, M. Layani, A. W. M. Tan, S. Li, A. L.-S. Eh, D. Gao, S. Magdassi and P. S. Lee, Nano Energy, 2018, 49, 147-154.

273 S. J. Yoo, Y. H. Jung, J. W. Lim, H. G. Choi, D. K. Kim and Y.-E. Sung, Sol. Energy Mater. Sol. Cells, 2008, 92, 179-183.

274 X. Li, T. Y. Yun, K.-W. Kim, S. H. Kim and H. C. Moon, ACS Appl. Mater. Interfaces, 2020, 12, 4022-4030.

275 G. Cai, M. Cui, V. Kumar, P. Darmawan, J. Wang, X. Wang, A. Lee-Sie Eh, K. Qian and P. S. Lee, Chem. Sci., 2016, 7, 1373-1382.

276 A. J. More, R. S. Patil, D. S. Dalavi, S. S. Mali, C. K. Hong, M. G. Gang, J. H. Kim and P. S. Patil, Mater. Lett., 2014, 134, 298-301.

277 C. Guo, S. Yin, Y. Huang, Q. Dong and T. Sato, Langmuir, 2011, 27, 12172-12178.

278 A. V. Kadam, N. Y. Bhosale, S. B. Patil, S. S. Mali and C. K. Hong, Thin Solid Films, 2019, 673, 86-93.

279 J. Chu, J. Lan, D. Lu, J. Ma, X. Wang, B. Wu, M. Gong, R. Zhang and S. Xiong, Micro Nano Lett., 2016, 11, 749-752.

280 S. Mathuri, M. M. Margoni, K. Ramamurthi, R. Ramesh Babu and V. Ganesh, Appl. Surf. Sci., 2018, 449, 77-91.

281 Y. Liang, Z. Liu, Y. Cheng and Z. Zhao, Integr. Ferroelectr., 2016, 172, 109-116.

282 C.-H. Lu, M. H. Hon, C.-Y. Kuan and I.-C. Leu, Jpn. J. Appl. Phys., 2014, 53, 06JG08.

283 S. Sun, Y. Zhao, Y. Xia, Z. Zou, G. Min and Y. Zhu, Nanotechnology, 2008, 19, 305709.

284 C. Lu, M. Hon, C. Kuan and I. Leu, J. Mater. Sci., 2015, 50, 5739-5745.

285 K. Huang, Q. Pan, F. Yang, S. Ni, X. Wei and D. He, J. Phys. D: Appl. Phys., 2008, 41, 155417.

286 J. Guo, Y. Shi, H. Zhou, X. Wang and T. Ma, RSC Adv., 2017, 7, 2051-2057.

287 S. Park, H. W. Shim, C. W. Lee, H. J. Song, J. C. Kim and D. W. Kim, Nano Res., 2016, 9, 633-643.

288 Z. Gu, T. Zhai, B. Gao, X. Sheng, Y. Wang, H. Fu, Y. Ma and J. Yao, J. Phys. Chem. B, 2006, 110, 23829-23836.

289 C. Guo, S. Yin, M. Yan, M. Kobayashi, M. Kakihana and T. Sato, Inorg. Chem., 2012, 51, 4763-4771.

290 F. Zheng, W. Man, M. Guo, M. Zhang and Q. Zhen, CrystEngComm, 2015, 17, 5440-5450.

291 L.-s. Li, J. Walda, L. Manna and A. P. Alivisatos, Nano Lett., 2002, 2, 557-560.

292 B. Sun and H. Sirringhaus, Nano Lett., 2005, 5, 2408-2413. 293 J. Xu, Y. Zhang, T. Zhai, Z. Kuang, J. Li, Y. Wang, Z. Gao, Y. Song and X. Xia, ACS Nano, 2018, 12, 6895-6903.

294 G. Garcia, R. Buonsanti, E. L. Runnerstrom, R. J. Mendelsberg, A. Llordes, A. Anders, T. J. Richardson and D. J. Milliron, Nano Lett., 2011, 11, 4415-4420.

295 K. Niinomi, S. Miyazawa, M. Hibino, N. Mizuno and S. Uchida, Inorg. Chem., 2017, 56, 15187-15193.

296 B. Xu, L. Xu, G. Gao, W. Guo and S. Liu, J. Colloid Interface Sci., 2009, 330, 408-414.

297 N. M. Vuong, D. Kim and H. Kim, J. Mater. Chem. C, 2013, 1, 3399-3407. 
298 C. Dulgerbaki, N. N. Maslakci, A. I. Komur and A. U. Oksuz, Electroanalysis, 2016, 28, 1873-1879.

299 H. Li, L. McRae and A. Y. Elezzabi, ACS Appl. Mater. Interfaces, 2018, 10, 10520-10527.

300 G. K. Dalapati, A. Kushwaha, M. Sharma, V. Suresh, S. Shannigrahi, S. Zhuk and S. Masudypanah, Prog. Mater. Sci., 2018, 95, 42-131.

301 J. Chang, R. Ahmed, H. Wang, H. Liu, R. Li, P. Wang and E. R. Waclawik, J. Phys. Chem. C, 2013, 117, 13836-13844.

302 L. Li, Z. Yu, W. Hu, C. Chang, Q. Chen and Q. Pei, Adv. Mater., 2011, 23, 5563-5567.

303 S. Shin, M. Yang, L. J. Guo and H. Youn, Small, 2013, 9, 4036-4044.

304 G. Leftheriotis, E. Koubli and P. Yianoulis, Sol. Energy Mater. Sol. Cells, 2013, 116, 110-119.

305 H. Li, Y. Lv, X. Zhang, X. Wang and X. Liu, Sol. Energy Mater. Sol. Cells, 2015, 136, 86-91.

306 E. Koubli, S. Tsakanikas, G. Leftheriotis, G. Syrrokostas and P. Yianoulis, Solid State Ionics, 2015, 272, 30-38.

307 L. Xiao, Y. Lv, W. Dong, N. Zhang and X. Liu, ACS Appl. Mater. Interfaces, 2016, 8, 27107-27114.

308 G. Leftheriotis, S. Papaefthimiou and P. Yianoulis, Sol. Energy Mater. Sol. Cells, 2000, 61, 107-112.

309 H. Najafi-Ashtiani, B. Akhavan, F. Jing and M. M. Bilek, ACS Appl. Mater. Interfaces, 2019, 11, 14871-14881.

310 A. Anders, Surf. Coat. Technol., 2014, 257, 308-325.

311 V. I. Klimov, S. A. Ivanov, J. Nanda, M. Achermann, I. Bezel, J. A. McGuire and A. Piryatinski, Nature, 2007, 447, 441-446.

312 X. Michalet, F. F. Pinaud, L. A. Bentolila, J. M. Tsay, S. Doose, J. J. Li, G. Sundaresan, A. Wu, S. Gambhir and S. Weiss, Science, 2005, 307, 538-544.

313 I. Gur, N. A. Fromer, M. L. Geier and A. P. Alivisatos, Science, 2005, 310, 462-465.

314 D. J. Norris, A. L. Efros and S. C. Erwin, Science, 2008, 319, 1776.

315 A. Hasani, Q. V. Le, T. P. Nguyen, K. S. Choi, W. Sohn, H. W. Jang and S. Y. Kim, Electrochim. Acta, 2018, 283, 1195-1202.

316 H. Gao, J. Suh, M. C. Cao, A. Y. Joe, F. Mujid, K.-H. Lee, S. Xie, P. Poddar, J.-U. Lee, K. Kang, P. Kim, D. A. Muller and J. Park, Nano Lett., 2020, 20, 4095-4101.

317 M. Kanehara, H. Koike, T. Yoshinaga and T. Teranishi, J. Am. Chem. Soc., 2009, 131, 17736-17737.

318 R. Buonsanti, A. Llordes, S. Aloni, B. A. Helms and D. J. Milliron, Nano Lett., 2011, 11, 4706-4710.

319 T. R. Gordon, T. Paik, D. R. Klein, G. V. Naik, H. Caglayan, A. Boltasseva and C. B. Murray, Nano Lett., 2013, 13, 2857-2863.

320 L. De Trizio, R. Buonsanti, A. M. Schimpf, A. Llordes, D. R. Gamelin, R. Simonutti and D. J. Milliron, Chem. Mater., 2013, 25, 3383-3390.

321 A. Hasani, Q. V. Le, M. Tekalgne, W. Guo, S. H. Hong, K. S. Choi, T. H. Lee, H. W. Jang and S. Y. Kim, ACS Appl. Mater. Interfaces, 2018, 10, 43785-43791.
322 J. Zhou, Y. Wei, G. Luo, J. Zheng and C. Xu, J. Mater. Chem. C, 2016, 4, 1613-1622.

323 G.-f. Cai, X.-l. Wang, D. Zhou, J.-h. Zhang, Q.-q. Xiong, C.-d. Gu and J.-p. Tu, RSC Adv., 2013, 3, 6896-6905.

324 H. Miyazaki, M. Inada, H. Suzuki and T. Ota, Bull. Chem. Soc. Jpn., 2012, 85, 1053-1056.

325 Y. Shen, P. Yan, Y. Yang, F. Hu, Y. Xiao, L. Pan and Z. Li, J. Alloys Compd., 2015, 629, 27-31.

326 C. O. Avellaneda, P. R. Bueno, R. C. Faria and L. O. S. Bulhões, Electrochim. Acta, 2001, 46, 1977-1981.

327 A. P. Alivisatos, Science, 1996, 271, 933-937.

328 M. Yao, Q. Li, G. Hou, C. Lu, B. Cheng, K. Wu, G. Xu, F. Yuan, F. Ding and Y. Chen, ACS Appl. Mater. Interfaces, 2015, 7, 2856-2866.

329 L. E. Brus, J. Chem. Phys., 1984, 80, 4403-4409.

330 A. Sahu, M. S. Kang, A. Kompch, C. Notthoff, A. W. Wills, D. Y. Deng, M. Winterer, C. D. Frisbie and D. J. Norris, Nano Lett., 2012, 12, 2587-2594.

331 A. W. Wills, M. S. Kang, K. M. Wentz, S. E. Hayes, A. Sahu, W. L. Gladfelter and D. J. Norris, J. Mater. Chem., 2012, 22, 6335-6342.

332 D. Chen, R. Viswanatha, G. L. Ong, R. Xie, M. Balasubramaninan and X. Peng, J. Am. Chem. Soc., 2009, 131, 9333-9339.

333 S. D. Lounis, E. L. Runnerstrom, A. Llordés and D. J. Milliron, J. Phys. Chem. Lett., 2014, 5, 1564-1574.

334 T. M. Mattox, A. Bergerud, A. Agrawal and D. J. Milliron, Chem. Mater., 2014, 26, 1779-1784.

335 D. Zhou, F. Shi, D. Xie, D. H. Wang, X. H. Xia, X. Wang, C. D. Gu and J. Tu, Bi-functional Mo-doped WO3 Nanowire Array Electrochromism-Plus Electrochemical Energy Storage, 2015.

336 N. M. Megahid, M. M. Wakkad, E. K. Shokr and N. M. Abass, Phys. B, 2004, 353, 150-163.

337 T. R. Gordon, T. Paik, D. R. Klein, G. V. Naik, H. Caglayan, A. Boltasseva and C. B. Murray, Nano Lett., 2013, 13, 2857-2863.

338 C. O. Avellaneda, P. R. Bueno and L. O. S. Bulhoes, J. NonCryst. Solids, 2001, 290, 115-121.

339 S. C. Erwin, L. Zu, M. I. Haftel, A. L. Efros, T. A. Kennedy and D. J. Norris, Nature, 2005, 436, 91-94.

340 D. Mocatta, G. Cohen, J. Schattner, O. Millo, E. Rabani and U. Banin, Science, 2011, 332, 77-81.

341 W. Liu, H. Bai, X. Li, W. Li, J. Zhai, J. Li and G. Xi, J. Phys. Chem. Lett., 2018, 9, 4096-4100.

342 S. Hsu, A. L. Rodarte, M. Som, G. Arya and A. R. Tao, Chem. Rev., 2018, 118, 3100-3120.

343 M. Wu, Y. Shi, R. Li and P. Wang, ACS Appl. Mater. Interfaces, 2018, 10, 39819-39827.

344 H. Zhu and L. Wang, Sol. Energy Mater. Sol. Cells, 2019, 202, 110109.

345 X. Xia, Z. Ku, D. Zhou, Y. Zhong, Y. Zhang, Y. Wang, M. J. Huang, J. Tu and H. J. Fan, Mater. Horiz., 2016, 3, 588-595.

346 J. Wang, J. Polleux, J. Lim and B. Dunn, J. Phys. Chem. C, 2007, 111, 14925-14931. 
347 E. L. Runnerstrom, A. Llordes, S. D. Lounis and D. J. Milliron, Chem. Commun., 2014, 50, 10555-10572.

348 X. Ye, J. Fei, B. T. Diroll, T. Paik and C. B. Murray, J. Am. Chem. Soc., 2014, 136, 11680-11686.

349 H. A. Atwater and A. Polman, Nat. Mater., 2010, 9, 205-213.

350 S. Zeb, X. Peng, Y. Shi, J. Su, J. Sun, M. Zhang, G. Sun, Y. Nie, Y. Cui and X. Jiang, Sens. Actuators, B, 2021, 334, 129584.

351 L. R. Hirsch, R. J. Stafford, J. A. Bankson, S. R. Sershen, B. Rivera, R. E. Price, J. D. Hazle, N. J. Halas and J. L. West, Proc. Natl. Acad. Sci. U. S. A., 2003, 100, 13549-13554.

352 C. Wang, X. Nie, Y. Shi, Y. Zhou, J. Xu, X. Xia and H. Chen, ACS Nano, 2017, 11, 5897-5905.

353 Y. Shi, J. Wang, C. Wang, T. Zhai, W. Bao, J. Xu, X. Xia and H. Chen, J. Am. Chem. Soc. Rev., 2015, 137, 7365-7370.

354 S.-J. Kim, S.-J. Choi, J.-S. Jang, H.-J. Cho and I.-D. Kim, Acc. Chem. Res., 2017, 50, 1587-1596.

355 R. M. Penner, Acc. Chem. Res., 2017, 50, 1902-1910.

356 G. Konvalina and H. Haick, Acc. Chem. Res., 2014, 47, 66-76.

357 I. H. Elsayed, X. Huang and M. A. Elsayed, Nano Lett., 2005, 5, 829-834.

358 U. Schurmann, H. Takele, V. Zaporojtchenko and F. Faupel, Thin Solid Films, 2006, 515, 801-804.

359 H. T. Beyene, V. S. K. Chakravadhanula, C. Hanisch, M. Elbahri, T. Strunskus, V. Zaporojtchenko, L. Kienle and F. Faupel, J. Mater. Sci., 2010, 45, 5865-5871.

360 S. Link and M. A. El-Sayed, J. Phys. Chem. B, 1999, 103, 8410-8426.

361 N. R. Jana, L. Gearheart and C. J. Murphy, J. Phys. Chem. B, 2001, 105, 4065-4067.

362 J. E. Millstone, S. Park, K. L. Shuford, L. Qin, G. C. Schatz and C. A. Mirkin, J. Am. Chem. Soc., 2005, 127, 5312-5313.

363 M. E. Mackay, A. Tuteja, P. M. Duxbury, C. J. Hawker, B. Van Horn, Z. Guan, G. Chen and R. S. Krishnan, Science, 2006, 311, 1740-1743.

364 M. E. Mackay, A. Tuteja, P. M. Duxbury, C. J. Hawker, B. A. Van Horn, Z. Guan, G. Chen and R. S. Krishnan, Science, 2006, 311, 1740-1743.

365 M. J. A. Hore, A. L. Frischknecht and R. J. Composto, ACS Macro Lett., 2012, 1, 115-121.

366 R. Buonsanti, T. E. Pick, N. Krins, T. J. Richardson, B. A. Helms and D. J. Milliron, Nano Lett., 2012, 12, 3872-3877.

367 R. Buonsanti, V. Grillo, E. Carlino, C. Giannini, T. Kipp, R. Cingolani and P. D. Cozzoli, J. Am. Chem. Soc., 2008, 130, 11223-11233.

368 J. Yan, S. Li, B. Lan, Y. Wu and P. S. Lee, Adv. Funct. Mater., 2020, 30, 1902564.

369 X. Wang, B. Liu, Q. Wang, W. Song, X. Hou, D. Chen, Y.-b. Cheng and G. Shen, Adv. Mater., 2013, 25, 1479-1486.

370 X. Wang, B. Liu, R. Liu, Q. Wang, X. Hou, D. Chen, R. Wang and G. Shen, Angew. Chem., Int. Ed., 2014, 53, 1849-1853.

371 C. Chen, J. Cao, Q. Lu, X. Wang, L. Song, Z. Niu and J. Chen, Adv. Funct. Mater., 2017, 27, 1604639.
372 R. Li, X. Ma, J. Li, J. Cao, H. Gao, T. Li, X. Zhang, L. Wang, Q. Zhang, G. Wang, C. Hou, Y. Li, T. Palacios, Y. Lin, H. Wang and X. Ling, Nat. Commun., 2021, 12, 1587.

373 J. Yun, Y. Lim, G. N. Jang, D. Kim, S.-J. Lee, H. Park, S. Y. Hong, G. Lee, G. Zi and J. S. Ha, Nano Energy, 2016, 19, 401-414.

374 D. Vonlanthen, P. Lazarev, K. A. See, F. Wudl and A. J. Heeger, Adv. Mater., 2014, 26, 5095-5100.

375 H. Kai, W. Suda, Y. Ogawa, K. Nagamine and M. Nishizawa, ACS Appl. Mater. Interfaces, 2017, 9, 19513-19518.

376 J. Zhi, M. Zhou, Z. Zhang, O. Reiser and F. Huang, Nat. Commun., 2021, 12, 445.

377 Z.-S. Wu, X. Feng and H.-M. Cheng, Natl. Sci. Rev., 2013, 1, 277-292.

378 C. Granqvist, Sol. Energy Mater. Sol. Cells, 2007, 91, 1529-1598.

379 K. Ellmer, Nat. Photonics, 2012, 6, 809-817.

380 L. Li, Z. Lou, D. Chen, K. Jiang, W. Han and G. Shen, Small, 2018, 14, 1702829.

381 X. Huang, Z. Zeng, Z. Fan, J. Liu and H. Zhang, Adv. Mater., 2012, 24, 5979-6004.

382 T. Q. Trung, L. T. Duy, S. Ramasundaram and N.-E. Lee, Nano Res., 2017, 10, 2021-2033.

383 T. Wang, Y. Guo, P. Wan, H. Zhang, X. Chen and X. Sun, Small, 2016, 12, 3748-3756.

384 H. C. Lee, W. Liu, S. Chai, A. R. Mohamed, C. W. Lai, C. Khe, C. H. Voon, U. Hashim and N. M. S. Hidayah, Procedia Chem., 2016, 19, 916-921.

385 K. S. Kim, Y. Zhao, H. Jang, S. Y. Lee, J. Kim, K. S. Kim, J. Ahn, P. Kim, J. Choi and B. H. Hong, Nature, 2009, 457, 706-710.

386 H. Peng, J. Am. Chem. Soc., 2008, 130, 42-43.

387 L. Hu, H. S. Kim, J. Lee, P. Peumans and Y. Cui, ACS Nano, 2010, 4, 2955-2963.

388 M. S. Miller, J. C. O. Kane, A. Niec, R. S. Carmichael and T. B. Carmichael, ACS Appl. Mater. Interfaces, 2013, 5, 10165-10172.

389 B. Xu and Y. Gogotsi, Adv. Funct. Mater., 2020, 30, 2007011. 390 E. Valkonen, B. Karlsson and C. Ribbing, Sol. Energy, 1984, 32, 211-222.

391 Z. Wang, X. Cai, Q. Chen and L. Li, Vacuum, 2006, 80, 438-443.

392 C. Yan, W. Kang, J. Wang, M. Cui, X. Wang, C. Y. Foo, K. J. Chee and P. S. Lee, ACS Nano, 2014, 8, 316-322.

393 M. F. Al-Kuhaili, A. H. Al-Aswad, S. M. A. Durrani and I. A. Bakhtiari, Sol. Energy, 2012, 86, 3183-3189.

394 V. Sharma, P. Kumar, A. Kumar, Surbhi, K. Asokan and K. Sachdev, Sol. Energy Mater. Sol. Cells, 2017, 169, 122-131.

395 M. Neghabi, A. Behjat, S. M. B. Ghorashi and S. M. A. Salehi, Thin Solid Films, 2011, 519, 5662-5666.

396 R. B. H. Tahar, T. Ban, Y. Ohya and Y. Takahashi, J. Appl. Phys., 1998, 83, 2631-2645.

397 J. Kim, S. Wong, G. Kim, Y.-B. Park, J. van Embden and E. Della Gaspera, J. Mater. Chem. C, 2020, 8, 14531-14539. 
398 H.-Y. Lu, C.-Y. Chou, J.-H. Wu, J.-J. Lin and G.-S. Liou, J. Mater. Chem. C, 2015, 3, 3629-3635.

399 H.-S. Liu, B.-C. Pan and G.-S. Liou, Nanoscale, 2017, 9, 2633-2639.

400 C. Lee, Y. Oh, I. S. Yoon, S. H. Kim, B.-K. Ju and J.-M. Hong, Sci. Rep., 2018, 8, 2763.

401 E. D. Martínez, C. D. S. Brites, L. D. Carlos, A. F. GarcíaFlores, R. R. Urbano and C. Rettori, Adv. Funct. Mater., 2019, 29, 1970045.

402 W.-H. Chen, F.-W. Li and G.-S. Liou, Adv. Opt. Mater., 2019, 7, 1900632.

403 H. Peng, X. Sun, F. Cai, X. Chen, Y. Zhu, G. Liao, D. Chen, Q. Li, Y. Lu, Y. Zhu and Q. Jia, Nat. Nanotechnol., 2009, 4, 738-741.

404 X. Chen, H. Lin, P. Chen, G. Guan, J. Deng and H. Peng, Adv. Mater., 2014, 26, 4444-4449.

405 W.-R. Lian, Y.-C. Huang, Y.-A. Liao, K.-L. Wang, L.-J. Li, C.Y. Su, D.-J. Liaw, K.-R. Lee and J.-Y. Lai, Macromolecules, 2011, 44, 9550-9555.

406 J. Palenzuela, A. Viñuales, I. Odriozola, G. Cabañero, H. J. Grande and V. Ruiz, ACS Appl. Mater. Interfaces, 2014, 6, 14562-14567.

407 L. Cui, X. Chen, B. Liu, K. Chen, Z. Chen, Y. Qi, H. Xie, F. Zhou, M. H. Rümmeli, Y. Zhang and Z. Liu, ACS Appl. Mater. Interfaces, 2018, 10, 32622-32630.

408 I. N. Kholmanov, S. H. Domingues, H. Chou, X. Wang, C. Tan, J.-Y. Kim, H. Li, R. Piner, A. J. G. Zarbin and R. S. Ruoff, ACS Nano, 2013, 7, 1811-1816.

409 K.-W. Kim, S. B. Lee, S. H. Kim and H. C. Moon, Org. Electron., 2018, 62, 151-156.

410 B. Che, D. Zhou, H. Li, C. He, E. Liu and X. Lu, Org. Electron., 2019, 66, 86-93.

411 K. Mallikarjuna and H. Kim, ACS Appl. Mater. Interfaces, 2019, 11, 1969-1978.

412 S. Abdolhosseinzadeh, X. Jiang, H. Zhang, J. Qiu and C. Zhang, Mater. Today, 2021, DOI: 10.1016/ j.mattod.2021.02.010.

413 Z. Fu, N. Wang, D. Legut, C. Si, Q. Zhang, S. Du, T. C. Germann, J. S. Francisco and R. Zhang, Chem. Rev., 2019, 119, 11980-12031.

414 C. Dulgerbaki and A. Uygun Oksuz, Polym. Adv. Technol., 2016, 27, 73-81.

415 H. Wang, M. Barrett, B. Duane, J. Gu and F. Zenhausern, Mater. Sci. Eng., B, 2018, 228, 167-174.

416 G. Zukowska, J. R. Stevens and K. R. Jeffrey, Electrochim. Acta, 2003, 48, 2157-2164.
417 C. G. Granqvist, Thin Solid Films, 2014, 564, 1-38.

418 G. Cai, J. Wang and P. S. Lee, Acc. Chem. Res., 2016, 49, 1469-1476.

419 M. Cui, W. S. Ng, X. Wang, P. Darmawan and P. S. Lee, Adv. Funct. Mater., 2015, 25, 401-408.

420 M. Cui and P. S. Lee, Chem. Mater., 2016, 28, 2934-2940.

421 T. Y. Yun, X. Li, J. Bae, S. H. Kim and H. C. Moon, Mater. Des., 2019, 162, 45-51.

422 C. M. Amb, A. L. Dyer and J. R. Reynolds, Chem. Mater., 2011, 23, 397-415.

423 W. Xu, J.-P. Belieres and C. A. Angell, Chem. Mater., 2001, 13, 575-580.

424 W. Xu, M. D. Williams and C. A. Angell, Chem. Mater., 2002, 14, 401-409.

425 C. A. Nguyen, A. A. Argun, P. T. Hammond, X. Lu and P. S. Lee, Chem. Mater., 2011, 23, 2142-2149.

426 D. Dong, B. Zhou, Y. Sun, H. Zhang, G. Zhong, Q. Dong, F. Fu, H. Qian, Z. Lin, D. Lu, Y. Shen, J. Wu, L. Chen and H. Chen, Nano Lett., 2019, 19, 2343-2349.

427 G. A. Niklasson and C. Granqvist, J. Mater. Chem., 2007, 17, 127-156.

428 D. Choi, M. Lee, H. Kim, W.-s. Chu, D.-m. Chun, S.-H. Ahn and C. S. Lee, Sol. Energy Mater. Sol. Cells, 2018, 174, 599-606.

429 K.-H. Kim, S.-J. Jeong, B.-R. Koo and H.-J. Ahn, Appl. Surf. Sci., 2021, 537, 147902.

430 S.-M. Wang, Y. Kim, B. Kim, M. Han and E. Kim, Adv. Funct. Mater., 2019, 29, 1806590.

431 Q. Guo, X. Zhao, Z. Li, B. Wang, D. Wang and G. Nie, ACS Appl. Energy Mater., 2020, 3, 2727-2736.

432 W. Guo, Z. Cong, Z. H. Guo, P. Zhang, Y. Chen, W. Hu, Z. L. Wang and X. Pu, Adv. Funct. Mater., 2021, 31, 2104348.

433 S. Fleischmann, J. B. Mitchell, R. Wang, C. Zhan, D.-e. Jiang, V. Presser and V. Augustyn, Chem. Rev., 2020, 120, 6738-6782.

434 W.-q. Wang, X.-l. Wang, X.-h. Xia, Z.-j. Yao, Y. Zhong and J.-p. Tu, Nanoscale, 2018, 10, 8162-8169.

435 R. Zheng, Y. Wang, J. Pan, H. A. Malik, H. Zhang, C. Jia, X. Weng, J. Xie and L. Deng, ACS Appl. Mater. Interfaces, 2020, 12, 27526-27536.

436 S. Y. Kim, T. Y. Yun, K. S. Yu and H. C. Moon, ACS Appl. Mater. Interfaces, 2020, 12, 51978-51986.

437 T. Y. Yun, X. Li, S. H. Kim and H. C. Moon, ACS Appl. Mater. Interfaces, 2018, 10, 43993-43999.

438 R. Wen, C. Granqvist and G. A. Niklasson, Adv. Funct. Mater., 2015, 25, 3359-3370. 